\title{
Monitoring and Evaluation of Supplemented Spring Chinook Salmon and Life Histories of Wild Summer Steelhead in the Grande Ronde Basin
}

\section{Annual Report}

\section{January 2007 through 31 December 2007}

\author{
Stephen J. Boe \\ Carrie A. Crump \\ Rey L. Weldert \\ Confederated Tribes of the Umatilla Indian Reservation \\ Department of Natural Resources, Fisheries Program \\ P. O. Box 638, Pendleton, Oregon 97801 \\ (541) 962-3043 \\ Prepared for: \\ Jonathan McCloud, Project Manager \\ U.S. Department of Energy \\ Bonneville Power Administration \\ Division of Fish and Wildlife \\ P. O. Box 3621 \\ Portland, Oregon 97208-3621 \\ Project 200708300 \\ BPA Contract No. 32603 \\ CTUIR Project 413
}

April 2009 
1 SECTION I. MONITORING AND EVALUATION OF ENDEMIC SPRING CHINOOK SALMON SUPPLEMENTATION IN CATHERINE CREEK AND THE UPPER GRANDE RONDE RIVER, OREGON ........................................................... 4

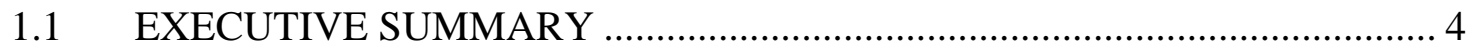

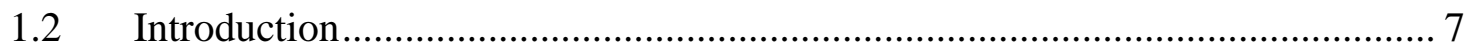

1.3 Study Area ........................................................................................... 8

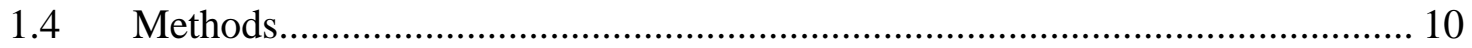

1.4.1 Juvenile Spring Chinook Salmon............................................................. 10

1.4.2 Adult Spring Chinook Salmon ................................................................. 12

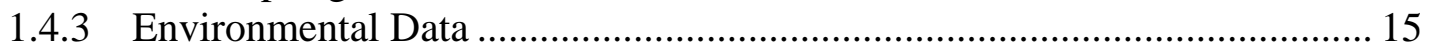

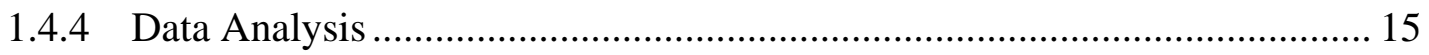

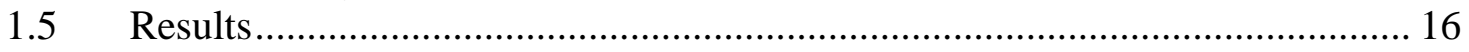

1.5.1 Catherine Creek Juvenile Spring Chinook Salmon ................................... 16

1.5.1.1 Size At Release ....................................................................... 16

1.5.1.2 Migration Timing.................................................................. 20

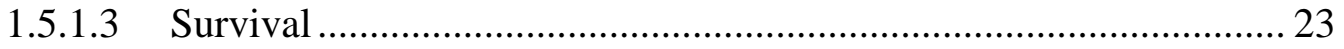

1.5.2 Catherine Creek Adult Spring Chinook Salmon ........................................ 23

1.5.2.1 Harvest .................................................................................... 23

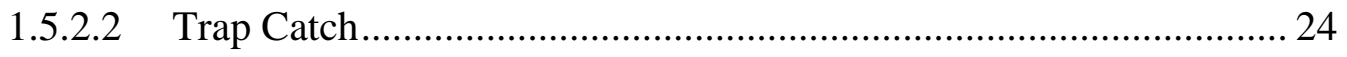

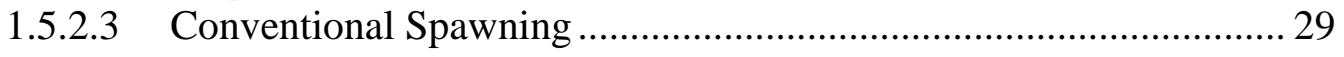

1.5.2.4 Spawning Ground Surveys ....................................................... 31

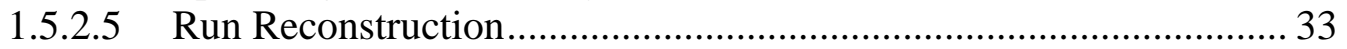

1.5.2.6 Run Size Predictors ................................................................. 37

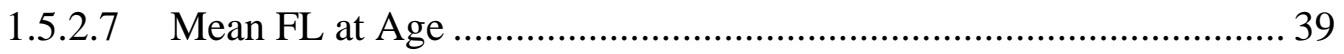

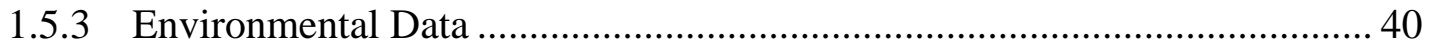

1.5.4 Upper Grande Ronde River Juvenile Chinook Salmon ............................. 42

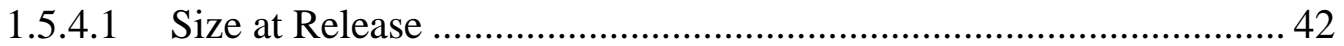

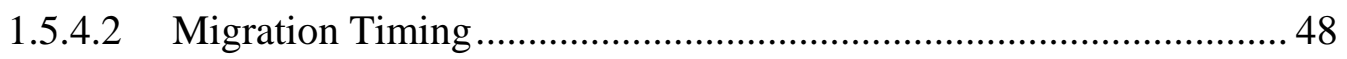

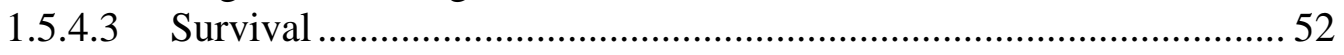

1.5.5 Upper Grande Ronde River Adult Spring Chinook Salmon........................ 53

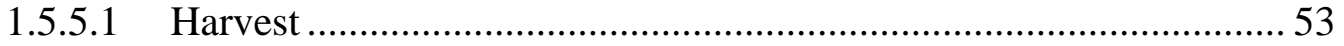

1.5.5.2 Trap Catch........................................................................ 54

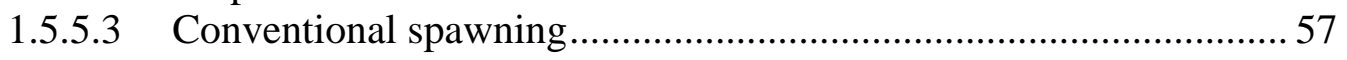

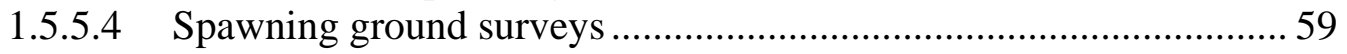

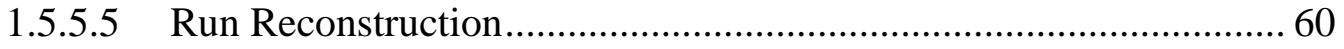

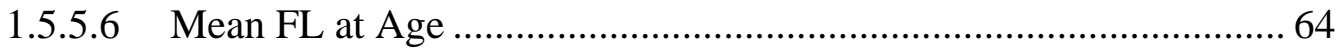

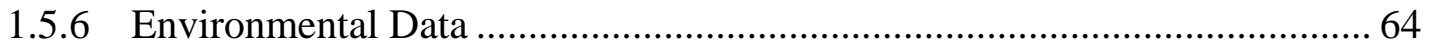

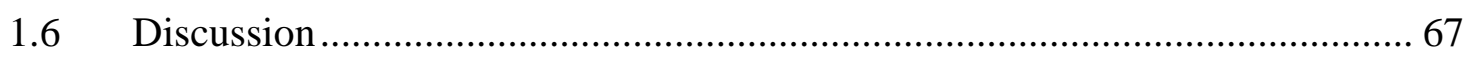

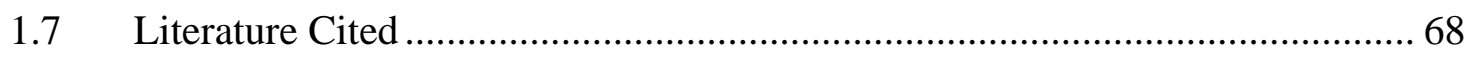

2 SECTION II. SUMMER STEELHEAD IN CATHERINE CREEK AND THE

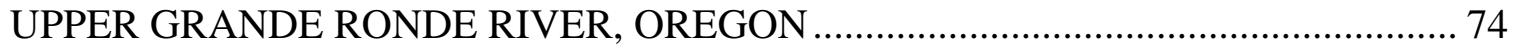




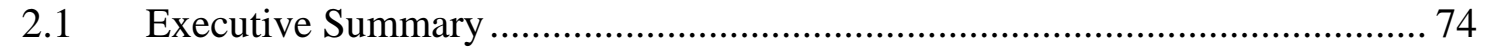

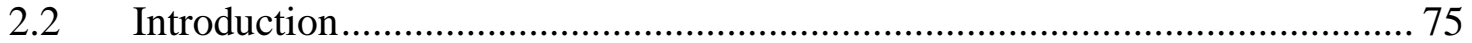

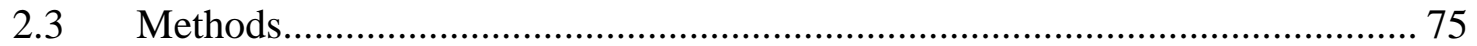

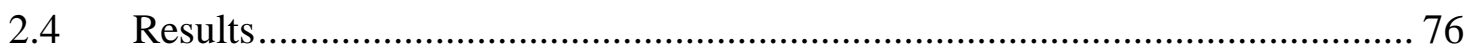

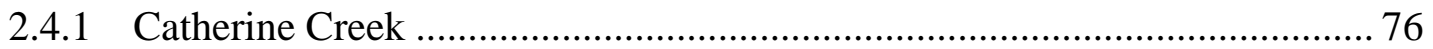

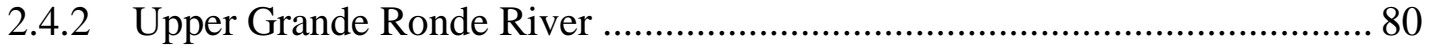

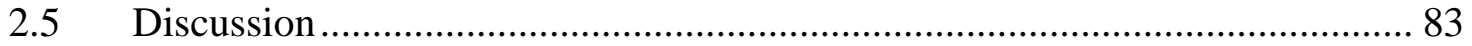

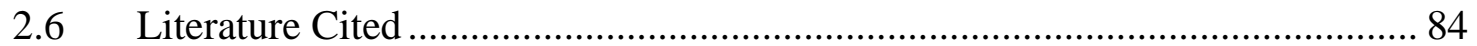

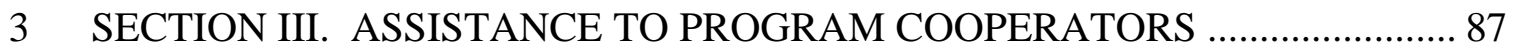

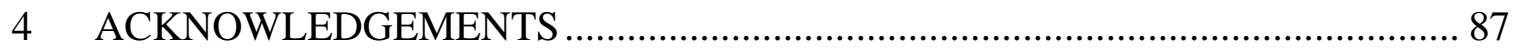




\section{SECTION I. MONITORING AND EVALUATION OF ENDEMIC SPRING CHINOOK SALMON SUPPLEMENTATION IN CATHERINE CREEK AND THE UPPER GRANDE RONDE RIVER, OREGON}

\subsection{EXECUTIVE SUMMARY}

This is the ninth annual report for a multi-year project designed to monitor and evaluate supplementation of endemic spring Chinook salmon in Catherine Creek and the upper Grande Ronde River. These two streams historically supported anadromous fish populations that provided significant tribal and non-tribal fisheries, but in recent years, have experienced severe declines in abundance. Conventional and captive broodstock supplementation methods are being used to restore these spring Chinook salmon populations.

Statement of Work Objectives for 2007 included:

1. Participate in management forums and develop management, monitoring and evaluation strategies and master plans in cooperation with co-managers for the restoration of salmon, steelhead and bull trout and their habitats within the Grande Ronde River Basin.

2. Evaluate and contrast performance of supplemented endemic juvenile spring Chinook salmon of conventional and captive broodstock hatchery origin acclimated and released at facilities on Catherine Creek and the upper Grande Ronde River and naturallyproduced juveniles.

3. Evaluate life history differences between natural and supplemented (hatchery-origin $F_{1}$ ) adult spring Chinook salmon from Catherine Creek and the upper Grande Ronde River.

4. Describe life history characteristics and genetic stock structure of adult summer steelhead from Catherine Creek and the upper Grande Ronde River.

5. Assist comanagers with program activities.

This project included operations and maintenance (O\&M) and monitoring and evaluation (M\&E) components prior to 2002. Beginning in 2002, project O\&M and M\&E responsibilities were split into different contracts and CTUIR projects (M\&E, 413 and O\&M, 410), and separate budget and reporting requirements, but with the same Bonneville Power Administration (BPA) Project \# of 1998-007-03. Beginning in 2007, M\&E responsibilities received a separate BPA Project \# of 2007-083-00. 
A total of 71,268 brood year (BY) 2005 Catherine Creek spring Chinook salmon juveniles were acclimated and released in one group from 12 March 2007-11 April 2007 (forceout). Volitional release began on 26 March 2007. A total of 139,423 upper Grande Ronde River juveniles were acclimated and released in two groups from 12 March-11 April 2007. The first group was acclimated from 12 March-25 March (forceout), with volitional release starting 19 March. The second group was acclimated from 26 March11 April (forceout), with volitional release starting 2 April. All juveniles were marked with one or more of the following: coded-wire tags (CWT), adipose fin clips, and PIT tags. Approximately 2\% of the upper Grande Ronde River and 29\% of the Catherine Creek juveniles were PIT-tagged. Catherine Creek captive broodstock progeny received visual implant elastomer (VIE) tags in the fatty tissue posterior to the eye.

Mean FL (mm) at PIT-tagging in October 2006 for various groups of Catherine Creek hatchery-origin fish released in March-April 2007 ranged from 98.0-107.4 (overall mean 102.3), compared to 90.3 for natural-origin. Median arrival dates at Lower Granite Dam for various groups of hatchery-origin fish from ranged from 3 May-11 May 2007 (overall median 10 May 2007). The median for natural-origin juveniles was 13 May 2007. Harmonic mean travel times ranged from 38.872-45.590 d (overall mean 43.229) for hatchery-origin fish compared to $39.125 \mathrm{~d}$ for natural-origin fish. Survival rates for hatchery-origin fish ranged from 0.3245-0.3828 (overall 0.3249), compared to 0.3111 for natural-origin fish.

Adult spring Chinook salmon collected at the Catherine Creek trap totaled 242 (115 females, 127 males). Natural-origin adults caught from 14 May-25 June totaled 77 (36 females, 41 males) and hatchery-origin adults caught from 21 May-25 June totaled 165 (79 females, 86 males). Slightly more natural-origin adults arrived at the trap earlier than hatchery-origin. Conventional broodstock spawned at Lookingglass Hatchery totaled 28 natural- (14 of each sex) and 47 hatchery-origin (31 females, 16 males). Peak spawning for both hatchery- and natural-origin females occurred on 29 August. Mean fecundity of hatchery-origin females was 3,924 compared to 3,726 for natural-origin. Mean egg weights were $0.23 \mathrm{~g}$ (natural-origin) and $0.24 \mathrm{~g}$ (hatchery-origin).

A total of 57 redds were observed above the Catherine Creek trap and 2 below. Total carcass recoveries were 21 above the trap and 1 below. Estimated total returns to the stream for hatchery-origin ages 3, 4, and 5 were 29, 146, and 6, respectively. Estimated hatchery-origin instream spawners for ages 3, 4, and 5 were 2, 109, and 6, respectively. Estimated total returns to the stream for natural-origin ages 3, 4, and 5 were 7, 42, and 35, respectively. Estimated natural-origin instream spawners ages 3, 4, and 5 were 6, 29, and 20, respectively. Total returns of brood year 2002 hatchery-origin releases for run years 2005-2006-2007 were 246 and total instream spawners were 180. Total returns of brood year 2002 natural-origin outmigrants for run years 2005-2006-2007 were 178 and total 
instream spawners were 124. Smolt-to-adult ratios for brood year 2002 were $0.15 \%$ for hatchery-origin and 5.40\% for natural-origin. Progeny-per-parent ratio for the 2002 brood year was 0.36 .

Regressing age 3 natural-origin returns on age 4 returns failed to produce a significant relationship. A significant relationship was obtained by regressing age 3 hatchery-origin returns on age 4 returns if an outlier was eliminated.

Mean FL at age 3 was not significantly different between hatchery- and natural-origin returns. Mean FL at age 4 was significantly different between hatchery- and naturalorigin males and females.

Mean FL at PIT-tagging for various groups of hatchery-origin upper Grande Ronde River fish ranged from 105.3-115.3 mm (overall mean 106.4), compared to 82.5-85.0 mm for two groups of natural-origin outmigrants (overall mean $83.8 \mathrm{~mm}$ ). Median arrival date at Lower Granite Dam for hatchery-origin upper Grande Ronde River fish ranged from 1 May-5 May 2007 (overall median 3 May 2007), compared to 13 -18 May 2007 for two groups of natural-origin outmigrants (overall median 14 May 2007). Harmonic mean travel times ranged from 29.725-46.149 d (overall mean $33.166 \mathrm{~d}$ ) for various groups of hatchery-origin and 39.031-40.626 d for two groups of natural-origin outmigrants (overall mean 42.216). Survival rates ranged from 0.3270-0.5602 (overall 0.4956) for various groups of hatchery-origin and 0.2109-0.3469 for two groups of natural-origin outmigrants (overall 0.3172).

Adult spring Chinook salmon collected at the upper Grande Ronde River trap totaled 73 39 females, 34 males). Natural-origin adults caught from 21 May-27 June totaled 33 (17 females, 16 males) and hatchery-origin adults caught from 21 May-25 June totaled 40 (22 females, 18 males). Arrival timing at the trap was similar for hatchery- and natural-origin adults. Conventional broodstock spawned at Lookingglass Hatchery totaled 14 naturalorigin (6 females, 8 males) and 46 hatchery-origin ( 26 females, 20 males). Equal numbers of natural-origin females (2) were spawned on 6, 11 and 17 September. Peak spawning for hatchery-origin females occurred on 11 September (13). Mean fecundity of hatchery-origin females was 3,963 compared to 3,946 for natural-origin. Median egg weights were $0.22 \mathrm{~g}$ (natural-origin) and $0.23 \mathrm{~g}$ (hatchery-origin).

A single redd was observed above the upper Grande Ronde River trap and none below. A single carcass was also recovered above the trap. Estimated total returns to the stream for hatchery-origin ages 3, 4, and 5 were 18, 28, and 9 respectively. Estimated hatcheryorigin instream spawners ages 3, 4, and 5 were 3, 0, and 0, respectively. Estimated total returns to the stream for natural-origin ages 3,4 , and 5 were 0,21 , and 15 respectively. Estimated natural-origin instream spawners ages 3,4 , and 5 were 0,8 , and 8 , 
respectively. Total returns of brood year 2002 hatchery-origin for run years 2005-20062007 were 166 and total instream spawners were 33. Total returns of brood year 2002 natural-origin outmigrants for run years 2005-2006-2007 were 67 and total instream spawners were 34. Smolt-to-adult ratios for brood year 2002 were $0.11 \%$ for hatcheryorigin and $0.56 \%$ for natural-origin.

There were no significant differences by origin (hatchery or natural) in mean FL at age for age 4 females, age 5 males, or age 5 females. There was a significant difference in mean FL at age between age 4 hatchery- and natural-origin males.

Differences in spring Chinook salmon life history between hatchery- and natural-origin outmigrants were observed for both streams. Differences in juvenile life history result in large measure from rearing and release practices. Differences in adult life history between hatchery- and natural-origin adults were less common, and most noticeable in length-at-age and age structure.

Program assistance provided to comanagers in 2007 included collection of captive broodstock parr from Catherine Creek and the upper Grande Ronde River, maturity sorts and spawning activities at Bonneville Hatchery and Lookingglass Hatchery and spawning ground surveys in the Grande Ronde, Imnaha, and Wenaha basins.

\section{$1.2 \quad$ Introduction}

Spring Chinook salmon populations in Catherine Creek and the upper Grande Ronde River, and other streams in the Snake River Basin have experienced severe declines in abundance over the past two decades (Nehlsen et al. 1991). A supplementation program was initiated in Catherine Creek and the upper Grande Ronde River, incorporating the use of both captive and conventional broodstock methods, in order to prevent extinction in the short term and eventually rebuild populations. The captive broodstock component of the program (BPA Project 199801001) uses natural-origin parr collected by seining and reared to maturity at facilities near Seattle, Washington (Manchester Marine Laboratory) and Hood River, Oregon (Bonneville Hatchery). Spawning occurs at Bonneville Hatchery, and resulting progeny are reared in hatcheries. Shortly before outmigration in the spring, juveniles are transferred to acclimation facilities. After an acclimation period of about 2-4 weeks, volitional release begins. Any juveniles remaining after the volitional release period are forced out. The conventional broodstock component uses returning adults collected at traps near the spawning areas, transported to Lookingglass Hatchery near Elgin, Oregon, held, and later spawned. The resulting progeny are reared, acclimated, and released similar to the captive broodstock component. 
All progeny released receive one or more marks including a fin (adipose) clip, codedwire tag, PIT tag, or visual implant elastomer tag. The numbers of adults used for conventional broodstock are determined by an agreement among comanagers (Zimmerman and Patterson 2002). Activities for this project focus on two life stages of spring Chinook salmon: juveniles during the migration from freshwater to the ocean and adults during prespawning migration through the end of spawning. Life history, production, and genetics are monitored and used to evaluate program effectiveness.

\subsection{Study Area}

The Grande Ronde River originates in the Blue Mountains of northeastern Oregon and flows for $341 \mathrm{~km}$ to join the Snake River near Lewiston, Idaho (Figure 1). Gradient is moderately steep in the upper reaches, becoming more gradual from La Grande, Oregon, to the mouth. The Grande Ronde River drains a sparsely populated watershed of approximately $13,727 \mathrm{~km}^{2}$ dominated by agriculture, logging and outdoor recreation. The largest cities are La Grande (population 12,000), Enterprise (1,905), Union $(1,880)$, Elgin $(1,600)$, and Joseph $(1,054)$. Land ownership in the watershed is $53 \%$ private, $46 \%$ U. S. Forest Service, and less than $1 \%$ each by the U. S. Bureau of Land Management, and state and tribal agencies. Detailed information about the upper Grande Ronde River basin is contained in the Grande Ronde Subbasin Plan available at http://www.nwcouncil.org/fw/subbasinplanninng/granderonde/plan/. 


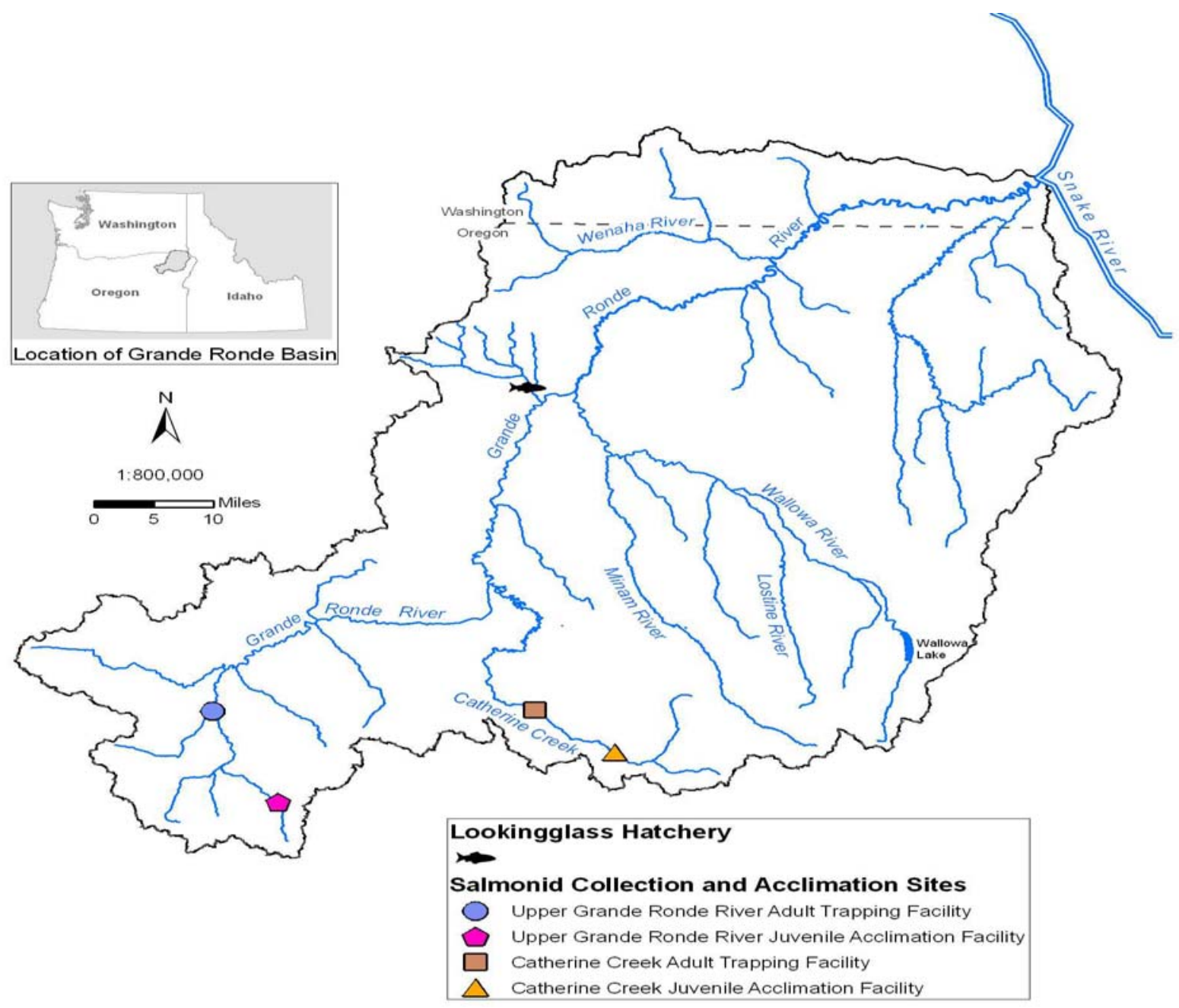

Figure 1. The Grande Ronde Basin, showing Lookingglass Hatchery, adult collection traps, and juvenile acclimation sites. 


\section{$1.4 \quad$ Methods}

\subsubsection{Juvenile Spring Chinook Salmon}

ODFW staff marked juvenile spring Chinook salmon at Lookingglass Hatchery in the summer of 2006 with adipose clips and coded wire tags. All Catherine Creek juveniles received adipose fin (ad) clips and coded wire tags (CWT). Captive broodstock progeny also received VIE (visual implant elastomer) tags. Upper Grande Ronde River conventional broodstock progeny received CWT but were not ad-clipped. Captive broodstock progeny received ad clips and CWT.

ODFW staff assisted by CTUIR and Nez Perce Tribe (NPT) PIT-tagged a portion of the Catherine Creek and upper Grande Ronde River juveniles at Lookingglass Hatchery in October 2006. A total of 1,985 from the upper Grande Ronde River were PIT -tagged, in keeping with the 500 per raceway standard for this stock. A total of 20,817 from Catherine Creek were PIT-tagged as part of the Comparative Survival Study (Bouwes et al. 2002).

FL (mm) and weight $(0.1 \mathrm{~g})$ data were collected from PIT-tagged fish at the time of tagging. ODFW, assisted by CTUIR and NPT, completed length, weight, and mark retention sampling at Lookingglass Hatchery prior to transfer to the acclimation facilities on 7-8 February 2007 (ODFW, unpublished data). Juveniles were also sampled at the Catherine Creek acclimation facility (50 fish per raceway) on 23 March 2007 (before volitional release of early group), 25 March 2007 (before forceout of early group), and 12 April 2007 (before volitional release of late group). Acclimation and release procedures were described in McLean et al. (2007). Juveniles were acclimated in two groups ("early" and "late”) at the upper Grande Ronde facility due to space constraints (Table 1).

Performance metrics monitored for hatchery-origin spring Chinook salmon juveniles included migration timing (median arrival timing to Lower Granite Dam), mean travel time to Lower Granite Dam, mean FL, mean weight, mean $\mathrm{K}$ factor $\left(\mathrm{K}=\mathrm{W} / \mathrm{FL}^{3} * 10^{5}\right.$, where $\mathrm{W}=$ weight in grams and $\mathrm{FL}=$ fork length in $\mathrm{mm}$ ), survival to Lower Granite Dam, and smolt-to-adult ratio (SAR). 
Table 1. Acclimation summary for brood year 2005 juvenile spring Chinook salmon from Catherine Creek (CC) and the upper Grande Ronde River (UGR), 2007 (data from McLean et al. 2007).

\begin{tabular}{cccccc}
\hline Stream & Period & $\begin{array}{c}\text { Broodstock } \\
\text { Method }\end{array}$ & $\begin{array}{c}\text { Volitional } \\
\text { Release }\end{array}$ & Forceout & $\begin{array}{c}\text { Number } \\
\text { Released }\end{array}$ \\
\hline CC & $*$ & Captive & 26 March 2007 & 11 April 2007 & 21,572 \\
CC & $*$ & Conventional & 26 March 2007 & 11 April 2007 & 49,696 \\
UGR & Early & Captive & 19 March 2007 & 25 March 2007 & 20,620 \\
UGR & Late & Conventional & 2 April 2007 & 11 April 2007 & 118,803 \\
\hline
\end{tabular}

* only one acclimation period in 2007

Date and time-stamped detections of PIT-tagged juveniles leaving each acclimation facility were recorded with PIT tag readers (Digital Angel Model FS2001 ${ }^{1}$ ) placed on outlet pipes at each facility. Placement of the PIT tag readers at the Catherine Creek facility potentially allowed detection of all juveniles leaving the facility. Readers probably detected a high percentage (assumed to be over 90\%) of those volitionally released. The large number of PIT-tagged fish leaving this facility in a short period of time at force-out meant that a significant number of tags were not detected due to tag interference (multiple tags passing through the field at essentially the same time). We assumed that PIT-tagged fish detected at release were representative of the particular group of PIT-tagged fish for the metrics used.

PIT tag data from the web-based PTAGIS database (Bauman et al. 1998) were used to describe migration timing and survival to Lower Granite Dam. To describe arrival timing at Lower Granite Dam, daily PIT tag detections were expanded for spill using flow data from the U. S. Army Corps of Engineers, Portland District website at http://www.nwdwc.usace.army.mil/perl/dataquery.pl?k=id:LWG, and calculating a daily expansion factor [(Powerhouse Outflow + Spill) /Powerhouse Outflow]. Median arrival timing at Lower Granite Dam for each group was the date by which $50 \%$ of the expanded detections had occurred. Survival, capture probabilities, and travel time to Lower Granite Dam were calculated using PitPro (Westhagen and Skalski 2006). We used the standard configuration, and excluded the *.rcp file. Observation sites used, in downstream order, were Lower Granite Dam, Little Goose Dam, Lower Monumental Dam, McNary Dam, John Day Dam, Bonneville Dam, and the Estuary Towed Array (Juvenile). Lower Granite Dam was used as the last recapture site. Natural-origin spring Chinook juveniles from both Catherine Creek and the upper Grande Ronde River used for performance comparisons with hatchery-origin fish were collected in rotary screw traps and PITtagged by ODFW (Jonasson et al. 2002). We used natural-origin juveniles PIT-tagged

\footnotetext{
${ }^{1}$ Mention of this commercial product does not imply endorsement.
} 
and released during the same time periods as hatchery-origin juveniles were released in comparison.

\subsubsection{Adult Spring Chinook Salmon}

Adult spring Chinook salmon life history attributes described for Catherine Creek and upper Grande Ronde River stocks included migration timing (arrival at traps), smolt-toadult ratios (SAR), progeny-per parent ratios, abundance, age, length and sex frequencies, hatchery/natural ratios, occurrence of strays, spawn timing, redd distribution, prespawning mortality, and egg size.

Most of the adult life history information for this project was collected at adult traps, during spawning ground surveys, and at Lookingglass Hatchery when adults are spawned. There were no tribal or sport fisheries for Catherine Creek or the upper Grande Ronde River in 2007. ODFW, assisted by comanagers CTUIR and NPT and others, coordinates and conducts spawning ground surveys of known spawning areas of Catherine Creek and the upper Grande Ronde River during 3 consecutive weeks in late August and early September (Parker et al. 1995). Data are also collected during additional pre- and post-spawn surveys and spawning of conventional broodstock at Lookingglass Hatchery. McLean et al. (2007) described trapping operations and methods used to collect data for spring Chinook salmon captured at the upper Grand Ronde River and Catherine Creek adult traps. Rarely, fish that had been previously trapped and released upstream fell back over the weir, swam upstream and were trapped again; these fish were omitted from the data summaries. Some fish from each stream were removed to maintain appropriate hatchery/natural ratios and some were taken to Lookingglass Hatchery for use as broodstock. Only unmarked (natural-origin) spring Chinook salmon or conventional broodstock progeny are currently used for conventional broodstock. Scales were used to age natural-origin spring Chinook salmon (Mosher 1969) and coded wire tags for hatchery-origin fish. Coded wire tag data were obtained from the database maintained by the Pacific States Marine Fisheries Commission at http://www.rmpc.org.

Spawners above the traps were estimated using the Chapman modification of the Petersen method of mark and recapture (Ricker 1975). All adults released above the trap were marked with an opercle punch. The recapture sample consisted of recoveries on spawning ground surveys (ODFW, unpublished data). The expanded numbers of spawners above the traps were estimated using population estimates, the fractions of hatchery- and natural-origin adults in the trap catch and those released above the trap, and age composition. Age compositions of hatchery- and natural-origin adults were based on age-length data from coded wire tag recoveries and scales.

Spawners below the traps were estimated from the product of redds and 2.5 fish/redd (Park 1990). Spawners below the traps were apportioned between hatchery- and natural- 
origin based mark status of the catch at the trap. Age composition of hatchery- and natural-origin spawners was estimated the same as for spawners above the trap.

Total returns to the stream were the sums of spawners above and below the traps, trap mortalities, those transported to Lookingglass Hatchery for use as conventional broodstock, those euthanized to maintain desired age 3:ages 4/5 and hatchery:natural ratios, and outplants (none in 2007). Total instream spawners was the sum of spawners above and below the trap.

Smolt-to-adult ratios were estimated using the number of hatchery-origin adult returns at ages 3-4-5 divided by the number of hatchery-origin smolts released and the number of natural-origin adult returns at ages 3-4-5 divided by the number of smolt equivalents to Lower Granite Dam (Brian Jonasson, personal communication). Progeny-per-parent ratios were estimated by dividing the number of age 4-5 instream spawners in a brood year by the sum of the number of natural-origin returns 4 and 5 years later.

Spring Chinook salmon experience low mortality from ocean fisheries before beginning their return to natal streams. Harvest occurs in the Columbia River from both non-tribal and tribal fisheries. Non-tribal fisheries include non-selective gillnet fisheries and selective (ad-clipped harvest only) sport fisheries. Tribal fisheries include non-selective ceremonial and subsistence fisheries using platform and set nets, and selective fisheries using hook and line. Estimated totals harvested in both non-trial and tribal fisheries are summarized by ODFW at http://www.dfw.state.or.us/fish/OSCRP/CRM/reports.asp. If adequate numbers of hatchery-origin adults return, sport or tribal fisheries may be opened in some Grande Ronde River tributaries; surveys are conducted to monitor catch and harvest. CWT information from harvested adults was obtained from the RMIS website maintained by PSMFC. There were no CWT data available for run year 2007 at the writing of this report.

Columbia River harvest of returning BY 2002 adult spring Chinook salmon occurred during 2005-2007. Total harvest annually by non-tribal fisheries ranged from 6,0129,987 and tribal fisheries from 6,144-8,401 (Table 2). Sport fisheries harvested 23-26\% of the non-tribal harvest for 2005-2007. Tribal harvest was essentially all for ceremonial and subsistence use during 2005-2007; there were no commercial fisheries. 
Table 2. Estimated harvest of spring Chinook salmon in the Columbia River, 2005-2007.

\begin{tabular}{|c|c|c|c|}
\hline Year & Group & Fishery & Harvest \\
\hline 2005 & Nontribal & Commercial & 2,246 \\
\hline 2005 & Nontribal & Sport & 7,684 \\
\hline \multirow[t]{2}{*}{2005} & Nontribal & Misc & 57 \\
\hline & & Total & 9,987 \\
\hline 2005 & Tribal & Winter Gillnet & 1 \\
\hline \multirow[t]{2}{*}{2005} & Tribal & $C \& S^{a}$ & 6,163 \\
\hline & & Total & 6,164 \\
\hline 2006 & Nontribal & Commercial & 1,689 \\
\hline 2006 & Nontribal & Sport & 4,809 \\
\hline \multirow[t]{2}{*}{2006} & Nontribal & Misc & 130 \\
\hline & & Total & 6,628 \\
\hline 2006 & Tribal & Winter Gillnet & 0 \\
\hline \multirow[t]{2}{*}{2006} & Tribal & C\&S & 8,401 \\
\hline & & Total & 8,401 \\
\hline 2007 & Nontribal & Commercial & 1,430 \\
\hline 2007 & Nontribal & Sport & 4,529 \\
\hline \multirow[t]{2}{*}{2007} & Nontribal & Misc & 53 \\
\hline & & Total & 6,012 \\
\hline 2007 & Tribal & Winter Gillnet & 3 \\
\hline \multirow[t]{2}{*}{2007} & Tribal & C\&S & 6,141 \\
\hline & & Total & 6,144 \\
\hline
\end{tabular}

a ceremonial and subsistence

Total escapement (hatchery-, natural-, and wild-origin) past Lower Granite Dam during 2005-2007 ranged from 22,481-26,027 ages 4/5 and 981-8,971 age 3

(http://www.cbr.washington.edu/dart/dart.html).

Different marking options are used for hatchery-origin juvenile spring Chinook salmon released into Catherine Creek and the upper Grande Ronde River. All Catherine Creek releases receive internal (CWT) and external (adipose clip and/or VIE tag) mark. These adults are subject to harvest in the non-selective and selective fisheries. Some upper Grande Ronde River juveniles are marked like Catherine Creek stock (with both internal and external marks) and some are marked with just an internal mark (CWT). As 
returning adults, those without external marks are treated as "wild" fish in ad-selective fisheries (i.e. sport), reducing harvest mortality and providing for more adult returns to the spawning grounds. Genetics tissues were collected from all adults collected at the traps and any carcasses observed on spawning ground surveys without opercle punches. Samples were preserved in labeled vials with about $2 \mathrm{ml}$ of $95 \%$ ethanol and later sent to the NOAA-Fisheries genetics laboratory as part of BPA Project 198909600. A second set of samples was sent to the Columbia River Inter-Tribal Fish Commission (CRITFC) genetics laboratory for archiving.

\subsubsection{Environmental Data}

The Wallowa-Whitman National Forest (water temperature) and Oregon Water Resources Department (stream flow) operate data recorders on the North Fork of Catherine Creek about 4.4 rkm above the acclimation site and on the upper Grande Ronde River below the mouth of Clear Creek at the old Woodley Campground, about 3.2 rkm above the upper Grande Ronde River acclimation site. Methods used for calculations of instantaneous flows and summary statistics are available at the Oregon Water Resources website (http://www.wrd.state.or.us/surface_water/data.shtml). Stream flow data were not available at the time this report was prepared. Water temperature data were also recorded hourly at the Catherine Creek acclimation and Catherine Creek and upper Grande Ronde River adult trap sites using Onset ${ }^{2}$ programmable data loggers (McLean et al. 2007). The 7-day maximum average daily water temperature was estimated by subtracting $1^{\circ} \mathrm{C}$ from the maximum daily water temperature. Flow data for the Grande Ronde River near Troy, OR were obtained from the U. S. Geological Service website (http://waterdata.usgs.gov/or/nwis/dv/?site_no=13333000\&amp;referred_module=sw), Water temperature and river flow at Lower Granite Dam were obtained from https://www.nwd-

wc.usace.army.mil/perl/dataquery.pl?k=id:LWG+record://LWG/TW//IRMONTH/IRGZZAZD/+psy:+psm:+psd:+pey:+pem:+ped:+pk:lower+granite+dam.

\subsubsection{Data Analysis}

Graphs and descriptive statistics in tables were used to summarize data for different life stages and groups of spring Chinook salmon. Least squares regression using $\log _{10}$ transformed data was used to describe length-weight relationships for female conventional broodstock spawned at Lookingglass Hatchery. Least squares regression was also used to predict abundance of age 4 returns from age 3 returns for Catherine Creek. Group differences in mean FL and K for juvenile and adult spring Chinook

\footnotetext{
${ }^{2}$ Mention of this commercial product does not imply endorsement.
} 
salmon were evaluated using t-tests. Group differences in arrival timing for juveniles were evaluated using the Wilcoxon test. Group differences in arrival timing and spawn timing for adults were tested using the Kolmogorov-Smirnov test. All statistical tests except for Kolmogorov-Smirnov were carried out using JMP 6 for Windows JMP 6 (SAS Institute, Inc. 2006). The Kolmogorov-Smirnov test was carried out using Statistix 7 (Analytical Software 2000). Tests were considered significant if $\mathrm{P}<0.05$.

\subsection{Results}

\subsubsection{Catherine Creek Juvenile Spring Chinook Salmon}

\subsubsection{Size At Release}

There were significant differences in mean FL at PIT-tagging between captive and conventional broodstock progeny, volitional release and forceout and between the entire groups of hatchery-origin and natural origin outmigrants (Table 3). Captive broodstock progeny were larger than conventional broodstock, volitionally-released were larger than forceout, and hatchery-origin juveniles were larger than natural.

Table 3. FL at PIT-tagging for brood year (BY) 2005 spring Chinook salmon by rearing and release type from Catherine Creek (CTUIR data and ODFW, unpublished data).

\begin{tabular}{cccccccc}
\hline & & \multicolumn{7}{c}{ FL $(\mathrm{mm})$} \\
\cline { 3 - 8 } Rearing $^{\mathrm{a}}$ & Release $^{\mathrm{b}}$ & Mean & SE & Range & $\mathrm{N}$ & $t$ ratio & $\mathrm{P}>|\mathrm{t}|$ \\
\hline $\mathrm{H}$ & CaV & 107.4 & 0.5 & $83-153$ & 358 & 3.615885 & 0.0003 \\
$\mathrm{H}$ & CaFO & 104.5 & 0.5 & $82-131$ & 250 & & \\
& & & & & & & \\
$\mathrm{H}$ & $\mathrm{CoV}$ & 100.6 & 0.2 & $80-117$ & 590 & 6.188492 & $<0.0001$ \\
$\mathrm{H}$ & CoFO & 98.0 & 0.3 & $75-116$ & 396 & & \\
& & & & & & & \\
$\mathrm{H}$ & $\mathrm{Ca}$ & 105.6 & 0.2 & $75-156$ & 1,576 & -23.4729 & $<0.0001$ \\
$\mathrm{H}$ & $\mathrm{Co}$ & 99.0 & 0.2 & $58-117$ & 1,566 & & \\
& & & & & & & \\
$\mathrm{H}$ & All & 102.3 & 0.2 & $58-156$ & 3,142 & & \\
$\mathrm{~N}$ & All & 90.3 & 0.5 & $75-110$ & 139 & & \\
\hline
\end{tabular}

${ }^{\mathrm{a}} \mathrm{H}=$ hatchery, $N=$ natural-origin tagged/released 26 March 2007-11 May 2007, ${ }^{\mathrm{b}}$ Ca $=$ captive broodstock progeny, $C o=$ conventional broodstock progeny, $V=$ volitional release detection, $F O=$ forceout detections

Mean FL increases between PIT-tagging in October 2006 and pretransfer sampling in February 2007 increased $3.8 \mathrm{~mm}$ for captive broodstock progeny and $11.5 \mathrm{~mm}$ for conventional broodstock progeny (Table 4). Mean FL of all hatchery-origin juveniles 
sampled at PIT-tagging and at pretransfer sampling increased $7.6 \mathrm{~mm}$. None of the between group hatchery-origin comparisons at pretransfer or acclimation facility sampling were significantly different. Mean FL at prevolitional or preforceout sampling at the acclimation facilities ranged from $6.2-10.5 \mathrm{~mm}$ greater than at pretransfer sampling in February 2007. Volitionally-released juveniles were slightly larger $(0.7-1.0 \mathrm{~mm})$ than those sampled at forceout.

Table 4. FL by release or tag group for brood year (BY) 2005 hatchery-origin spring Chinook salmon from Catherine Creek (CTUIR data and ODFW, unpublished data).

\begin{tabular}{|c|c|c|c|c|c|c|}
\hline \multirow[b]{2}{*}{ Group $^{a}$} & \multicolumn{6}{|c|}{ FL (mm) } \\
\hline & Mean & SE & Range & $\mathrm{N}$ & $t$ ratio & $P>|t|$ \\
\hline $\mathrm{CaPT}$ & 109.4 & 1.2 & $88-126$ & 50 & 0.74453 & 0.4583 \\
\hline CoPT & 110.5 & 0.9 & $96-125$ & 50 & & \\
\hline PT (all) & 109.9 & 0.7 & $88-126$ & 100 & & \\
\hline PIT & 102.3 & 0.2 & 58-156 & 3,142 & & \\
\hline CaPV & 119.9 & 1.4 & $93-145$ & 60 & 0.339631 & 0.7347 \\
\hline CaPFO & 119.2 & 1.3 & $93-135$ & 60 & & \\
\hline Ca PIT & 105.6 & 0.2 & $75-156$ & 1,576 & & \\
\hline CoPV & 117.7 & 0.7 & 88-138 & 180 & 1.011363 & 0.3125 \\
\hline CoPFO & 116.7 & 0.7 & $90-153$ & 180 & & \\
\hline Co PIT & 99.0 & 0.2 & 58-117 & 1,566 & & \\
\hline
\end{tabular}

Mean weights at PIT-tagging showed similar patterns as mean FL (Table 5). Mean weights of juveniles sampled at prevolitional-release groups were greater than those sampled at pre-forceout (Table 6). Mean weights of captive and conventional broodstock progeny at pre-transfer sampling in February 2007 1.8-3.5 g greater than observed at PITtagging. 
Table 5. Weight at PIT-tagging for BY 2005 spring Chinook salmon by rearing method and release type from Catherine Creek (CTUIR data and ODFW, unpublished data).

\begin{tabular}{cccccc}
\hline & & \multicolumn{4}{c}{ Weight (g) } \\
\cline { 3 - 6 } Rearing & Release & Mean & SE & Range & $\mathrm{N}$ \\
\hline $\mathrm{H}$ & $\mathrm{CaV}$ & 15.0 & 0.7 & $11.4-21.5$ & 17 \\
$\mathrm{H}$ & CaFO & 14.8 & 2.2 & $8.6-22.0$ & 5 \\
& & & & & \\
$\mathrm{H}$ & $\mathrm{CoV}$ & 12.3 & 0.4 & $8.7-15.8$ & 24 \\
$\mathrm{H}$ & CoFO & 11.8 & 0.7 & $6.9-16.1$ & 14 \\
& & & & & \\
$\mathrm{H}$ & $\mathrm{Ca}$ & 14.1 & 0.4 & $8.6-22.0$ & 51 \\
$\mathrm{H}$ & $\mathrm{Co}$ & 11.7 & 0.3 & $6.0-16.1$ & 53 \\
& & & & & \\
$\mathrm{H}$ & All & 12.9 & 0.3 & $6.0-22.0$ & 104 \\
$\mathrm{~N}$ & All & 7.8 & 0.1 & $3.7-13.1$ & 139 \\
\hline
\end{tabular}

Table 6. Weight by release or tag group for brood year (BY) 2005 hatchery-origin spring Chinook salmon from Catherine Creek (CTUIR data and ODFW, unpublished data).

\begin{tabular}{ccccc}
\hline & \multicolumn{4}{c}{ Weight (g) } \\
\cline { 2 - 5 } Group & Mean & SE & Range & N \\
\hline CaPT & 15.9 & 0.7 & $7.1-26.0$ & 50 \\
CoPT & 15.2 & 0.6 & $7.3-27.2$ & 50 \\
PT All & 15.6 & 0.4 & $7.1-27.2$ & 100 \\
PIT & 12.9 & 0.3 & $6.0-22.0$ & 104 \\
& & & & \\
CaPV & 20.6 & 0.8 & $8.7-37.2$ & 60 \\
CaPFO & 17.5 & 0.6 & $7.2-27.4$ & 60 \\
Ca PIT & 14.1 & 0.4 & $8.6-22.0$ & 51 \\
& & & & \\
CoPV & 19.6 & 0.4 & $6.4-32.3$ & 180 \\
CoPFO & 16.6 & 0.3 & $7.4-28.6$ & 180 \\
Co PIT & 11.7 & 0.3 & $6.0-16.1$ & 53 \\
\hline
\end{tabular}

Mean K factor at PIT-tagging was significantly different between hatchery-origin and natural-origin, but none of the hatchery-origin between group comparisons were significant (Table 7). 
Table 7. K factor at PIT-tagging summary for BY 2005 spring Chinook salmon by rearing method and release type from Catherine Creek (CTUIR.data and ODFW, unpublished data).

\begin{tabular}{cccccccc}
\hline & & \multicolumn{7}{c}{ K factor } \\
\cline { 3 - 8 } Rearing & Release & Mean & SE & Range & $\mathrm{N}$ & $t$ ratio & $\mathrm{P}>|\mathrm{t}|$ \\
\hline $\mathrm{H}$ & $\mathrm{CaV}$ & 1.23 & 0.02 & $1.07-1.39$ & 17 & 1.893025 & 0.0729 \\
$\mathrm{H}$ & $\mathrm{CaFO}$ & 1.15 & 0.03 & $1.10-1.21$ & 5 & & \\
& & & & & & & \\
$\mathrm{H}$ & $\mathrm{CoV}$ & 1.17 & 0.01 & $1.04-1.28$ & 24 & -0.075461 & 0.4554 \\
$\mathrm{H}$ & $\mathrm{CoFO}$ & 1.18 & 0.02 & $1.06-1.27$ & 14 & & \\
& & & & & & & \\
$\mathrm{H}$ & $\mathrm{Ca}$ & 1.19 & 0.01 & $1.02-1.39$ & 51 & -1.65431 & 0.1011 \\
$\mathrm{H}$ & $\mathrm{Co}$ & 1.17 & 0.01 & $0.98-1.28$ & 53 & & \\
& & & & & & & \\
$\mathrm{H}$ & All & 1.18 & 0.01 & $0.98-1.39$ & 104 & -10.5353 & $<0.0001$ \\
$\mathrm{~N}$ & All & 1.05 & 0.01 & $0.86-1.52$ & 139 & & \\
\hline
\end{tabular}

There were significant differences in mean $\mathrm{K}$ factor between pre-volitional and preforceout sampling of both conventional and captive broodstock progeny, but not between the conventional and captive broodstock progeny at pretransfer sampling in February 2007 (Table 8).

Table 8. K factor by release or tag group for brood year (BY) 2005 hatchery-origin spring Chinook salmon from Catherine Creek (CTUIR data and ODFW, unpublished data).

\begin{tabular}{ccccccc}
\hline & \multicolumn{6}{c}{ K factor } \\
\cline { 2 - 7 } Group & Mean & SE & Range & $\mathrm{N}$ & $t$ ratio & $\mathrm{P}>|\mathrm{t}|$ \\
\hline CaPT & 1.18 & 0.03 & $0.87-1.83$ & 50 & -1.70862 & 0.0907 \\
CoPT & 1.11 & 0.03 & $0.76-1.88$ & 50 & & \\
PT (all) & 1.15 & 0.02 & $0.76-1.88$ & 100 & & \\
PIT & 1.18 & 0.01 & $0.98-1.39$ & 104 & & \\
& & & & & & \\
CaPV & 1.16 & 0.01 & $0.95-1.69$ & 60 & 9.358483 & $<0.0001$ \\
CaPFO & 1.01 & 0.01 & $0.84-1.23$ & 60 & & \\
Ca PIT & 1.19 & 0.01 & $1.02-1.39$ & 51 & & \\
& & & & & & \\
CoPV & 1.17 & 0.005 & $0.94-1.31$ & 180 & 20.1321 & $<0.0001$ \\
CoPFO & 1.02 & 0.01 & $0.80-124$ & 180 & & \\
Co PIT & 1.17 & 0.01 & $0.98-1.28$ & 53 & & \\
\hline
\end{tabular}




\subsubsection{Migration Timing}

There were significant differences in arrival dates at Lower Granite Dam in 2007 for all groups tested (Table 9). Median arrival date for hatchery-origin outmigrants were $3 \mathrm{~d}$ earlier than natural-origin. Median arrival dates were $5 \mathrm{~d}$ earlier for both comparisons of volitionally-released compared to forceout and $3 \mathrm{~d}$ earlier for captive broodstock progeny compared to conventional

Table 9. Arrival dates at Lower Granite Dam in 2007 of PIT-tagged BY 2005 spring Chinook salmon by rearing and release type from Catherine Creek.

\begin{tabular}{|c|c|c|c|c|c|c|c|c|}
\hline Rearing & Release & $\mathrm{N}^{*}$ & $\mathrm{~N}_{\exp } * *$ & Median & Earliest & Latest & Z value & $\mathrm{P}>|\mathrm{Z}|$ \\
\hline $\mathrm{H}$ & $\mathrm{CaV}$ & 213 & 277 & 3 May & 20 April & 18 May & -10.35 & $<0.00001$ \\
\hline $\mathrm{H}$ & $\mathrm{CaFO}$ & 732 & 913 & 8 May & 29 April & 26 May & & \\
\hline $\mathrm{H}$ & $\mathrm{CoV}$ & 410 & 523 & 7 May & 22 April & 5 June & 10.23 & $<0.00001$ \\
\hline $\mathrm{H}$ & $\mathrm{CoFO}$ & 325 & 413 & 12 May & 29 April & 7 June & & \\
\hline $\mathrm{H}$ & $\mathrm{Ca}$ & 1,024 & 1,285 & 8 May & 20 April & 8 June & -8.59 & $<0.00001$ \\
\hline $\mathrm{H}$ & Co & 1,186 & 1,480 & 11 May & 22 April & 7 June & & \\
\hline $\mathrm{H}$ & All & 2,210 & 2,750 & 10 May & 20 April & 8 June & 2.84 & 0.005 \\
\hline $\mathrm{N}$ & All & 14 & 25 & 13 May & 1 May & 25 June & & \\
\hline
\end{tabular}

The frequency distribution of arrival dates for hatchery-origin juveniles was strongly bimodal, with most arrivals between 1May and 15 May (Figure 2). The frequency distribution of arrival dates for natural origin juveniles was more evenly dispersed, although recognition of any clear pattern was tenuous due to the small sample size (Figure 3). 


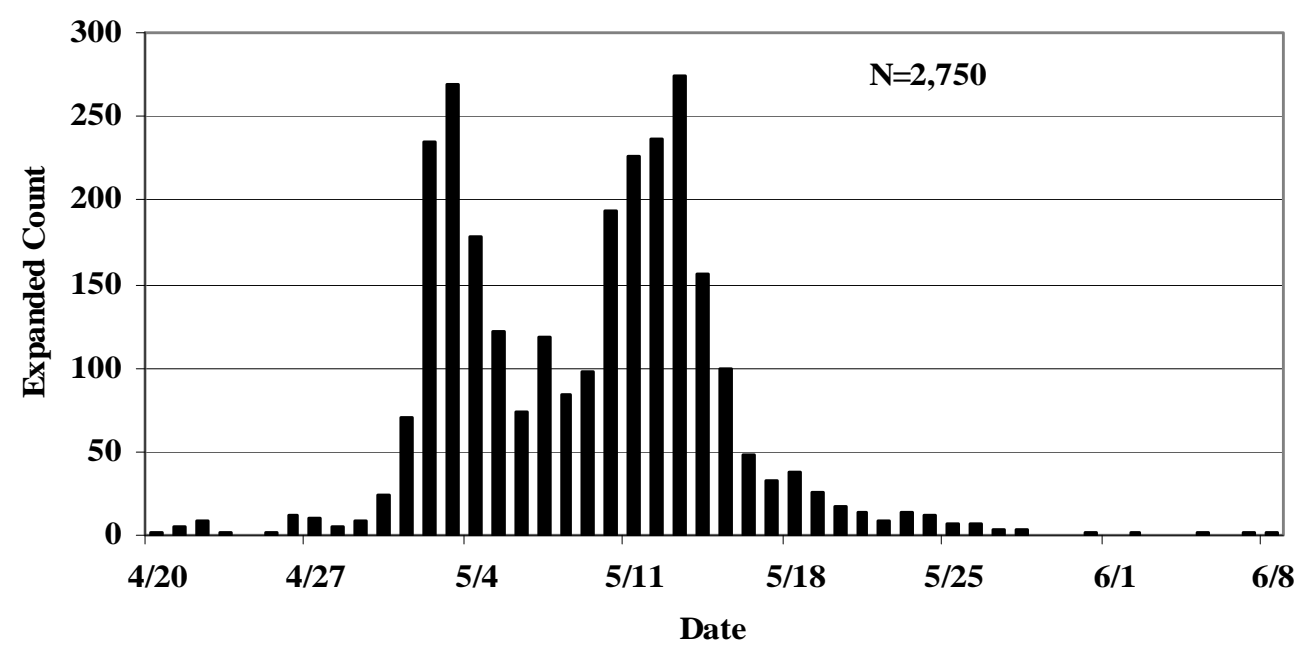

Figure 2. Frequency distribution of arrival dates (expanded for spill) at Lower Granite Dam for Catherine Creek BY 2005 hatchery-origin spring Chinook salmon.

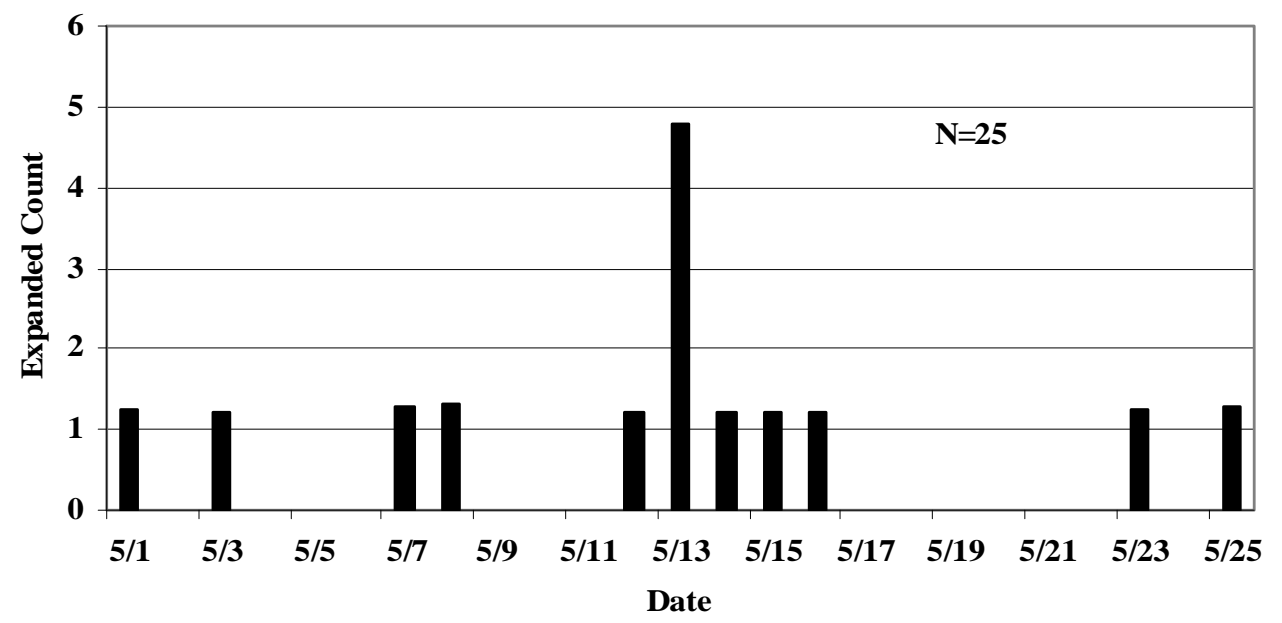

Figure 3. Frequency distribution of arrival dates (expanded for spill) at Lower Granite Dam for Catherine Creek BY 2005 natural-origin spring Chinook salmon (spring release group).

The earliest-arriving hatchery-origin outmigrants were detected at lower and more stable flows and water temperatures than the earliest-arriving natural-origin (Figure 4). Median arrival dates for both groups were close to the peak flow observed and at water temperatures of $10-12^{\circ} \mathrm{C}$. The latest-arriving hatchery-origin outmigrants were detected at higher flows and lower water temperatures than natural-origin. . 


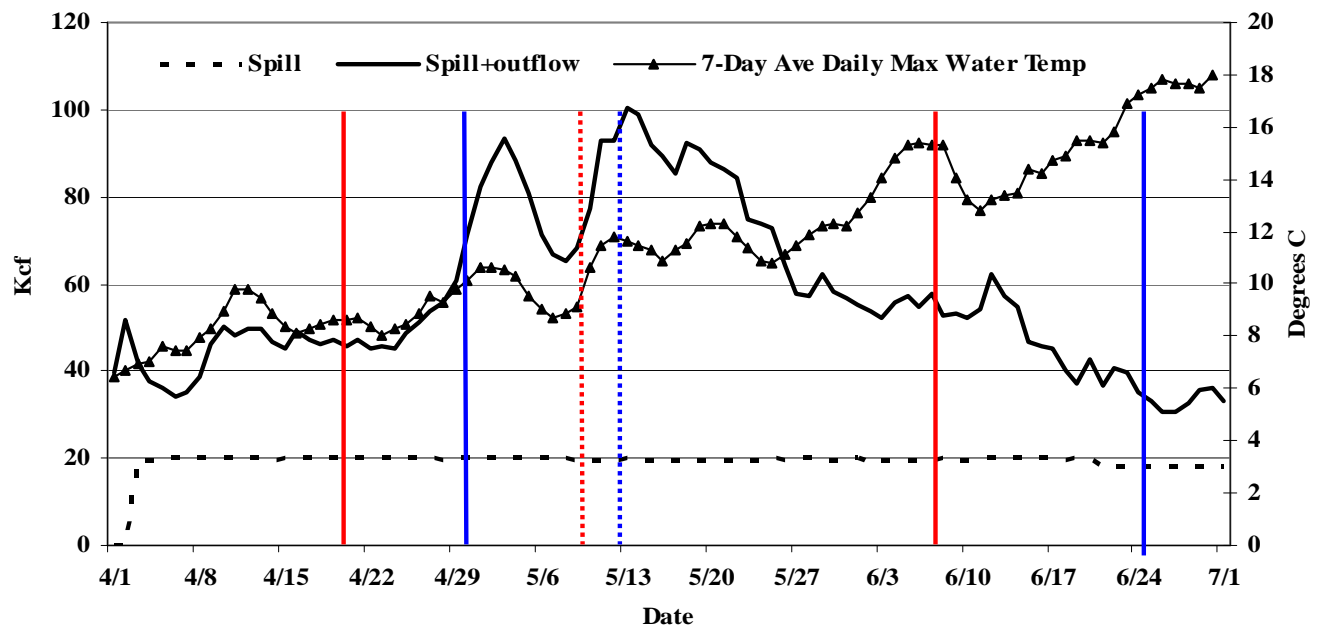

Figure 4. First and last (solid vertical lines) detection dates and median detection dates (dashed vertical lines) at Lower Granite Dam (expanded for spill) for Catherine Creek BY 2005 natural- (blue lines) and hatchery-origin (red lines) juvenile spring Chinook salmon.

As expected, travel time for volitionally-released groups was lower than for forceout (Table 10). Captive broodstock progeny had shorter (1.8 d) travel times than conventional broodstock and shorter travel time was also evident for natural-origin outmigrants.

Table 10. Harmonic means of travel time (d) to Lower Granite Dam of PIT-tagged BY 2005 spring Chinook salmon by rearing and release type from Catherine Creek.

\begin{tabular}{ccccc}
\hline Rearing & Release & Time $(\mathrm{d})$ & $\mathrm{SE}$ & $\mathrm{N}$ \\
\hline $\mathrm{H}$ & $\mathrm{CaV}$ & 38.872 & 0.371 & 212 \\
$\mathrm{H}$ & CaFO & 42.803 & 0.420 & 136 \\
& & & & \\
$\mathrm{H}$ & $\mathrm{CoV}$ & 41.699 & 0.260 & 410 \\
$\mathrm{H}$ & $\mathrm{CoFO}$ & 45.590 & 0.290 & 325 \\
& & & & \\
$\mathrm{H}$ & $\mathrm{Ca}$ & 42.271 & 0.176 & 1,023 \\
$\mathrm{H}$ & $\mathrm{Co}$ & 44.091 & 0.163 & 1,186 \\
& & & & \\
$\mathrm{H}$ & All & 43.229 & 0.121 & 2,209 \\
$\mathrm{~N}$ & All & 39.125 & 2.986 & 42 \\
\hline
\end{tabular}




\subsubsection{Survival}

Survival to Lower Granite Dam was similar for all groups, the largest difference being 0.0717 (Table 11). Based on the overlap of standard errors, there may have been a statistically significant difference between volitionally-released and forceout conventional broodstock progeny, Capture probabilities were similar between hatcheryorigin groups, the largest difference being 0.0331 . The difference between hatchery- and natural-origin outmigrants was 0.0453 .

Table 11. Survival to and capture probabilities $\left(C_{p}\right)$ at Lower Granite Dam for BY 2005 spring Chinook salmon by rearing and release type from Catherine Creek.

\begin{tabular}{ccccccc}
\hline Rearing & Release & $\mathrm{N}$ & Survival & $\mathrm{SE}$ & $\mathrm{C}_{\mathrm{p}}$ & $\mathrm{SE}$ \\
\hline $\mathrm{H}$ & $\mathrm{CaV}$ & 2,333 & 0.3529 & 0.0262 & 0.2575 & 0.0233 \\
$\mathrm{H}$ & $\mathrm{CaFO}$ & 1,562 & 0.3398 & 0.0380 & 0.2562 & 0.0331 \\
& & & & & & \\
$\mathrm{H}$ & $\mathrm{CoV}$ & 3,987 & 0.3245 & 0.0146 & 0.3169 & 0.0178 \\
$\mathrm{H}$ & $\mathrm{CoFO}$ & 2,727 & 0.3828 & 0.0237 & 0.3113 & 0.0228 \\
& & & & & & \\
$\mathrm{H}$ & $\mathrm{Ca}$ & 10,364 & 0.3348 & 0.0112 & 0.2948 & 0.0119 \\
$\mathrm{H}$ & $\mathrm{Co}$ & 10,450 & 0.3461 & 0.0097 & 0.3279 & 0.0112 \\
& & & & & & \\
$\mathrm{H}$ & All & 20,814 & 0.3249 & 0.0455 & 0.3266 & 0.0459 \\
$\mathrm{~N}$ & $\mathrm{All}$ & 363 & 0.3111 & 0.0356 & 0.3719 & 0.0551 \\
\hline
\end{tabular}

\subsubsection{Catherine Creek Adult Spring Chinook Salmon}

\subsubsection{Harvest}

Brood year 2002 releases from the Catherine Creek acclimation facility were marked with CWT and adipose clips. Conventional broodstock progeny also received VIE tags. CWT recoveries of BY 2002 Catherine Creek stock were almost entirely from Catherine Creek, either at the trap or on spawning ground surveys (Table 12). There were no recoveries in Columbia River sport or commercial fisheries at age 3 or 5 . There was one recovery age 4 by the Washington Department of Fish and Wildlife (expanded to 2.44) in the Snake River sport fishery. One age 4 recovery (expanded to 4.45) occurred in the ocean troll fishery. 
Table 12. Estimated tag recoveries of BY 2002 spring Chinook salmon in the Columbia River in 2005-2007 that were released from the Catherine Creek acclimation facility.

\begin{tabular}{cccc}
\hline Tag code & Run Year & Recovery Location & Estimated Number \\
\hline 93835 & 2005 & CC SGS & 1 \\
93835 & 2005 & CC Trap & 4.08 \\
93836 & 2005 & CC Trap & 1.02 \\
93840 & 2005 & CC Trap & 19.38 \\
& RY 2005 Total & & 25.48 \\
93835 & 2006 & CC SGS & 8 \\
93836 & 2006 & CC SGS & 8 \\
93836 & 2006 & LKG SGS & 1 \\
93840 & 2006 & CC SGS & 32 \\
93840 & 2006 & CC Trap & 48 \\
93840 & 2006 & LKG SGS & 2 \\
93840 & 2006 & NWTR 120-00 & 4.45 \\
93840 & 2006 & Snake River Mainstem & 2.44 \\
& RY 2006 Total & & 105.89 \\
93836 & 2007 & CC SGS & 1 \\
& RY 2007 Total & & 1 \\
\hline
\end{tabular}

\subsubsection{Trap Catch}

The Catherine Creek trap was operated from 1 March-31 July 2007 and a total of 242 adults were caught (115 females, 127 males). Disposition of natural-origin adults included passing above the trap to spawn, trap mortalities, or transporting to Lookingglass Hatchery for use as conventional broodstock. Disposition of hatcheryorigin adults included trap mortalities, removal (euthanizing) of jacks to maintain desired hatchery:natural ratios, transporting to Lookingglass Hatchery for use as broodstock, and passing above the trap to spawn. Only conventional broodstock progeny were used for spawning at Lookingglass Hatchery.

Natural-origin adults were collected from 14 May-25 June and hatchery-origin from 21 May-25 June. None had radio tags; one hatchery-origin was confirmed as a stray by a PIT tag (tagged at BONAFF). Total catches of natural- and hatchery-origin adults were 77 (36 females, 41 males) and 165 (79 females, 86 males), respectively. Cumulative frequency distributions for arrival timing showed a slightly greater percentage of naturalorigin adults arrived early in the run (Figure 5) but the Kolmogorov-Smirnov test for a difference in arrival date distributions was not significant (2-tailed K-S statistic $=0.13$, $\mathrm{P}=0.3093)$. 


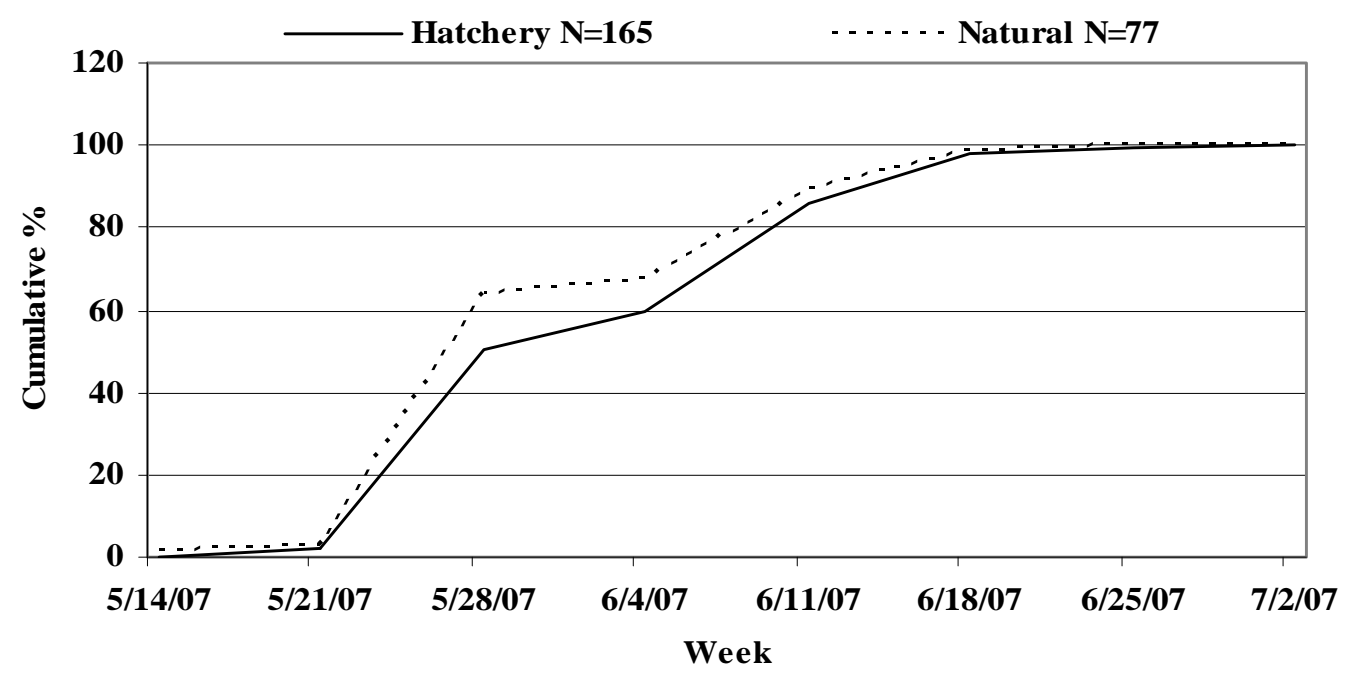

Figure 5. Cumulative percentages of natural- and hatchery-origin spring Chinook salmon caught by week of the year and age group at the Catherine Creek trap, 2007.

Minimum water temperatures ranged from $4.6-12.8^{\circ} \mathrm{C}$ and 7 - day average maximum water temperatures from $6.1-19.7^{\circ} \mathrm{C}$ during the period when spring Chinook were captured (Figure 6). There was nothing conspicuous regarding water temperatures for the week of peak capture (28 May) when approximately $53 \%$ of the total catch occurred.

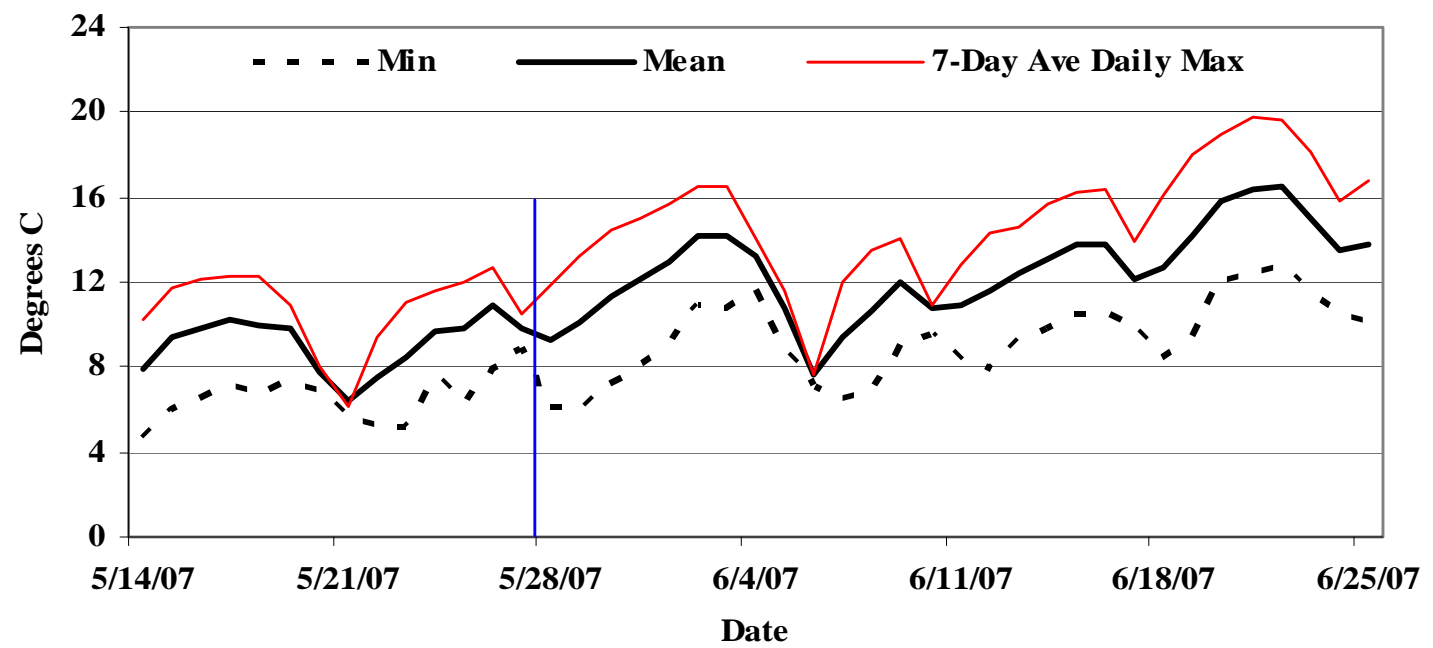

Figure 6. Water temperatures at the Catherine Creek adult trap encompassing the dates of first and last captures of spring Chinook salmon, 2007 (blue vertical line=week of peak catch) . 
Conventional broodstock progeny (36 females, 27 males) were collected from 21 May-22 June. Captive broodstock progeny (43 females, 59 males) were collected from 21 May25. One was collected at an irrigation dam below the city of Union and released above the trap.

The length frequency for hatchery-origin returns was dominated by the 64-78 cm groups (Figure 7). The length frequency for natural-origin adults (Figure 8) showed higher percentages of large returns $(>80 \mathrm{~cm})$ and a lower percentage of small returns $(<60 \mathrm{~cm})$ compared to hatchery-origin. The Kolmogorov-Smirnov test for a difference in FL (cm) distributions between hatchery- and natural-origin adults was significant (2-tailed K-S statistic $=0.29, \mathrm{P}=0.0003)$.

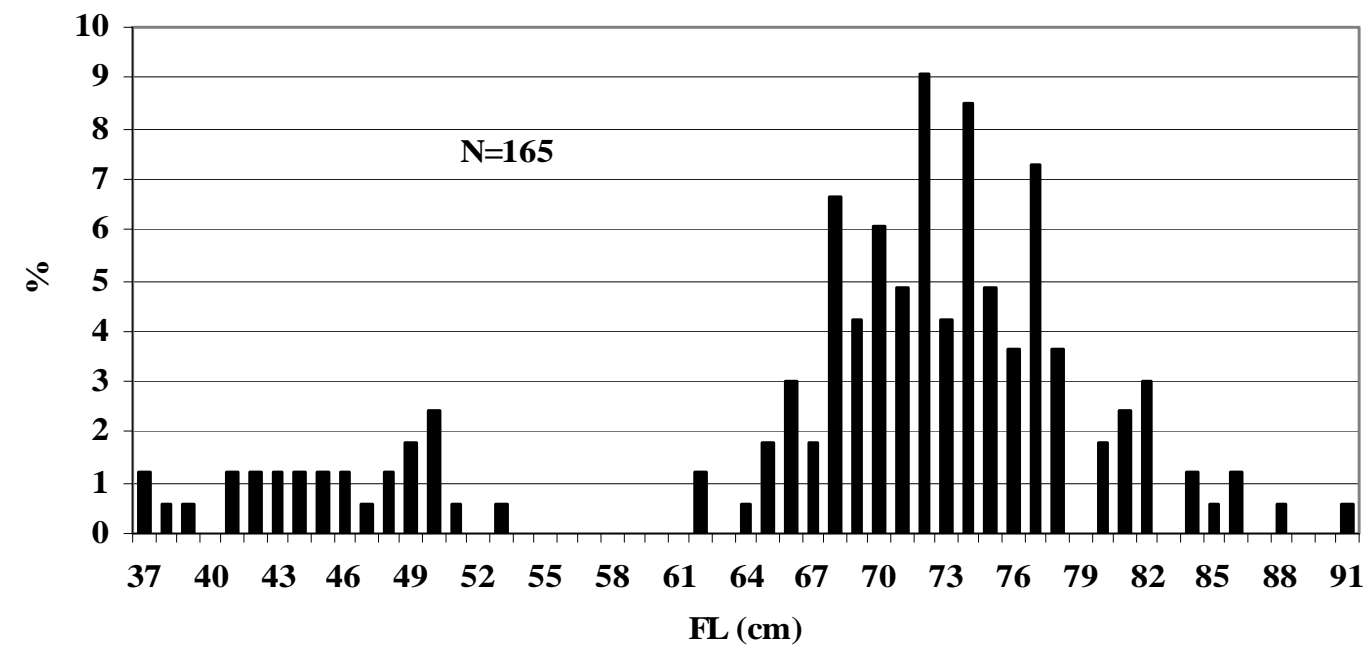

Figure 7. FL distribution of hatchery-origin spring Chinook salmon caught at the Catherine Creek trap, 2007. 


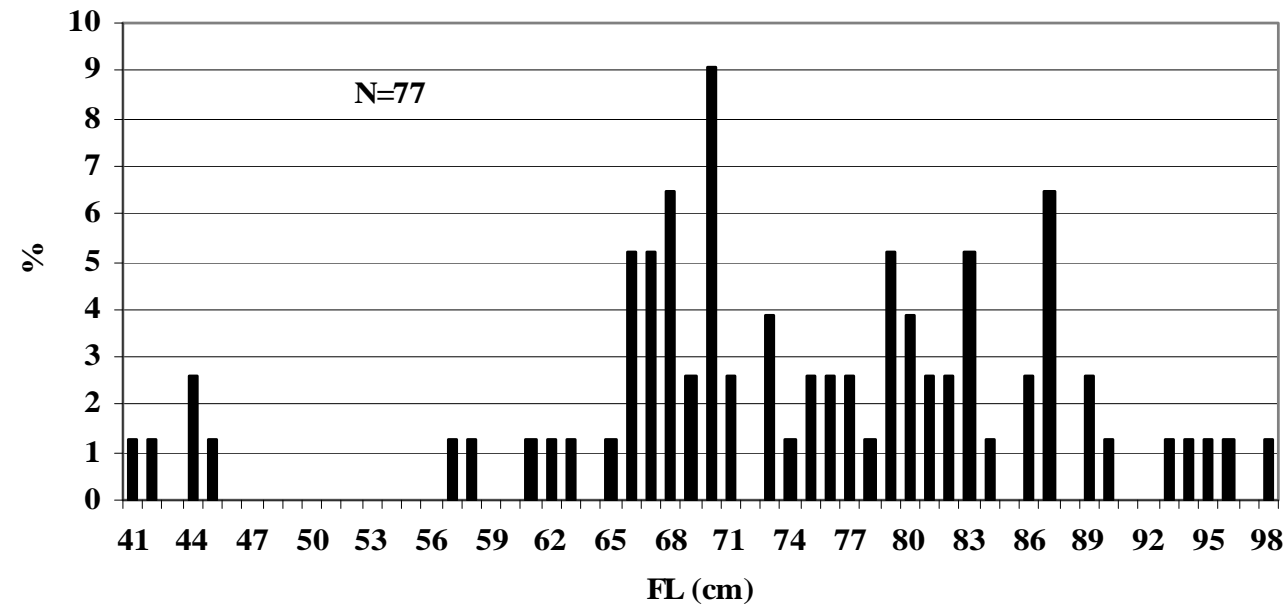

Figure 8. FL distribution of natural-origin spring Chinook salmon caught at the Catherine Creek trap, 2007.

Conventional broodstock progeny were primarily in the $65-78 \mathrm{~cm}$ size groups (Figure 9), with a more restricted size distribution than captive broodstock (Figure 10). Captive broodstock progeny euthanized at the trap ranged from 37-50 cm (Figure 11).

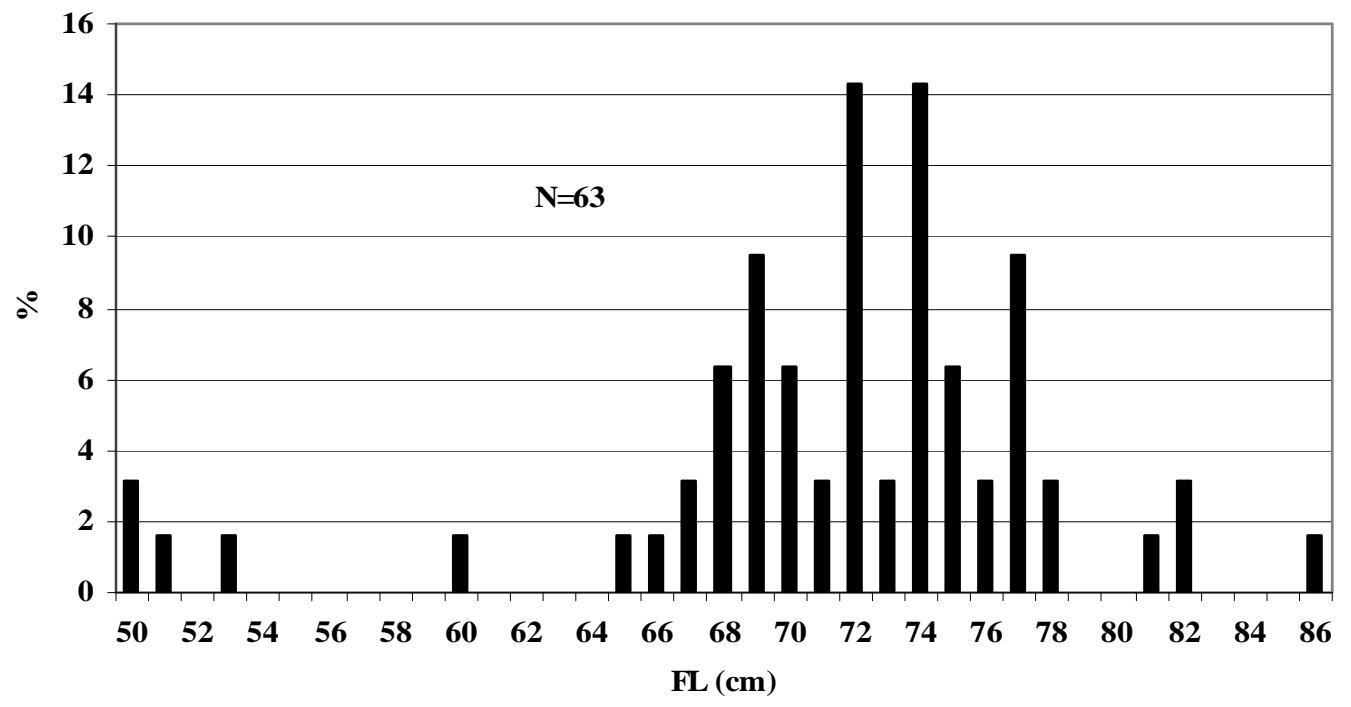

Figure 9. FL distribution of hatchery-origin (conventional broodstock progeny) spring Chinook salmon caught at the Catherine Creek trap, 2007. 


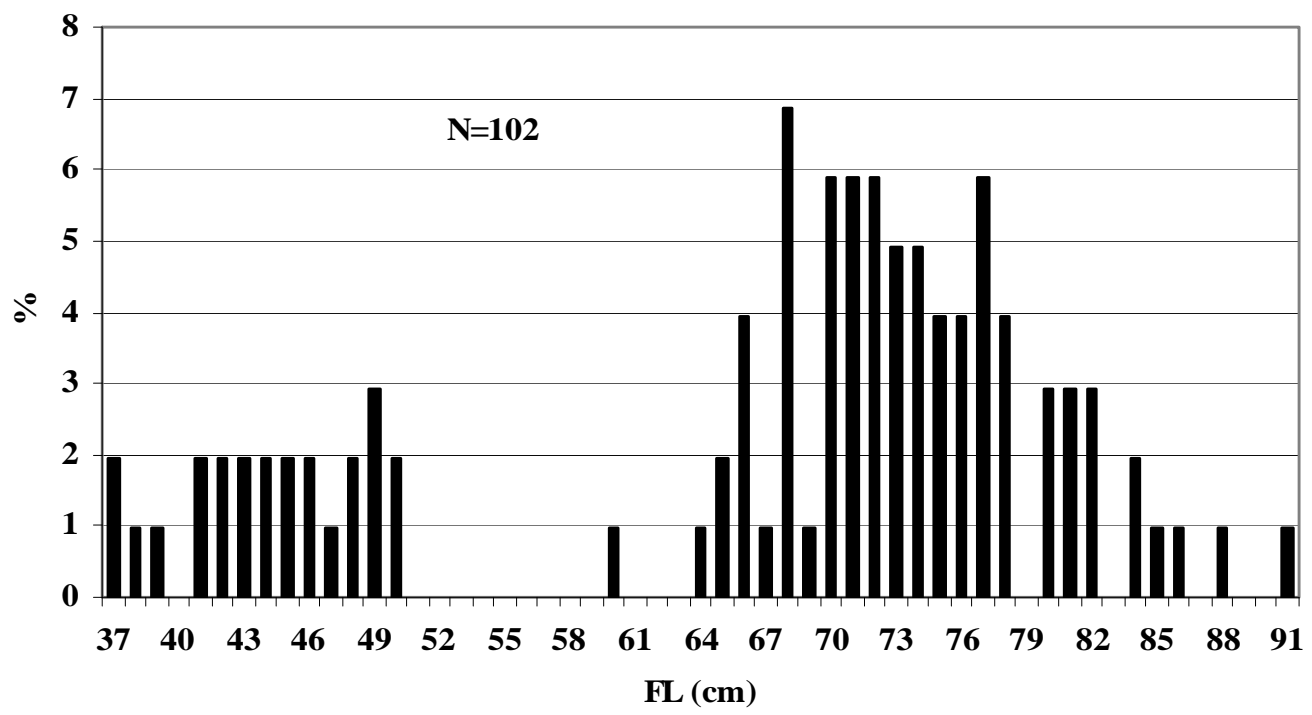

Figure 10. FL distribution of hatchery-origin (captive broodstock progeny) spring Chinook salmon caught at the Catherine Creek trap, 2007.

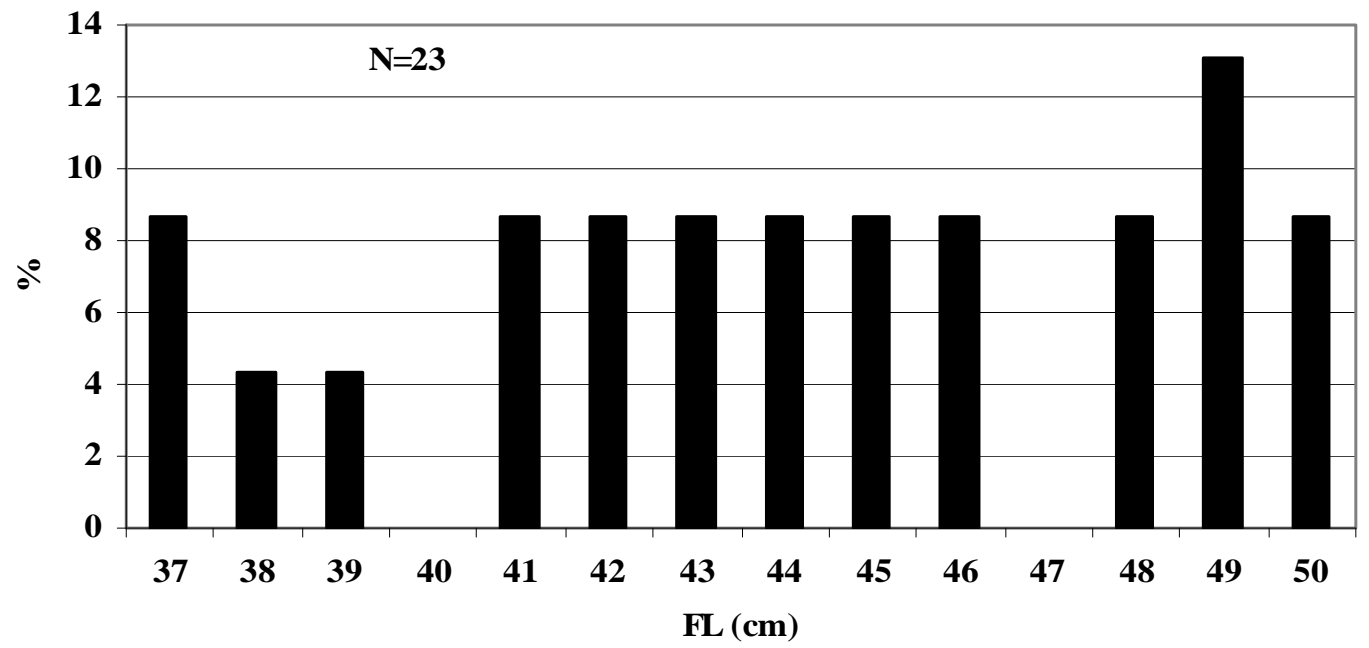

Figure 11. FL distribution of hatchery-origin (captive broodstock progeny) spring Chinook salmon caught at the Catherine Creek trap and euthanized, 2007. 


\subsubsection{Conventional Spawning}

Broodstock spawned at Lookingglass Hatchery in 2007 included 28 natural- (14 females, 14 males) and 47 hatchery-origin (31 females, 16 males). Two hatchery-origin females and one natural-origin male died while being held at the hatchery prior to spawning. Natural-origin females were spawned on 22 August (1), 29 August (9), and 6 September (4) and hatchery-origin on 22 August (1), 29 August (15), 6 September (8), and 11 September (7). There was no significant difference in the spawn timing between hatchery- and natural-origin females (Wilcoxon test, $\mathrm{Z}=-1.60518, \mathrm{P}>|\mathrm{Z}|=0.1085$ ). More large females $(>80 \mathrm{~cm}$ ) were spawned from the natural-origin group (Figure 12).

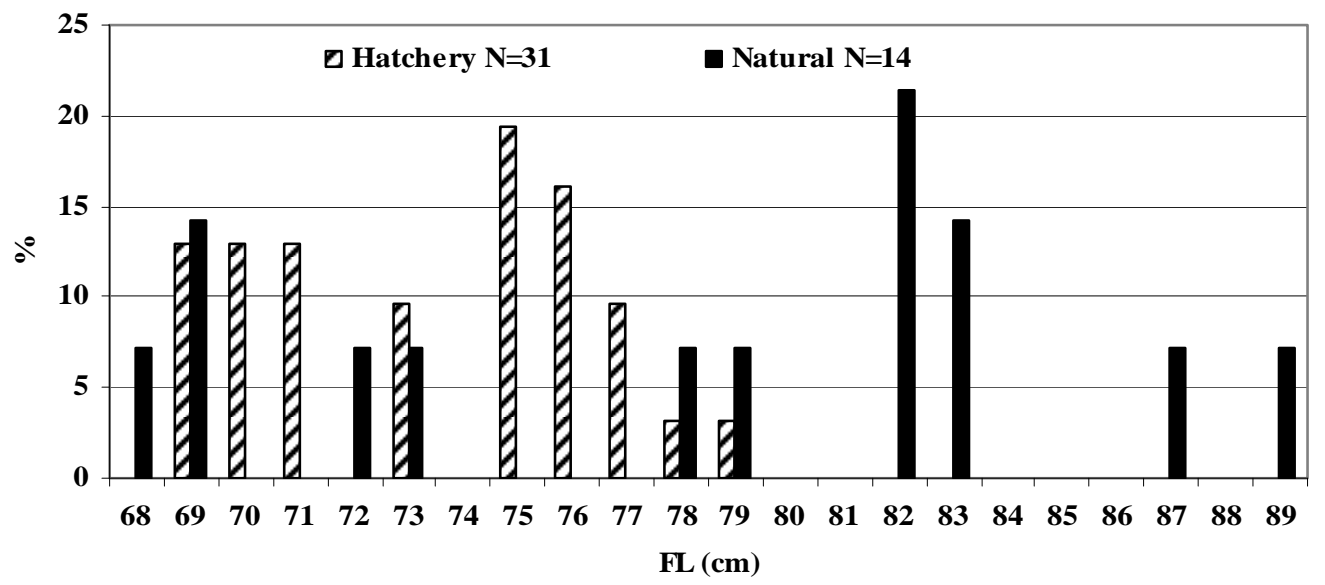

Figure 12. FL distribution for female Catherine Creek conventional broodstock by origin, 2007.

Mean FL of natural-origin spawners was greater than hatchery-origin (Table 13). Mean FL of hatchery- and natural-origin males spawned were similar $(\mathrm{t}=1.541691, \mathrm{P}>|\mathrm{t}|=$ $0.1352)$. Mean FL of hatchery- and natural-origin females spawned were also similar ( $\mathrm{t}=$ $-0.74537, \mathrm{P}>|\mathrm{t}|=0.4620$ ). Mean weight of natural-origin females spawned was $0.9 \mathrm{~kg}$ greater than for hatchery-origin (Table 14). An apparently erroneous weight for a 691 mm FL hatchery-origin female was omitted from results in Tables 13 and 14. 
Table 13. FL summary for Catherine Creek conventional broodstock spawned in 2007.

\begin{tabular}{cccccc}
\hline Origin & Sex & Mean FL(mm) & SE & Range & N \\
\hline Hatchery & M & 709.6 & 29.8 & $495-844$ & 16 \\
Natural & M & 767.4 & 35.7 & $415-950$ & 14 \\
Hatchery & F & 738.6 & 5.6 & $691-793$ & 31 \\
Natural & F & 788.3 & 18.7 & $682-895$ & 14 \\
\hline
\end{tabular}

Table 14. Weight summary for female Catherine Creek conventional broodstock spawned in 2007.

\begin{tabular}{ccccc}
\hline Origin & Mean Wgt $(\mathrm{kg})$ & $\mathrm{SE}$ & Range & $\mathrm{N}$ \\
\hline Hatchery & 4.0 & 0.1 & $3.05-5.3$ & 19 \\
Natural & 4.9 & 0.3 & $2.54-6.50$ & 12 \\
\hline
\end{tabular}

The correlation between FL and weight was better for natural- than hatchery-origin spawners (Table 15). Predicted weights for $750 \mathrm{~mm}$ FL hatchery- and natural-origin females were both $4.1 \mathrm{~kg}$.

Table 15. $\log _{10}$ length-weight regression summary for female Catherine Creek conventional broodstock spawned in 2007.

\begin{tabular}{ccccc}
\hline Origin & Slope & Intercept & Adj. $\mathrm{R}^{2}$ & $\mathrm{~N}$ \\
\hline Hatchery & 2.651534 & -7.011256 & 0.630235 & 19 \\
Natural & 2.834939 & -7.534595 & 0.809183 & 12 \\
\hline
\end{tabular}

Mean fecundity was slightly higher for natural-origin than hatchery-origin (Table 16) and the difference in mean fecundity between the two rear types was significant ( $\mathrm{t}$ value = $2.465445, P>|t|=0.0179$ ). Fecundity for a $694 \mathrm{~mm}$ FL, $2.54 \mathrm{~kg}$ natural-origin female was clearly an outlier, so data from this fish were not included in testing mean fecundity between hatchery- and natural-origin or in the analysis of covariance.

Mean egg weight was $0.23 \mathrm{~g}$ (range $0.16-0.27$ ) for 31 hatchery- and $0.24 \mathrm{~g}$ (range 0.19 0.30 ) for 14 natural-origin females. There was no significant difference in mean egg weight between the two rear types ( $\mathrm{t}$ value $=1.417306, \mathrm{P}>|\mathrm{t}|=0.1638)$. 
Table 16. Fecundity (eggs/female) and egg weight summary for Catherine Creek conventional broodstock spawned in 2007.

\begin{tabular}{ccccccc}
\hline Origin & Mean & SE & Range & N & Mean egg weight (g) & N \\
\hline Hatchery & 3,746 & 76 & $3,173-5,045$ & 31 & 0.23 & 31 \\
Natural & 3,924 & 263 & $1,158-5,226$ & 14 & 0.24 & 14 \\
\hline
\end{tabular}

\subsubsection{Spawning Ground Surveys}

Two prespawning surveys were conducted in 2007 on the 7735 Bridge to 203 Highway Bridge (12 July) and 203 Highway Bridge to Badger Flat (17 August) sections, yielding observations of 5 live fish, but no redds or carcasses. Standard spawning ground surveys were conducted on 28 August, 5 September, and 11 September; 59 redds were counted, 57 above the trap and 2 below (ODFW, unpublished data). The population estimate of spawners above the trap was 159 (ODFW, unpublished data) and 5 below. Carcass recoveries totaled 22, 21 above the trap (Table 17). One female (unclipped, $830 \mathrm{~mm}$ FL $100 \%$ spawned-out) was recovered on 5 September below the trap. Total carcass recoveries above the trap were $14 \%$ of the number released (hatchery-origin - $16 \%$, natural-origin-10\%). Roughly twice as many hatchery-origin fish were released above the trap as natural. Roughly equal numbers of males and females were released above the trap, but females dominated the hatchery-origin carcass recoveries. 
Table 17. Natural- and hatchery-origin spring Chinook salmon released above the Catherine Creek weir and carcass recoveries above the weir in 2007 by FL group, sex, and origin.

\begin{tabular}{|c|c|c|c|c|c|c|c|c|}
\hline \multicolumn{4}{|c|}{ Passed } & \multirow{3}{*}{$\underline{\text { FL (cm) Group }}$} & \multicolumn{4}{|c|}{ Recovered } \\
\hline \multicolumn{2}{|c|}{ Natural } & \multicolumn{2}{|c|}{ Hatchery } & & \multicolumn{2}{|c|}{ Natural } & \multicolumn{2}{|c|}{ Hatchery } \\
\hline $\mathrm{M}$ & $\mathrm{F}$ & $\mathrm{M}$ & $\mathrm{F}$ & & $\mathrm{M}$ & $\mathrm{F}$ & $\mathrm{M}$ & $\mathrm{F}$ \\
\hline 3 & & & & $40-44$ & 1 & & & \\
\hline 1 & & 1 & & $45-49$ & & & & \\
\hline & & & & $50-54$ & & & & \\
\hline 2 & & & & $55-59$ & & & & \\
\hline 1 & 2 & & 3 & $60-64$ & & & & 1 \\
\hline 6 & 6 & 8 & 14 & $65-69$ & & 1 & & 4 \\
\hline 2 & 5 & 19 & 17 & $70-74$ & & 1 & 1 & 5 \\
\hline 2 & 3 & 10 & 12 & $75-79$ & 2 & & 1 & 2 \\
\hline 3 & 3 & 7 & 5 & $80-84$ & & & 2 & \\
\hline 3 & 2 & 1 & 3 & $85-89$ & & & & \\
\hline 1 & 2 & 1 & & $90-94$ & & & & \\
\hline 1 & & & & & & & & \\
\hline 25 & 23 & 47 & 54 & Totals & 3 & 2 & 4 & 12 \\
\hline
\end{tabular}

Out of 12 hatchery-origin females examined for percent spawned, 11 were $100 \%$ spawned out, and 1 was $75 \%$ spawned out. Both natural-origin females examined were $100 \%$ spawned out.

Hatchery-origin female carcasses were found in three sections (Figure 13), and recoveries were on 28 August (1), 5 September (5), and 11 September (6). The two natural-origin females were recovered on 5 September. 


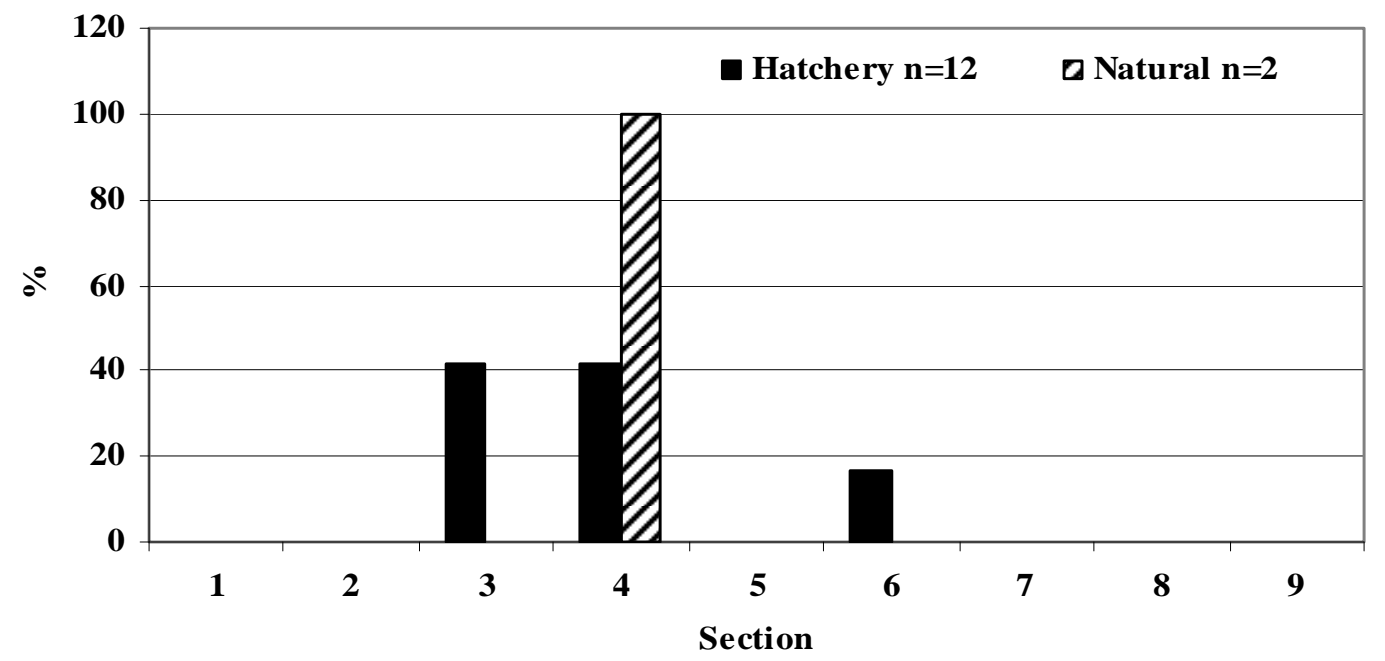

Sections

1 - North Fork Campground to Forks, 2 - South Fork, 3 - Forks to 7735 Bridge, 4 - 7735 Bridge to 203 Bridge, 5 - 203 Bridge to Badger Flat, 6-Badger Flat to Culvert at MP 5, 7-Culvert to top of Southern Cross, 8 - Bottom of Southern Cross to Weir, 9 - Weir to $2^{\text {nd }}$ bridge in Union

Figure 13. Female spring Chinook salmon carcass recoveries from Catherine Creek by section and origin, 2007.

\subsubsection{Run Reconstruction}

The age-length key developed for natural-origin returns collected at the trap in 2007 was dominated by ages 4 and 5 (Table 18). Ages 4 and 5 were approximately equal in the scale samples aged. Age 4 made up $89 \%$ of the sample of CWT from hatchery-origin returns collected at the trap or on spawning ground surveys (Table 19).

Hatchery-origin returns dominated escapement (Table 20). Total returns were dominated by hatchery-origin (Table 19). Returns of ages 4 and 5 showed patterns similar to the samples aged by scales and CWT, i.e. hatchery-origin dominated by age 4 with ages 4 and 5 natural-origin about equal. 
Table 18. Age-length table for natural-origin spring Chinook salmon collected at the Catherine Creek trap, 2007.

\begin{tabular}{|c|c|c|c|c|}
\hline FL (cm) & Age 3 & Age 4 & Age 5 & Totals \\
\hline 42 & 1 & & & 1 \\
\hline 44 & 2 & & & 2 \\
\hline 45 & 1 & & & 1 \\
\hline 57 & 1 & & & 1 \\
\hline 58 & & 1 & & 1 \\
\hline 61 & & 1 & & 1 \\
\hline 62 & & 1 & & 1 \\
\hline 63 & & 1 & & 1 \\
\hline 66 & & 3 & & 3 \\
\hline 67 & & 3 & & 3 \\
\hline 68 & & 4 & & 4 \\
\hline 69 & & 2 & & 2 \\
\hline 70 & & 3 & & 3 \\
\hline 71 & & 1 & & 1 \\
\hline 73 & & 3 & & 3 \\
\hline 76 & & 1 & 1 & 2 \\
\hline 77 & & & 1 & 1 \\
\hline 79 & & & 2 & 2 \\
\hline 80 & & & 1 & 1 \\
\hline 81 & & & 1 & 1 \\
\hline 82 & & 1 & & 1 \\
\hline 83 & & 1 & 1 & 2 \\
\hline 84 & & & 1 & 1 \\
\hline 86 & & & 2 & 2 \\
\hline 87 & & & 2 & 2 \\
\hline 89 & & & 1 & 1 \\
\hline 90 & & & 1 & 1 \\
\hline 93 & & & 1 & 1 \\
\hline 94 & & & 1 & 1 \\
\hline 96 & & & 1 & 1 \\
\hline Totals & 5 & 26 & 22 & 48 \\
\hline
\end{tabular}


Table 19. Age-length table for hatchery-origin spring Chinook salmon collected at the Catherine Creek trap or on spawning ground surveys, 2007.

\begin{tabular}{ccccc}
\hline FL $(\mathrm{cm})$ & Age 3 & Age 4 & Age 5 & Totals \\
\hline 41 & 1 & & & 1 \\
49 & 1 & & & 1 \\
50 & 1 & & & 1 \\
54 & 2 & & & 2 \\
55 & 1 & & & 1 \\
65 & & 3 & & 3 \\
67 & & 2 & & 2 \\
68 & & 3 & & 3 \\
69 & & 5 & & 5 \\
70 & & 7 & & 7 \\
71 & & 5 & & 5 \\
72 & & 2 & & 2 \\
73 & & 4 & & 4 \\
74 & & 2 & & 2 \\
75 & & 5 & & 5 \\
76 & & 4 & & 4 \\
77 & & 3 & & 3 \\
78 & & 3 & & 3 \\
79 & & 3 & & 3 \\
80 & & 2 & & 2 \\
81 & & 1 & & 1 \\
82 & & 2 & & 2 \\
84 & & 1 & 1 & 2 \\
88 & & 1 & & 1 \\
Totals & 6 & 58 & 1 & 65 \\
\hline & & & &
\end{tabular}


Table 20. Catch at the Catherine Creek trap (CCT), disposition, and estimated total returns for adult spring Chinook salmon to Catherine Creek*, 2007.

\begin{tabular}{|c|c|c|c|c|c|c|c|}
\hline Stock/Rear ${ }^{\mathrm{a}}$ & Age $^{b}$ & CCT & Euthanized $^{\mathrm{C}}$ & Spawn Ab-CCT ${ }^{\mathrm{d}}$ & Spawn LH ${ }^{\mathrm{e}}$ & Spawn Bel-CCT ${ }^{f}$ & Total Returns \\
\hline $\mathrm{CC}$ & 3 & 30 & 25 & 3 & 2 & 1 & 31 \\
\hline Natural & 3 & 6 & & 5 & 1 & aa & 6 \\
\hline Totals & & 36 & 25 & 8 & 3 & 1 & 37 \\
\hline $\mathrm{CC}$ & 4 & 128 & & 103 & 37 & 3 & 143 \\
\hline Natural & 4 & 37 & & 28 & 12 & 1 & 41 \\
\hline Totals & & 165 & & 131 & 49 & 4 & 184 \\
\hline $\mathrm{CC}$ & 5 & 7 & & 8 & & aa & 8 \\
\hline Natural & 5 & 34 & & 20 & 16 & 1 & 37 \\
\hline Totals & & 41 & & 28 & 16 & 1 & 45 \\
\hline
\end{tabular}

* Rounded to nearest whole number; $a a=<1$

${ }^{a} \mathrm{C} C=$ Catherine Creek hatchery-origin, Natural $=$ Unmarked

${ }^{b}$ Age $3 \mathrm{CC}<62 \mathrm{~cm} \mathrm{FL}$, age $4 \mathrm{CC} \geq 62 \leq 82 \mathrm{~cm} \mathrm{FL}$, age $5 \mathrm{CC} \geq 83 \mathrm{~cm} \mathrm{FL}$, age 3 Natural $<57 \mathrm{~cm} \mathrm{FL,} \mathrm{age} 4$ Natural $\geq 58 \leq 76 \mathrm{~cm}$ FL, age $5 \mathrm{Natural}>76 \mathrm{~cm} F L$

${ }^{c}$ Euthanized to maintain desired hatchery/natural ratio of spawners above the trap

${ }^{d}$ Spawn Ab-CCT=estimated spawners above the CCT trap

${ }^{e}$ Spawn LH=conventional broodstock spawned at Lookingglass Hatchery or mortality while being held for spawning

${ }^{f}$ Spawn Bel-CCT=estimated spawners below CCT, from reddsx2.5 fish/redd and catch by age,stock/rear group at CCT 
Hatchery-origin returns were all Catherine Creek stock that had been released into Catherine Creek (Table 21). Only 1 CWT code each was present for ages 3 and 5. Ages 3 and 5 tag groups were captive broodstock progeny, but age 4 returns and instream spawners were made up of both captive broodstock progeny (Ad-clip and CWT) and conventional broodstock progeny (Ad-clip+CWT+VIE). The totals of returns to stream and instream spawners in Table 21 were adjusted to account for both conventional and captive broodstock returns released above the trap, compared to only conventional broodstock progeny returns removed and taken to Lookingglass Hatchery to be spawned. CWT groups for instream spawners were allocated using only those from carcasses recovered on spawning ground surveys, while CWT groups for returns taken to the hatchery were allocated using only those with return location name listed as "Catherine Creek Trap". Total returns to the stream and instream spawners in Table 21 do not sum to 143 and 106, respectively (Table 20) due to rounding.

Table 21. Estimated hatchery-origin adult spring Chinook salmon returns to Catherine Creek and instream spawners* for 2007, by age, mark(s), and CWT group.

\begin{tabular}{ccccc}
\hline Age & Mark & CWT & Returns to Stream & Instream spawners \\
\hline 3 & Ad-clip & 94218 & 31 & 4 \\
& & & & 29 \\
4 & Ad-clip/VIE & 70753 & 44 & 39 \\
4 & Ad-clip/VIE & 70754 & 61 & 39 \\
4 & Ad-clip & 94040 & 39 & 8 \\
& & & & \\
5 & Ad-clip & 93836 & 8 & \\
\hline
\end{tabular}

* Rounded to nearest whole number; $a a=<1$

Smolt-to-adult ratio of returns to the stream for ad-clipped juveniles released in the spring of 2004 was $246 / 161,868$ or $0.15 \%$. The smolt-to-adult ratio for naturally-produced smolt equivalents (outmigrants adjusted for survival to Lower Granite Dam) was 158/3,302 or 5.40\%. Progeny-per-parent for brood year 2002 (all age 4/5 instream spawners in 2002, age 4 natural returns in 2006 and age 5 natural returns in 2007) was 0.36 .

\subsubsection{Run Size Predictors}

We used Catherine Creek returns to the stream data for the years 2001-2007 to determine if returns at age 3 could be used to predict age 4 returns the following year. The least squares regression for hatchery-origin returns using all available data was not significant (Table 22). The catches of brood year 2004 appeared to be an outlier (Figure 14). If this data point was omitted, the regression became significant. Age 3 natural-origin returns 
were poorly correlated with age 4 returns (Figure 15). Age 3 returns for 5 of 6 years ranged only from 4-10, with age 4 returns a year later ranging from 41-119. Removing the brood year 2001 data point that had the highest age 3 and age 4 returns did not improve the relationship.

Table 22. Regression statistics using age 3 returns to predict age 4 or both age 4 and age 5 returns combined for the same brood year.

\begin{tabular}{llcccc}
\hline Origin & $\mathrm{N}$ & Regression formula & Adjusted $^{2}$ & F-ratio & $\mathrm{P}>|\mathrm{F}|$ \\
\hline Hatchery & 6 & Age $4=87.548+4.196 *$ Age 3 & 0.133 & 1.768 & 0.2544 \\
Hatchery & 5 & Age 4 $=51.730+7.066 *$ Age 3 & 0.943 & 67.361 & 0.0038 \\
\hline
\end{tabular}

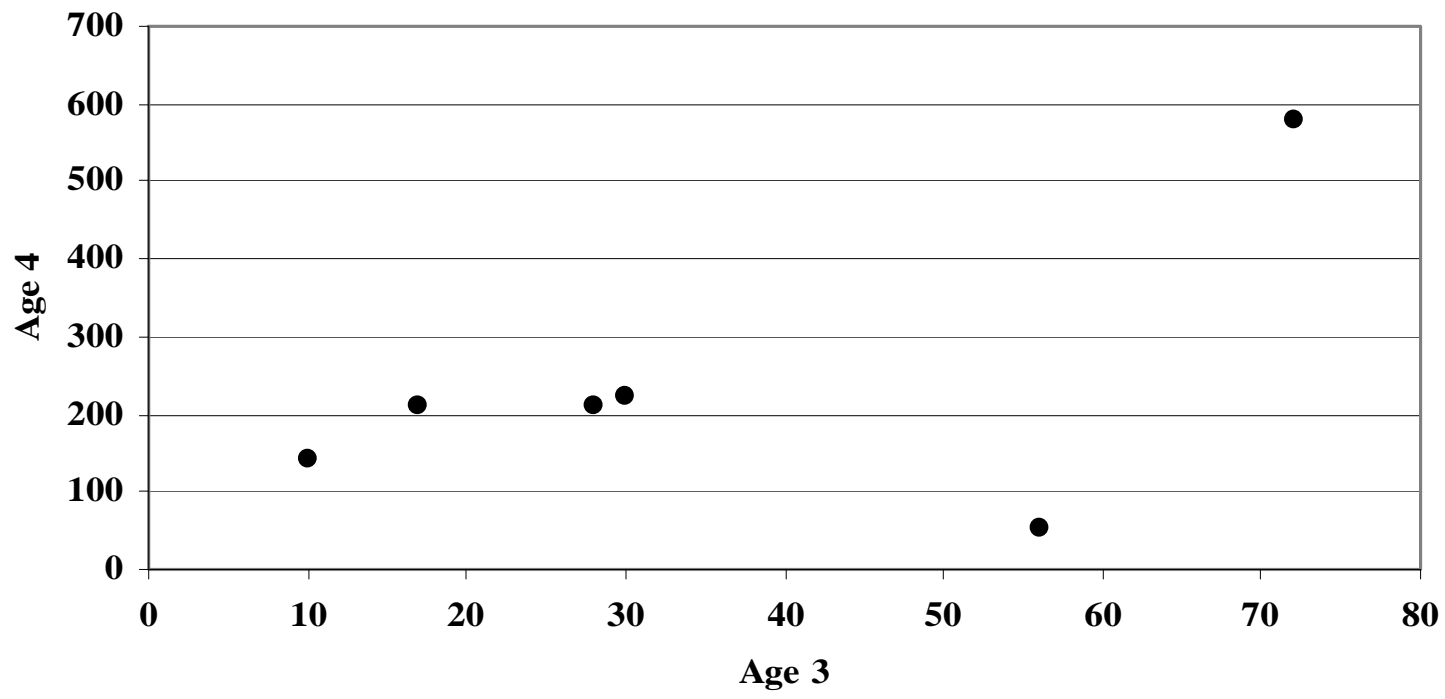

Figure 14. Scatter plot of Catherine Creek hatchery-origin age 3 vs. age 4 spring Chinook salmon returns for run years 2001-2006. 


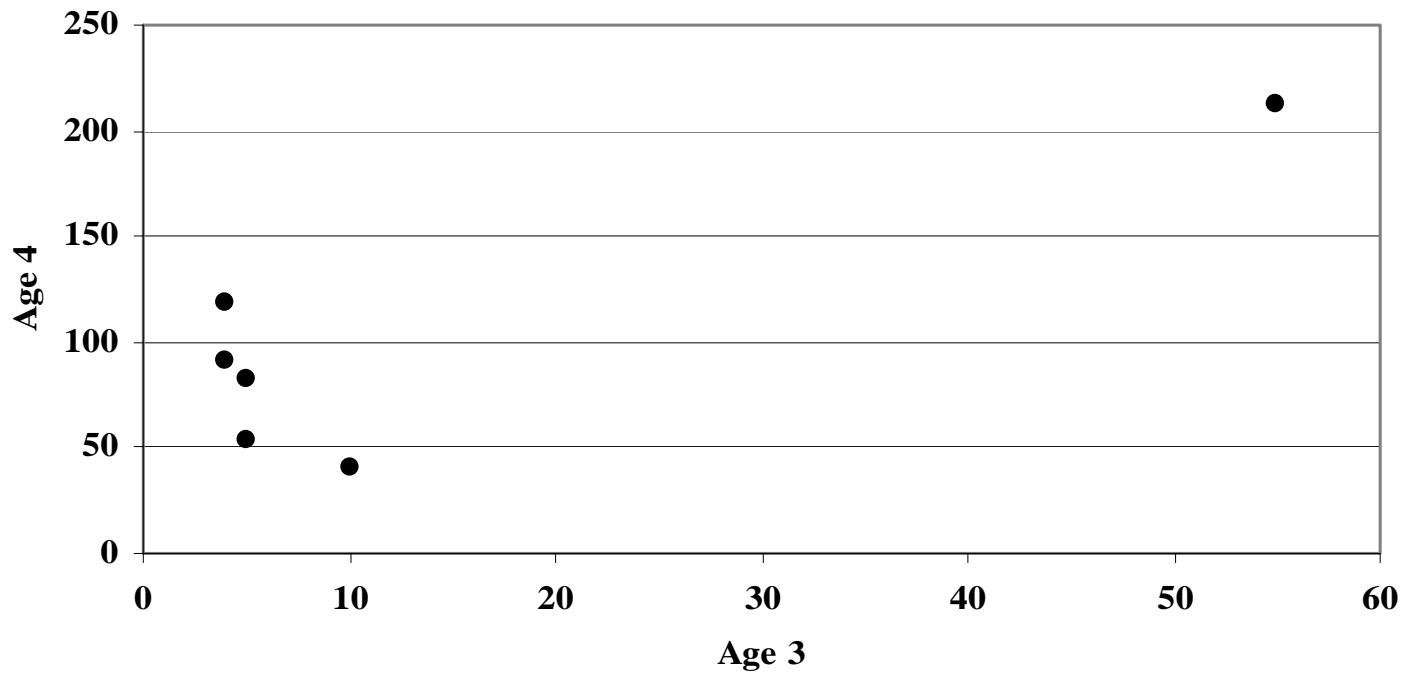

Figure 15. Scatter plot of Catherine Creek natural-origin age 3 vs. age 4 spring Chinook salmon returns for run years 2001-2006.

\subsubsection{Mean FL at Age}

Comparisons of mean FL at age were possible for 3 groups (Table 23). There was no significant difference in mean FL between hatchery- and natural-origin age 3 returns ( $\mathrm{t}$ ratio $=-0.8826, \mathrm{P}>|\mathrm{t}|=0.4032)$. Mean FL at age 4 were significantly different between hatchery- and natural-origin males $(\mathrm{t}$ ratio $=-4.14538, \mathrm{P}>|\mathrm{t}|=0.0003)$ and females $(\mathrm{t}$ ratio $=-2.2488, \mathrm{P}>|\mathrm{t}|=0.0290)$.

Table 23. FL at age summary by sex for natural- and hatchery-origin Catherine Creek spring Chinook salmon collected at the trap or on spawning ground surveys, 2007.

\begin{tabular}{ccccccc}
\hline Age & Sex & Origin & Mean FL $(\mathrm{mm})$ & SE & Range & N \\
\hline 3 & M & Natural & 468.2 & 28.1 & $423-579$ & 5 \\
3 & M & Hatchery & 500.6 & 23.6 & $415-548$ & 5 \\
4 & M & Natural & 692.4 & 35.6 & $585-821$ & 12 \\
4 & M & Hatchery & 776.7 & 11.4 & $670-844$ & 15 \\
4 & F & Natural & 693.9 & 14.1 & $610-830$ & 14 \\
4 & F & Hatchery & 722.9 & 5.9 & $650-793$ & 37 \\
5 & M & Natural & 865.8 & 18.7 & $791-962$ & 8 \\
5 & M & Hatchery & 845 & & & 1 \\
5 & F & Natural & 846.2 & 21.8 & $765-944$ & 9 \\
\hline
\end{tabular}




\subsubsection{Environmental Data}

Mean daily water temperatures in 2007 at the Catherine Creek acclimation facility during the period 26 March-11 April ranged from 5.0-8.8 ${ }^{\circ} \mathrm{C}$ (Figure 16). Daily fluctuations ranged from $0.9-5.8^{\circ} \mathrm{C}$ during the release period.

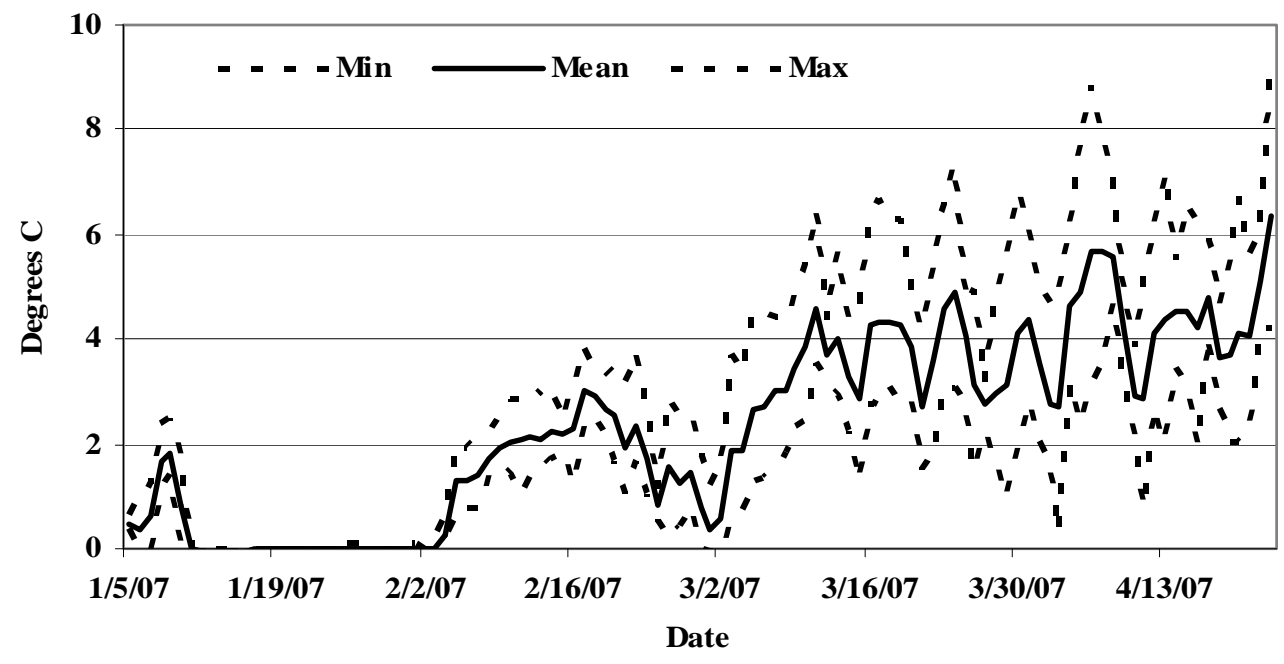

Figure 16. Daily minimum, mean, and maximum water temperatures at the Catherine Creek acclimation site, 2007.

The highest daily water temperature recorded at the Catherine Creek adult trap site in 2007 was $25.9^{\circ} \mathrm{C}$ on 23 July, and the maximum daily fluctuation was $9.5^{\circ} \mathrm{C}$ on 1 August (Figure 17). 


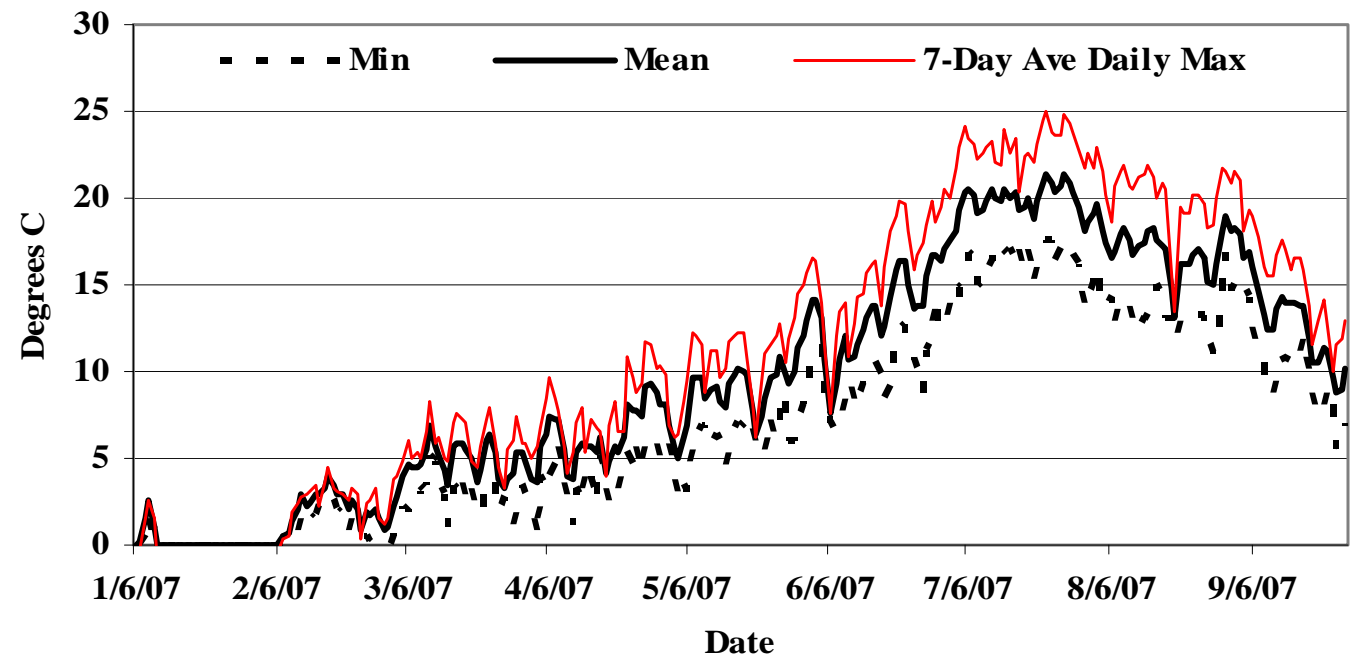

Figure 17. Daily minimum, mean, and maximum water temperatures at the Catherine Creek adult site, 2007.

The highest daily water temperature recorded at the North Fork Catherine Creek site was $20.2^{\circ} \mathrm{C}$ on $27-28$ July (Figure 18). The maximum daily fluctuation of $8.4^{\circ} \mathrm{C}$ occurred on 3 dates in August.

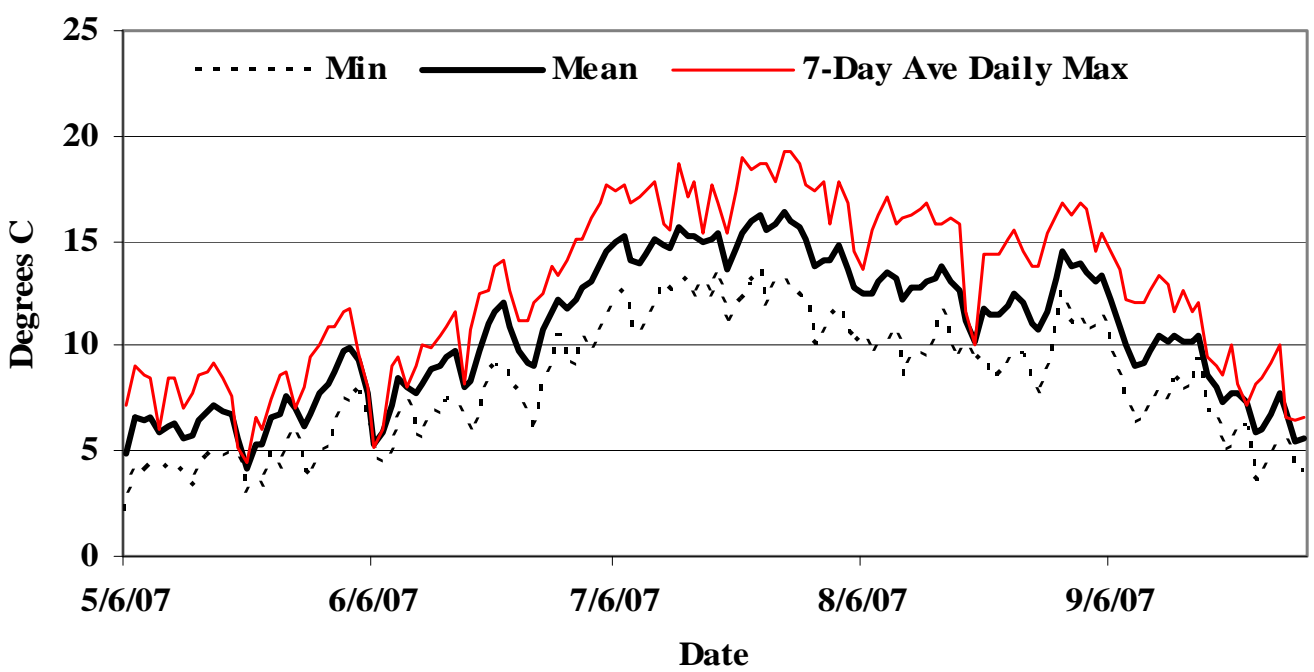

Figure 18. Daily minimum, mean, and 7-day average maximum water temperatures at the North Fork Catherine Creek site, 2007 (data courtesy Wallowa-Whitman National Forest). 
North Fork Catherine Creek stream flow data were not available at the writing of this report. The pattern of snowfall and spring thawing left flows below normal in the spring and flows remained low throughout much of the summer. Flow for the Grande Ronde River at the Troy, Oregon gauge peaked about 2 months earlier than normal, and the peak flow was about $66 \%$ of the long-term average (Figure 19). The water year (WY) goes from 1 October to 30 September of the following year.

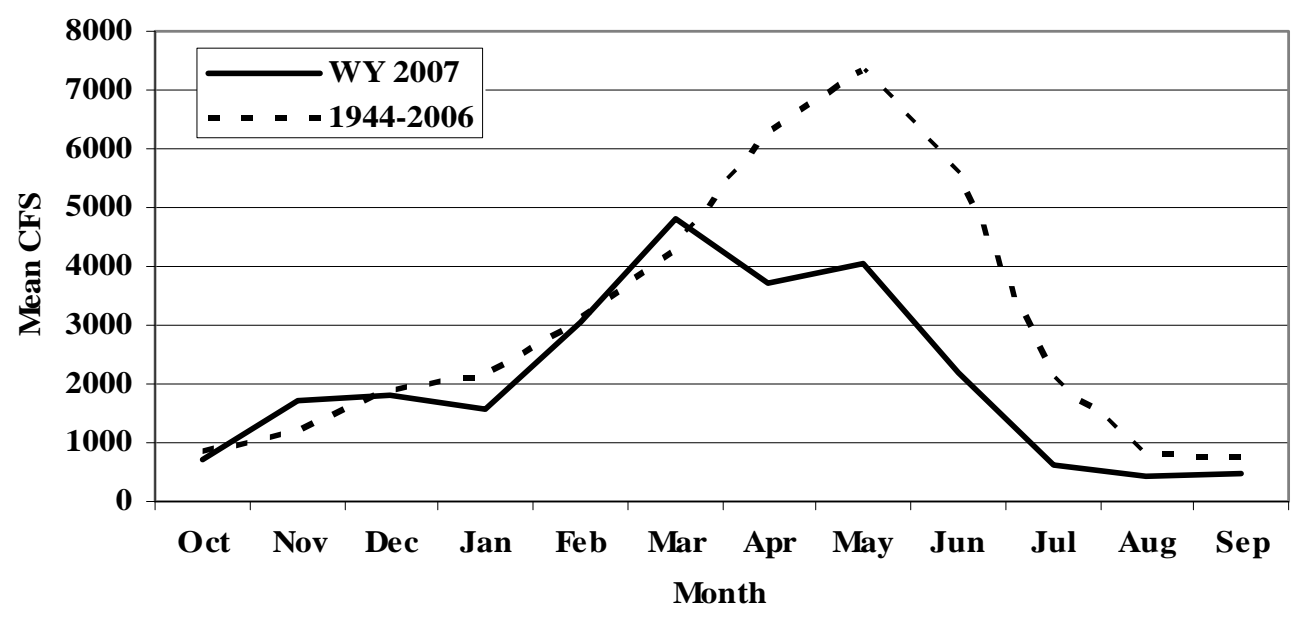

Figure 19. Mean daily flows (CFS) recorded at the USGS gauging station on the Grande Ronde River near Troy, OR, WY 2007 and WY 1944-2006.

\subsubsection{Upper Grande Ronde River Juvenile Chinook Salmon}

\subsubsection{Size at Release}

There were significant differences in mean FL at PIT-tagging between captive and conventional broodstock progeny, and hatchery- and natural-origin juveniles (Table 24), but none between captive and conventional broodstock progeny sampled at pretransfer in February 2007 (Table 25). There was a significant difference in mean FL at the acclimation facility between prevolitional and preforceout conventional broodstock progeny, but not for captive broodstock progeny. The mean FL of prevolitional and preforceout captive and conventional broodstock progeny were 5.0-9.6 greater than captive and conventional broodstock progeny sampled at pretransfer in February 2007. Captive and conventional broodstock groups differed by only 0.2-1.2 mm between PITtagging in October 2006 and pretransfer sampling in February 2007. Using all hatcheryorigin fish at pretransfer sampling, the mean FL was 7.4 greater than at PIT-tagging. Mean weights at PIT-tagging showed similar patterns as for mean FL at PIT-tagging 
(Table 26). Mean weights were very similar for captive and conventional broodstock progeny groups sampled at pretransfer (Table 27). Captive broodstock prevolitional release fish were $0.6 \mathrm{~g}$ heavier than forceout fish of the same group, but conventional broodstock prevolitional release fish were $0.5 \mathrm{~g}$ lighter than forceout.

Table 24. FL at PIT-tagging for brood year (BY) 2005 spring Chinook salmon by rearing and release type from the upper Grande Ronde River (CTUIR data and ODFW, unpublished data).

\begin{tabular}{|c|c|c|c|c|c|c|c|}
\hline \multirow[b]{2}{*}{ Rearing $^{\mathrm{a}}$} & \multirow[b]{2}{*}{ Release $^{\mathrm{b}}$} & \multicolumn{6}{|c|}{$\mathrm{FL}(\mathrm{mm})$} \\
\hline & & Mean & $\mathrm{SE}$ & Range & $\mathrm{N}$ & $t$ ratio & $P>|t|$ \\
\hline $\mathrm{H}$ & $\mathrm{CaV}$ & 115.3 & 1.8 & $97-154$ & 30 & 1.427683 & 0.1582 \\
\hline $\mathrm{H}$ & $\mathrm{CaFO}$ & 112.3 & 1.2 & $95-126$ & 38 & & \\
\hline $\mathrm{H}$ & $\mathrm{CoV}$ & 105.4 & 1.0 & 89-118 & 40 & 0.116177 & 0.9077 \\
\hline $\mathrm{H}$ & CoFO & 105.3 & 0.6 & $90-123$ & 97 & & \\
\hline $\mathrm{H}$ & $\mathrm{Ca}$ & 113.5 & 0.9 & $95-154$ & 81 & -8.21615 & $<0.0001$ \\
\hline $\mathrm{H}$ & Co & 105.2 & 0.5 & 86-123 & 166 & & \\
\hline $\mathrm{H}$ & $\mathrm{Ca}$ & 113.5 & 0.9 & $95-154$ & 81 & -21.3388 & $<0.0001$ \\
\hline $\mathrm{N}$ & Early & 82.5 & 1.0 & $68-98$ & 49 & & \\
\hline $\mathrm{H}$ & Co & 105.2 & 0.5 & 86-123 & 166 & -19.3577 & $<0.0001$ \\
\hline $\mathrm{N}$ & Late & 85.0 & 1.0 & 73-100 & 49 & & \\
\hline $\mathrm{H}$ & All & 106.4 & 0.5 & 84-154 & 328 & & \\
\hline $\mathrm{N}$ & All & 83.8 & 0.7 & 64-100 & 124 & & \\
\hline
\end{tabular}


Table 25. FL by release or tag group for brood year (BY) 2005 hatchery-origin spring Chinook salmon from the upper Grande Ronde River (CTUIR data and ODFW, unpublished data).

\begin{tabular}{|c|c|c|c|c|c|c|}
\hline \multirow[b]{2}{*}{ Group $^{a}$} & \multicolumn{6}{|c|}{ FL (mm) } \\
\hline & Mean & $\mathrm{SE}$ & Range & $\mathrm{N}$ & $t$ ratio & $P>|t|$ \\
\hline CaPT & 113.3 & 1.3 & $85-128$ & 50 & 0.511544 & 0.6095 \\
\hline CoPT & 114.0 & 0.7 & 85-130 & 150 & & \\
\hline PT (all) & 113.8 & 0.6 & 85-130 & 200 & & \\
\hline PIT & 106.4 & 0.5 & 84-154 & 328 & & \\
\hline CaPV & 121.4 & 0.7 & 96-137 & 120 & 2.621035 & 0.0094 \\
\hline CaPFO & 118.3 & 0.9 & 98-148 & 100 & & \\
\hline Ca PIT & 113.5 & 0.9 & $95-154$ & 81 & & \\
\hline CoPV & 122.2 & 0.5 & $102-142$ & 199 & -1.91907 & 0.0557 \\
\hline CoPFO & 123.6 & 0.6 & $100-141$ & 200 & & \\
\hline Co PIT & 105.2 & 0.5 & 86-123 & 166 & & \\
\hline
\end{tabular}


Table 26. Weight at PIT-tagging for BY 2005 spring Chinook salmon by rearing method and release type from the upper Grande Ronde River (CTUIR data and ODFW, unpublished data).

\begin{tabular}{cccccc}
\hline & & \multicolumn{4}{c}{ Weight $(\mathrm{g})$} \\
\cline { 3 - 6 } Rearing & Release & Mean & SE & Range & $\mathrm{N}$ \\
\hline $\mathrm{H}$ & $\mathrm{CaV}$ & 17.7 & 0.6 & $13.2-21.7$ & 18 \\
$\mathrm{H}$ & $\mathrm{CaFO}$ & 16.8 & 0.7 & $9.6-23.6$ & 28 \\
& & & & & \\
$\mathrm{H}$ & $\mathrm{CoV}$ & 14.3 & 0.5 & $10.6-19.3$ & 28 \\
$\mathrm{H}$ & $\mathrm{CoFO}$ & 14.1 & 0.3 & $9.0-20.2$ & 55 \\
& & & & & \\
$\mathrm{H}$ & $\mathrm{Ca}$ & 17.4 & 0.5 & $9.3-24.0$ & 53 \\
$\mathrm{H}$ & $\mathrm{Co}$ & 14.7 & 0.2 & $7.4-20.2$ & 104 \\
& & & & & \\
$\mathrm{H}$ & $\mathrm{Ca}$ & 17.4 & 0.5 & $9.3-24.0$ & 53 \\
$\mathrm{~N}$ & Early & 5.3 & 0.2 & $2.7-9.1$ & 49 \\
& & & & & \\
$\mathrm{H}$ & Co & 14.1 & 0.2 & $7.4-20.2$ & 104 \\
$\mathrm{~N}$ & Late & 6.2 & 0.2 & $3.8-10.8$ & 49 \\
$\mathrm{H}$ & & & & & \\
$\mathrm{N}$ & All & 14.3 & 0.2 & $6.2-24.0$ & 213 \\
\hline
\end{tabular}


Table 27. Weight by release or tag group for brood year (BY) 2005 hatchery-origin spring Chinook salmon from the upper Grande Ronde River (CTUIR data and ODFW, unpublished data).

\begin{tabular}{ccccc}
\hline & \multicolumn{4}{c}{ Weight (g) } \\
\cline { 2 - 5 } Group & Mean & SE & Range & N \\
\hline CaPT & 19.2 & 0.7 & $8.7-29.2$ & 50 \\
CoPT & 19.3 & 0.4 & $5.4-35.2$ & 150 \\
PT All & 19.2 & 0.3 & $5.4-35.2$ & 200 \\
PIT & 14.3 & 0.2 & $6.2-24.0$ & 213 \\
& & & & \\
CaPV & 20.3 & 0.4 & $8.7-31.2$ & 120 \\
CaPFO & 19.7 & 0.6 & $10.1-37.9$ & 100 \\
Ca PIT & 17.4 & 0.5 & $9.3-24.0$ & 53 \\
& & & & \\
CoPV & 21.5 & 0.3 & $11.5-35.7$ & 199 \\
CoPFO & 22.0 & 0.3 & $10.4-34.2$ & 200 \\
Co PIT & 14.7 & 0.2 & $7.4-20.2$ & 104 \\
\hline
\end{tabular}

Mean K factors at PIT-tagging were significantly different between captive broodstock volitionally-released and forceout, captive broodstock and conventional broodstock progeny, and hatchery- and natural-origin (Table 28). There were also significant differences between prevolitional and preforceout groups for both captive and conventional broodstock progeny. Mean K factors for captive and conventional broodstock progeny at pretransfer sampling were 0.1-0.15 greater than at PIT-tagging (Table 29). 
Table 28. K factor at PIT-tagging summary for BY 2005 spring Chinook salmon by rearing method and release type from the upper Grande Ronde River (CTUIR.data and ODFW, unpublished data).

\begin{tabular}{cccccccc}
\hline & & \multicolumn{7}{c}{ K factor } \\
\cline { 3 - 8 } Rearing & Release & Mean & SE & Range & $\mathrm{N}$ & $t$ ratio & $\mathrm{P}>|\mathrm{t}|$ \\
\hline $\mathrm{H}$ & $\mathrm{CaV}$ & 1.16 & 0.02 & $1.06-1.30$ & 18 & 2.19877 & 0.0332 \\
$\mathrm{H}$ & $\mathrm{CaFO}$ & 1.12 & 0.01 & $0.97-1.22$ & 28 & & \\
$\mathrm{H}$ & $\mathrm{CoV}$ & 1.18 & 0.01 & $1.05-1.30$ & 28 & 0.719161 & 0.4741 \\
$\mathrm{H}$ & $\mathrm{CoFO}$ & 1.17 & 0.01 & $0.94-1.37$ & 55 & & \\
& & & & & & & \\
$\mathrm{H}$ & $\mathrm{Ca}$ & 1.13 & 0.01 & $0.96-1.35$ & 53 & 2.827293 & 0.0054 \\
$\mathrm{H}$ & $\mathrm{Co}$ & 1.17 & 0.01 & $0.89-1.37$ & 104 & & \\
& & & & & & & \\
$\mathrm{H}$ & Ca & 1.13 & 0.01 & $0.96-1.35$ & 53 & -10.8151 & $<0.0001$ \\
$\mathrm{~N}$ & Early & 0.93 & 0.02 & $0.69-1.47$ & 49 & & \\
$\mathrm{H}$ & Co & 1.17 & 0.01 & $0.89-1.37$ & 104 & -10.1815 & $<0.0001$ \\
$\mathrm{~N}$ & Late & 1.00 & 0.02 & $0.74-1.39$ & 49 & & \\
$\mathrm{H}$ & All & 1.15 & 0.01 & $0.89-1.37$ & 213 & -12.7387 & $<0.0001$ \\
$\mathrm{~N}$ & All & 0.97 & 0.02 & $0.69-2.42$ & 121 & & \\
\hline
\end{tabular}


Table 29. K factor by release or tag group for brood year (BY) 2005 hatchery-origin spring Chinook salmon from the upper Grande Ronde River (CTUIR data and ODFW, unpublished data).

\begin{tabular}{|c|c|c|c|c|c|c|}
\hline \multirow[b]{2}{*}{ Group } & \multicolumn{6}{|c|}{ K factor } \\
\hline & Mean & $\mathrm{SE}$ & Range & $\mathrm{N}$ & $t$ ratio & $P>|t|$ \\
\hline CaPT & 1.28 & 0.02 & $1.02-1.69$ & 50 & -0.74936 & 0.4545 \\
\hline CoPT & 1.27 & 0.01 & 0.79-1.76 & 150 & & \\
\hline PT (all) & 1.28 & 0.01 & $0.79-1.76$ & 200 & & \\
\hline PIT & 1.15 & 0.01 & 0.89-1.37 & 213 & & \\
\hline $\mathrm{CaPV}$ & 1.12 & 0.01 & $0.95-1.28$ & 120 & 2.621035 & 0.0094 \\
\hline CaPFO & 1.16 & 0.01 & $0.91-1.82$ & 100 & & \\
\hline Ca PIT & 1.13 & 0.01 & $0.96-1.35$ & 53 & & \\
\hline CoPV & 1.17 & 0.01 & $0.75-1.96$ & 199 & 3.473067 & 0.0006 \\
\hline CoPFO & 1.15 & 0.01 & $0.72-1.53$ & 200 & & \\
\hline Co PIT & 1.17 & 0.01 & 0.89-1.37 & 104 & & \\
\hline
\end{tabular}

\subsubsection{Migration Timing}

There were significant differences in arrival date at Lower Granite Dam for all groups compared (Table 30). Median arrival dates of volitionally-released juveniles were earlier than forceouts, captive broodstock progeny were earlier than conventional broodstock progeny and hatchery-origin were earlier than natural-origin. Median arrival dates, first arrivals, and last arrivals differed little between hatchery-origin groups, but there were substantial differences between the hatchery-origin and natural-origin. 
Table 30. Arrival dates in 2007 at Lower Granite Dam of PIT-tagged BY 2005 spring Chinook salmon by rearing method and release type from the upper Grande Ronde River.

\begin{tabular}{|c|c|c|c|c|c|c|c|c|}
\hline Rearing & Release & $\mathrm{N}^{*}$ & $\mathrm{~N}_{\exp } * *$ & Median & Earliest & Latest & $Z$ value & $\mathrm{P}>|\mathrm{Z}|$ \\
\hline $\mathrm{H}$ & $\mathrm{CaV}$ & 20 & 35 & 1 May & 14 April & 13 Мау & 2.63 & 0.0086 \\
\hline $\mathrm{H}$ & $\mathrm{CaFO}$ & 17 & 27 & 5 May & 20 April & 16 May & & \\
\hline $\mathrm{H}$ & $\mathrm{CoV}$ & 78 & 108 & 2 May & 16 April & 15 Мау & -5.91 & $<0.0001$ \\
\hline $\mathrm{H}$ & $\mathrm{CoFO}$ & 142 & 186 & 4 May & 27 April & 18 May & & \\
\hline $\mathrm{H}$ & $\mathrm{Ca}$ & 42 & 65 & 2 May & 14 April & 16 Мау & 1.51708 & 0.1292 \\
\hline $\mathrm{N}$ & Early & 5 & 9 & 13 May & 13 April & 19 May & & \\
\hline $\mathrm{H}$ & Co & 221 & 286 & 3 May & 16 April & 18 Мау & 6.33729 & $<0.0001$ \\
\hline $\mathrm{N}$ & Late & 9 & 15 & 18 May & 13 May & 22 May & & \\
\hline $\mathrm{H}$ & All & 302 & 390 & 3 May & 14 April & 18 Мау & 7.18031 & $<0.0001$ \\
\hline $\mathrm{N}$ & All & 22 & 35 & 14 May & 13 April & 11 June & & \\
\hline
\end{tabular}

Frequency distributions of arrival dates for both hatchery- and natural-origin outmigrants appeared bimodal, but the distribution for natural-origin outmigrants was later than for hatchery-origin (Figures 20-21). Median arrival dates for both groups occurred at peak or near-peak flows (Figure 22). 


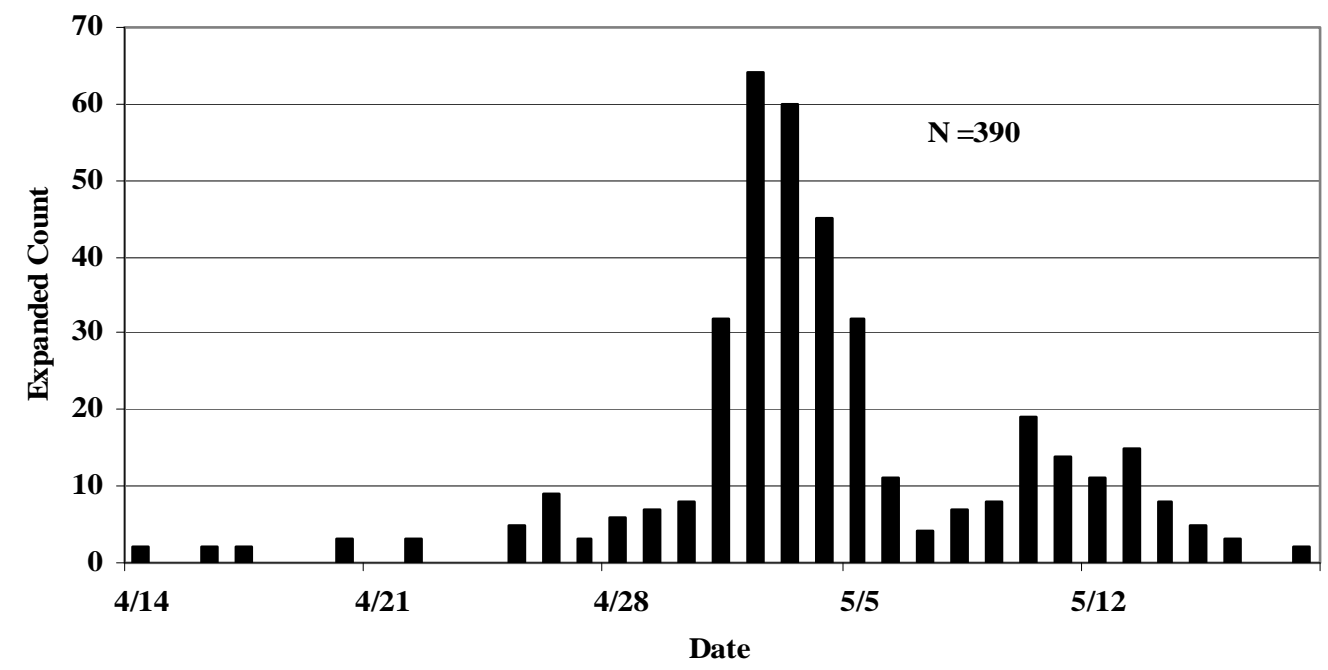

Figure 20. Frequency distribution of arrival dates (expanded for spill) at Lower Granite Dam for upper Grande Ronde River hatchery-origin spring Chinook salmon, BY 2005.

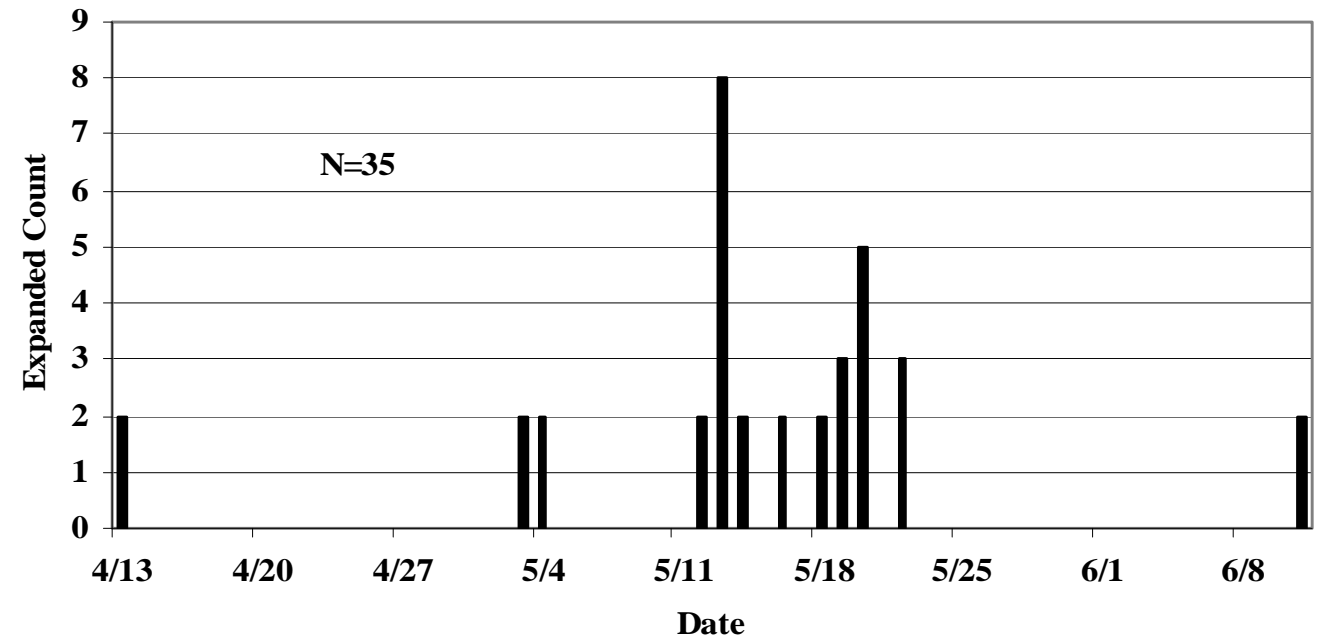

Figure 21. Frequency distribution of arrival dates (expanded for spill) at Lower Granite Dam for upper Grande Ronde River natural-origin spring Chinook salmon (spring release group), BY 2005. 


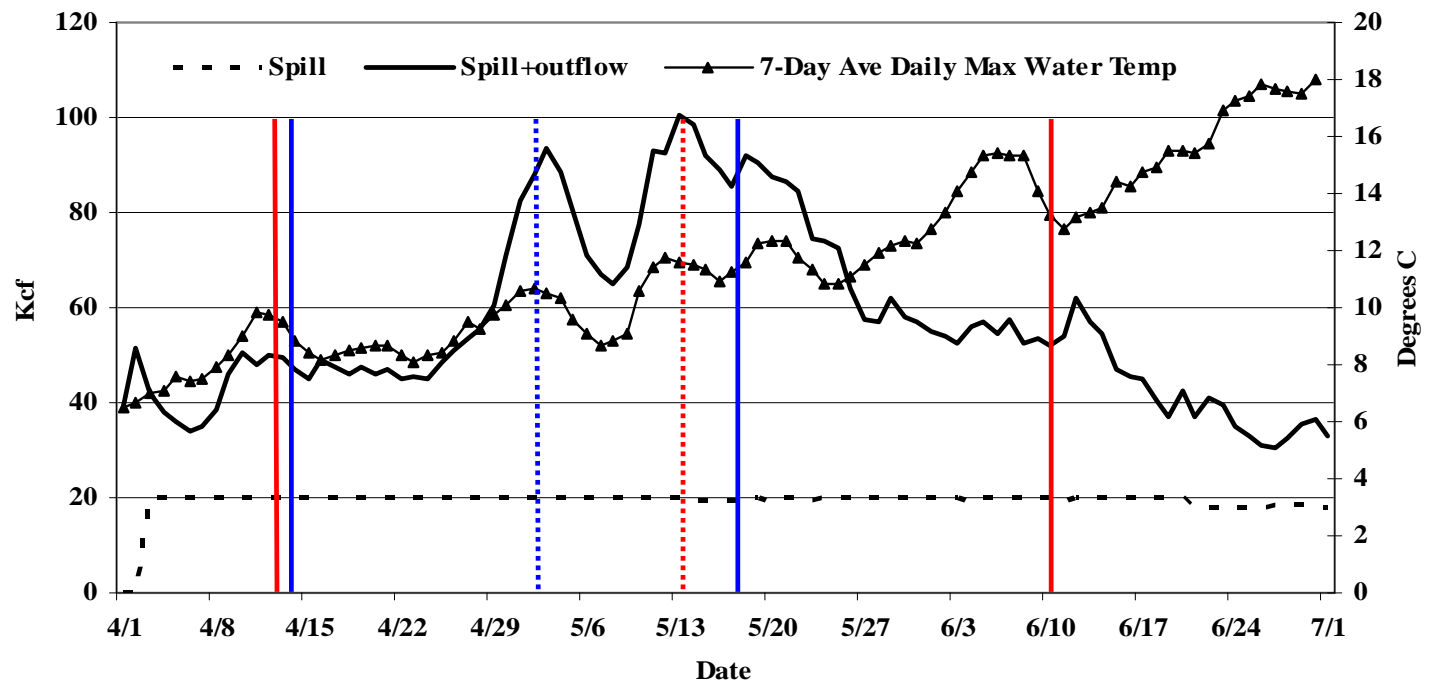

Figure 22. First and last (solid vertical lines) detection dates and median detection dates (dashed vertical lines) at Lower Granite Dam (expanded for spill) for upper Grande Ronde River BY 2005 natural- (blue lines) and hatchery-origin (red lines) juvenile spring Chinook salmon.

There appeared to be significant differences in travel time to Lower Granite between volitionally-released and forceout groups, captive broodstock and conventional broodstock progeny groups, and hatchery- and natural-origin groups (Table 31). 
Table 31. Harmonic means (d) of travel time to Lower Granite Dam of PIT-tagged BY 2005 spring Chinook salmon by rearing and release type from the upper Grande Ronde River.

\begin{tabular}{ccccc}
\hline & & \multicolumn{3}{c}{ Harmonic } \\
Rearing & Release & $\mathrm{N}$ & Mean & $\mathrm{SE}$ \\
\hline $\mathrm{H}$ & $\mathrm{CaV}$ & 20 & 40.120 & 1.876 \\
$\mathrm{H}$ & $\mathrm{CaFO}$ & 17 & 46.149 & 1.721 \\
& & & & \\
$\mathrm{H}$ & $\mathrm{CoV}$ & 64 & 29.725 & 0.703 \\
$\mathrm{H}$ & $\mathrm{CoFO}$ & 23 & 33.022 & 0.332 \\
& & & & \\
$\mathrm{~N}$ & Early & 5 & 40.626 & 9.437 \\
$\mathrm{H}$ & $\mathrm{Ca}$ & 52 & 43.264 & 1.308 \\
& & & & \\
$\mathrm{~N}$ & Late & 9 & 39.031 & 1.923 \\
$\mathrm{H}$ & $\mathrm{Co}$ & 220 & 31.930 & 0.325 \\
& & & & \\
$\mathrm{H}$ & All & 302 & 33.166 & 0.344 \\
$\mathrm{~N}$ & All & 22 & 42.216 & 2.618 \\
& & & & \\
\hline
\end{tabular}

\subsubsection{Survival}

Survival rates for volitionally-released and forceout groups were similar (Table 32), but was higher for conventional broodstock compared to captive broodstock progeny and for hatchery- compared to natural-origin outmigrants. Significant differences in capture probabilities were probably present for the captive broodstock-conventional broodstock and hatchery-natural group comparisons. 
Table 32. Survival to and capture probabilities $\left(\mathrm{C}_{\mathrm{p}}\right)$ of BY 2005 spring Chinook salmon at Lower Granite Dam by rearing method and release type from the upper Grande Ronde River.

\begin{tabular}{ccccccc}
\hline Rearing & Release & $\mathrm{N}$ & Survival & $\mathrm{SE}$ & $\mathrm{C}_{\mathrm{p}}$ & $\mathrm{SE}$ \\
\hline $\mathrm{H}$ & $\mathrm{CaV}$ & 185 & 0.3270 & 0.0558 & 0.3306 & 0.0750 \\
$\mathrm{H}$ & $\mathrm{CaFO}$ & 231 & 0.3333 & 0.0614 & 0.2208 & 0.0589 \\
& & & & & & \\
$\mathrm{H}$ & $\mathrm{CoV}$ & 310 & 0.5602 & 0.0497 & 0.3697 & 0.0456 \\
$\mathrm{H}$ & $\mathrm{CoFO}$ & 693 & 0.5563 & 0.0353 & 0.3191 & 0.0292 \\
& & & & & & \\
$\mathrm{~N}$ & Early & 49 & 0.2109 & 0.0716 & 0.4839 & 0.1824 \\
$\mathrm{H}$ & $\mathrm{Ca}$ & 496 & 0.3528 & 0.0434 & 0.2400 & 0.0412 \\
& & & & & & \\
$\mathrm{~N}$ & Late & 49 & 0.3469 & 0.0680 & 0.5294 & 0.1211 \\
$\mathrm{H}$ & $\mathrm{Co}$ & 1,283 & 0.5378 & 0.0258 & 0.3191 & 0.0219 \\
& & & & & & \\
$\mathrm{H}$ & All & 1,985 & 0.4956 & 0.0210 & 0.3084 & 0.0184 \\
$\mathrm{~N}$ & All & 124 & 0.3172 & 0.0516 & 0.5593 & 0.0955 \\
\hline
\end{tabular}

\subsubsection{Upper Grande Ronde River Adult Spring Chinook Salmon}

\subsubsection{Harvest}

CWT recoveries of upper Grande Ronde River stock BY 2002 returns were for only one tag code and none were recovered in sport or commercial fisheries (Table 33). 
Table 33. Estimated tag recoveries of BY 2002 spring Chinook salmon in the Columbia River in 2005-2006 that were released from the upper Grande Ronde River acclimation facility.

\begin{tabular}{cccc}
\hline Tag code & Run Year & Recovery Location & Estimated Number \\
\hline 93833 & 2005 & UGR SGS & 1 \\
93833 & 2005 & UGR Trap & 5 \\
& Run Year 2005 Total & & 6 \\
93833 & 2006 & UGR SGS & 4 \\
93833 & 2006 & UGR trap & 128.26 \\
93833 & 2006 & LKG SGS & 4 \\
93833 & 2006 & NWTR 026-00 & 4.45 \\
93833 & 2006 & Round Butte Trap & 1.02 \\
& Run Year 2005 Total & UGR trap & 141.73 \\
93833 & 2007 & & 8.32 \\
& Run Year 2007 Total & & 8.32 \\
\hline
\end{tabular}

\subsubsection{Trap Catch}

The upper Grande Ronde River trap was operated from 3 March-13 July 2007. Naturalorigin adults were passed above the trap to spawn or transported to Lookingglass Hatchery to be held for use as conventional broodstock. Disposition of hatchery-origin adults included trap mortalities, passing above the trap to spawn, and transporting to Lookingglass Hatchery to use for conventional broodstock. Only conventional broodstock progeny were used for spawning at Lookingglass Hatchery.

A total of 73 adults (39 females, 34 males) were collected from 18 May-27 June; none were confirmed as strays by visual marks. A $617 \mathrm{~mm}$ FL natural-origin female collected on 15 June had a radio tag.

Natural-origin adults totaled 33 (17 females, 16 males). Males were collected from 21 May-18 June and females from 21 May-27 June. Seventeen adults (9 females, 8 males) were transported to Lookingglass Hatchery and 16 (8 females, 8 males) were passed above the trap to spawn naturally.

Hatchery-origin (all conventional broodstock progeny) adults totaled 40 (22 females, 18 males). Males were collected from 25 May-25 June and females from 18 May-20 June. Three males from 457-467 mm were released above the trap to spawn naturally and one $816 \mathrm{~mm}$ female was a trap mortality; the remainder were transported to Lookingglass Hatchery to use as conventional broodstock. 
Peak catches of both hatchery- and natural-origin adults occurred the week of 28 May. The cumulative frequency distributions for arrival timing at the trap between hatcheryand natural-origin adults were similar (Figure 23) and the Kolmogorov-Smirnov test for a difference in arrival date distributions was not significant (2-tailed K-S statistic $=0.04$, $\mathrm{P}=1.0000)$.

Water temperature data for the upper Grande Ronde River adult trap site were not collected for the 2007 spring Chinook salmon trapping season and 2007 stream flow data for the site near Clear Creek were not available when this report was written.

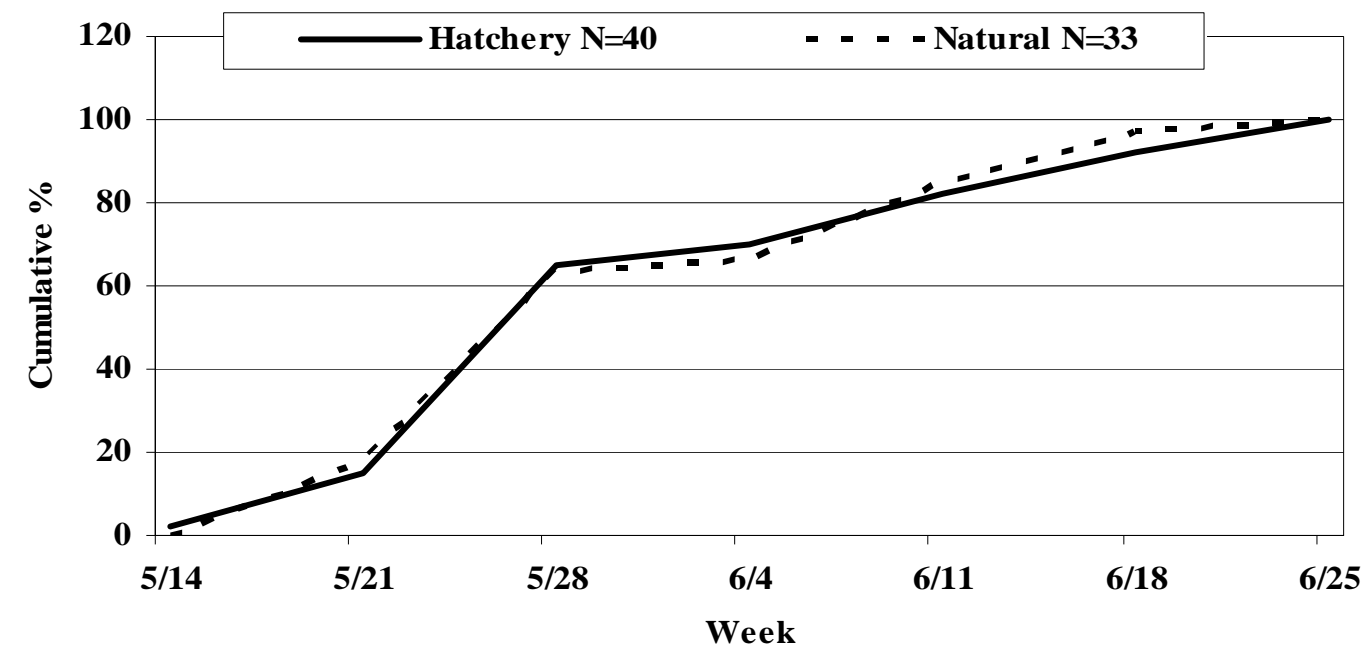

Figure 23. Cumulative percentages of natural- and hatchery-origin spring Chinook salmon caught by week of the year and age group at the upper Grande Ronde River trap, 2007.

Few adults were $<60 \mathrm{~cm}$, most were in the $66-80 \mathrm{~cm}$ groups, and a substantial number were $>80 \mathrm{~cm}$ (Figure 24). Natural-origin adults were more dispersed among the FL groups than hatchery-origin (Figures 25-26). There was no difference in FL (cm) distributions between hatchery- and natural-origin adults (2-tailed K-S statistic $=0.20$, $\mathrm{P}=0.4405)$. 


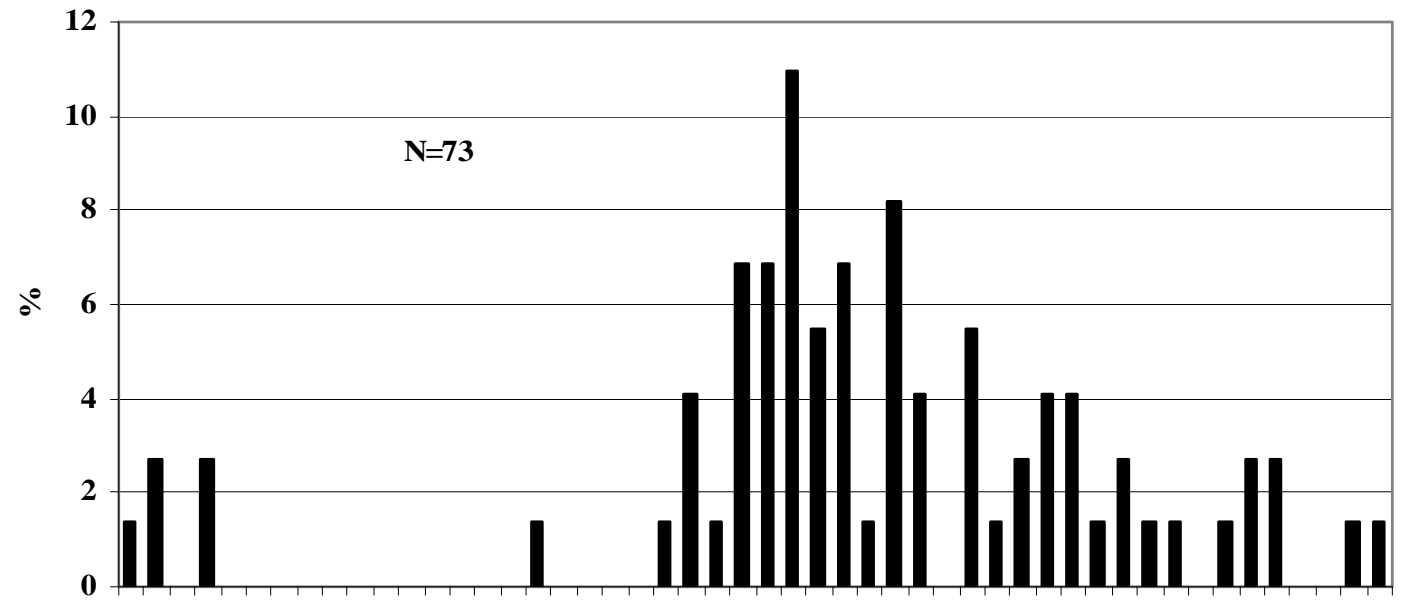

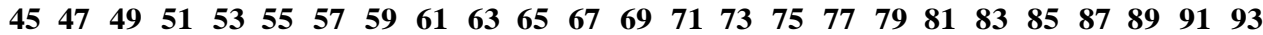

FL (cm)

Figure 24. FL distribution of all spring Chinook salmon caught at the upper Grande Ronde River trap, 2007.

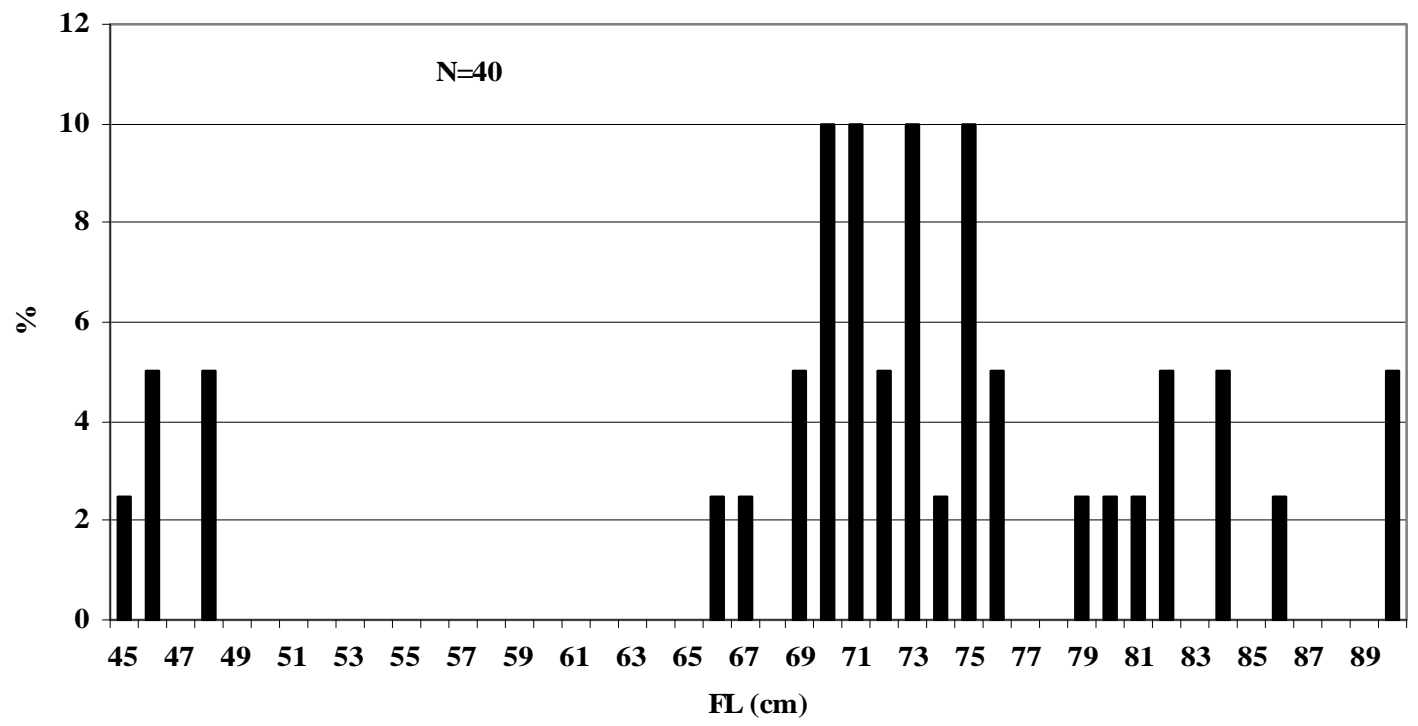

Figure 25. FL distribution of hatchery-origin (conventional broodstock progeny) spring Chinook salmon caught at the upper Grande Ronde River trap, 2007. 


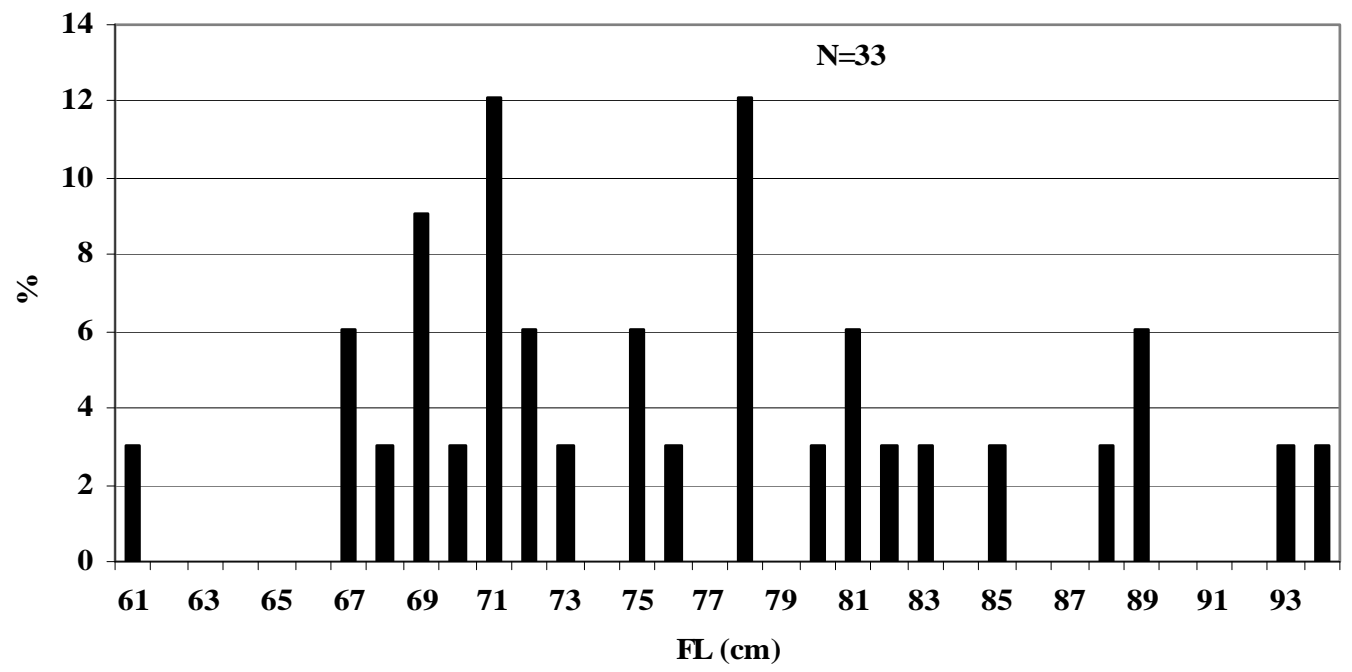

Figure 26. FL distribution of natural-origin spring Chinook salmon caught at the upper Grande Ronde River trap, 2007.

\subsubsection{Conventional spawning}

Conventional broodstock included 14 natural- (6 females, 8 males) and 46 hatcheryorigin (26 females, 20 males). Mortalities at Lookingglass Hatchery included 13 hatchery-origin males and 2 natural-origin females. Spawn dates in 2007 for naturalorigin females were (number in parentheses) 6 September (2), 11 (September (2), and 17 September (2). Spawn dates for hatchery-origin females were 15 August (1), 6 September (4), 11 September (13), and 17 September (8). Spawn timing was similar between hatchery- and natural-origin females (Wilcoxon test, $\mathrm{Z}=-0.23418, \mathrm{P}>|\mathrm{Z}|=$ 0.8148).

Hatchery-origin females were more equally distributed among the FL groups than natural-origin (Figure 27). Mean FL of spawned hatchery-origin males was greater than natural-origin, but the reverse occurred for females (Table 34).

Mean weights differed by $0.1 \mathrm{~kg}$ between rear types (Table 35). The length-weight regression for natural-origin females fit much better than for hatchery-origin (Table 36). Predicted weights for hatchery- and natural-origin females $750 \mathrm{~mm}$ FL were $4.1 \mathrm{~kg}$ for hatchery-origin and $4.2 \mathrm{~kg}$ for natural-origin using equations in Table 36. 


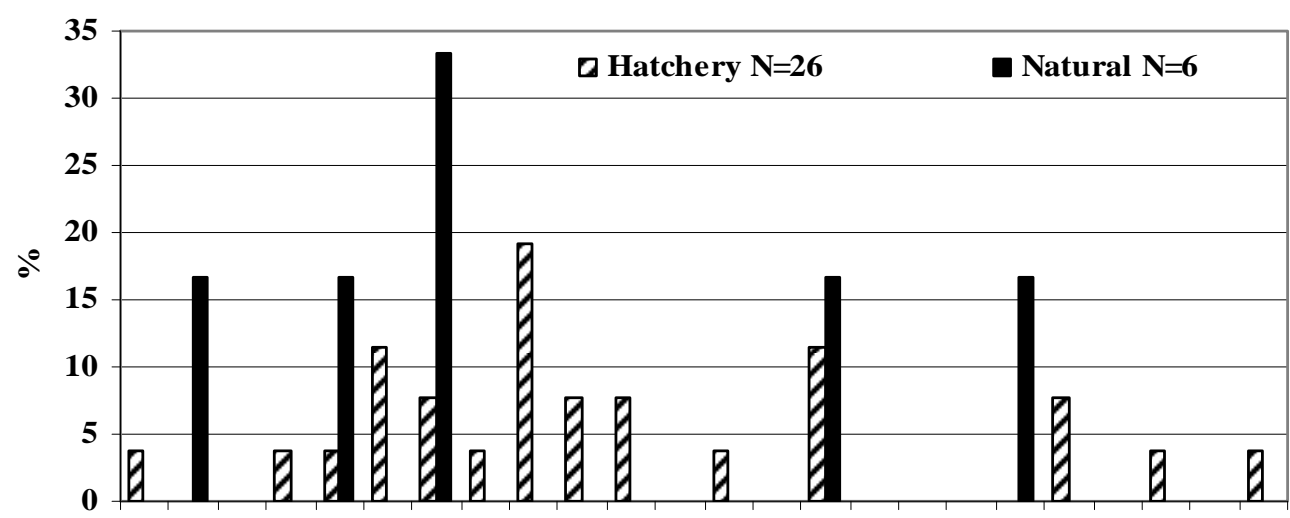

$\begin{array}{llllllllllllllllllllllll}66 & 67 & 68 & 69 & 70 & 71 & 72 & 73 & 74 & 75 & 76 & 77 & 78 & 79 & 80 & 81 & 82 & 83 & 84 & 85 & 86 & 87 & 88 & 89\end{array}$

FL (cm)

Figure 27. FL distribution for female upper Grande Ronde River conventional broodstock (CWT, no ad clip), 2007.

Table 34. FL summary for upper Grande Ronde River conventional broodstock spawned in 2007.

\begin{tabular}{cccccc}
\hline Origin & Sex & Mean FL $(\mathrm{mm})$ & SE & Range & N \\
\hline Hatchery & M & 697.9 & 34.4 & $456-935$ & 20 \\
Natural & M & 786.4 & 26.5 & $715-925$ & 8 \\
Hatchery & F & 760.4 & 11.2 & $666-892$ & 26 \\
Natural & F & 745.2 & 25.3 & $677-840$ & 6 \\
\hline
\end{tabular}

Table 35. Weight summary for upper Grande Ronde River conventional broodstock females spawned in 2007.

\begin{tabular}{cccccc}
\hline Origin & Sex & Mean Wgt $(\mathrm{kg})$ & SE & Range & N \\
\hline Hatchery & F & 4.3 & 0.2 & $2.9-6.9$ & 26 \\
Natural & F & 4.2 & 0.5 & $3.0-6.3$ & 6 \\
\hline
\end{tabular}

Table 36. Length-weight (log) regression summary for female upper Grande Ronde River conventional broodstock spawned in 2007.

\begin{tabular}{ccccc}
\hline Origin & Slope & Intercept & Adj. $\mathrm{R}^{2}$ & $\mathrm{~N}$ \\
\hline Hatchery & 2.2244184 & -5.786939 & 0.507411 & 26 \\
Natural & 3.5541564 & -9.598673 & 0.984034 & 6 \\
\hline
\end{tabular}


Mean fecundity differed by 17 between hatchery- and natural-origin (Table 37). The two-sample t-test for difference in fecundity between natural- and hatchery-origin females was not significant $(\mathrm{t}$ ratio $=-0.69885, \mathrm{P}>|\mathrm{t}|=0.4902)$.

Mean egg weights were $0.23 \mathrm{~g}$ (range $0.16-0.27$ ) for hatchery-origin and $0.22 \mathrm{~g}$ (range $(0.19-0.26)$ for natural-origin and not significantly different $(\mathrm{t}$ ratio $=-0.65861, \mathrm{P}>|\mathrm{t}|=$ $0.51532)$.

Table 37. Fecundity (eggs/female) summary for upper Grande Ronde River conventional broodstock spawned in 2007.

\begin{tabular}{ccccccc}
\hline Origin & Mean & SE & Range & N & Mean egg weight (g) & N \\
\hline Hatchery & 3,963 & 148 & $2,866-5,891$ & 25 & 0.23 & 25 \\
Natural & 3,946 & 295 & $2,842-4,951$ & 6 & 0.22 & 6 \\
\hline
\end{tabular}

\subsubsection{Spawning ground surveys}

Two spawning ground surveys were conducted from the WWNF boundary below Vey Meadows to Time and a Half Campground and from Time and a Half Campground to Spoolcart Campground on 5 July 2007. No redds or carcasses were observed. Standard spawning ground surveys in 2007 were conducted on 27 August, 4 September and 10 September. The only carcass recovered was a $690 \mathrm{~mm}$ FL female, 50\% spawned out, and unclipped was recovered in the Time and a Half Campground to Spoolcart Campground section on 4 September. Only one redd was observed, in the same section and on the same date as the carcass recovery. All but 3 of the 19 adults released above the trap to spawn naturally were natural-origin (Table 38). 
Table 38. Natural- and hatchery-origin spring Chinook salmon released above the upper Grande Ronde River trap in 2007 by FL group, sex, and origin.

\begin{tabular}{|c|c|c|c|c|}
\hline \multicolumn{2}{|c|}{ Hatchery } & \multirow[b]{2}{*}{ FL (cm) Group } & \multicolumn{2}{|c|}{ Natural } \\
\hline $\mathrm{M}$ & $\mathrm{F}$ & & $\mathrm{M}$ & $\mathrm{F}$ \\
\hline \multirow{11}{*}{3} & & $40-44$ & & \\
\hline & & $45-49$ & & \\
\hline & & $50-54$ & & \\
\hline & & $55-59$ & & \\
\hline & & $60-64$ & & \\
\hline & & $65-69$ & 3 & \\
\hline & & $70-74$ & 1 & 2 \\
\hline & & $75-79$ & 1 & 2 \\
\hline & & $80-84$ & & 3 \\
\hline & & 85-89 & 2 & 1 \\
\hline & & $90-94$ & 1 & \\
\hline 3 & 0 & Totals & 8 & 8 \\
\hline
\end{tabular}

\subsubsection{Run Reconstruction}

Only ages 4 and 5 returns comprised the age-length key for natural-origin returns collected in the trap (Table 39). Hatchery-origin were comprised of ages 3-5 (Table 40). Total returns to the stream were primarily ages 3 and 4 for hatchery-origin, and ages 4 and 5 for natural-origin (Table 41). Four of the five CWT groups represented in carcass recoveries were released from upper Grande Ronde acclimation facilities (Table 42). All of the upper Grande Ronde River release groups in Table 42 were conventional broodstock progeny.

High water temperatures probably resulted in mortality of most returns. Only one redd was observed above the trap, and none below. The 15 hatchery-origin prespawn mortalities recovered near the trap were 40-50 $(n=13), 67(n=1)$, and $79(n=1)$ cm FL. Three natural-origin mortalities recovered were $67(n=1), 70(n=1)$ and $75(n=1)$ cm FL. Hatchery-origin brood year 2002 smolt-to-adult ratio was 166/143,047, or 0.11\%. Natural-origin brood year 2002 smolt-to-adult ratio (returns divided by smolt equivalents) was $67 / 12,018$ or $0.56 \%$ 
Table 39. Age-length table for natural-origin spring Chinook salmon collected at the upper Grande Ronde River trap, 2007.

\begin{tabular}{ccccc}
\hline FL $(\mathrm{cm})$ & Age 3 & Age 4 & Age 5 & Totals \\
\hline 67 & 1 & & 1 \\
68 & 1 & & 1 \\
69 & 1 & & 1 \\
70 & 1 & & 1 \\
72 & 1 & & 1 \\
73 & 1 & & 1 \\
75 & 1 & & 1 \\
76 & 1 & & 1 \\
78 & & 1 & 1 \\
80 & & & 1 & 1 \\
81 & & & 2 & 2 \\
85 & & & 1 & 1 \\
88 & & & 1 & 1 \\
89 & & & 1 & 1 \\
94 & & & 1 & 1 \\
Totals & 0 & 8 & 8 & 16 \\
\hline
\end{tabular}


Table 40. Age-length table for hatchery-origin spring Chinook salmon collected at the upper Grande Ronde River trap, 2007.

\begin{tabular}{|c|c|c|c|c|}
\hline $\mathrm{FL}(\mathrm{cm})$ & Age 3 & Age 4 & Age 5 & Totals \\
\hline 40 & 1 & & & 1 \\
\hline 41 & 2 & & & 2 \\
\hline 44 & 1 & & & 1 \\
\hline 45 & 2 & & & 2 \\
\hline 46 & 1 & & & 1 \\
\hline 48 & 1 & & & 1 \\
\hline 49 & 1 & & & 1 \\
\hline 50 & 2 & & & 2 \\
\hline 59 & 1 & & & 1 \\
\hline 66 & & 1 & & 1 \\
\hline 68 & & 1 & & 1 \\
\hline 70 & & 1 & & 1 \\
\hline 71 & & 3 & & 3 \\
\hline 72 & & 4 & & 4 \\
\hline 73 & & 2 & & 2 \\
\hline 74 & & 5 & & 5 \\
\hline 75 & & 3 & & 3 \\
\hline 76 & & 1 & & 1 \\
\hline 77 & & 2 & & 2 \\
\hline 78 & & 3 & & 3 \\
\hline 79 & & 1 & & 1 \\
\hline 80 & & & 1 & 1 \\
\hline 82 & & 1 & & 1 \\
\hline 83 & & 1 & 1 & 2 \\
\hline 85 & & & 2 & 2 \\
\hline 87 & & & 1 & 1 \\
\hline 92 & & & 1 & 1 \\
\hline 93 & & & 2 & 2 \\
\hline Totals & 12 & 29 & 8 & 49 \\
\hline
\end{tabular}


Table 41. Catch at the upper Grande Ronde River trap (UGRT), disposition, and estimated total returns for adult spring Chinook salmon to the upper Grande Ronde River*, 2007.

\begin{tabular}{|c|c|c|c|c|c|c|}
\hline Stock/Rear ${ }^{\mathrm{a}}$ & Age $^{b}$ & UGRT & Mortality $^{\mathrm{c}}$ & Spawn Ab-UGRT $^{\mathrm{d}}$ & Spawn LH ${ }^{\mathrm{e}}$ & Total Returns \\
\hline Hatchery & 3 & 5 & 13 & 3 & 2 & 18 \\
\hline Natural & 3 & & & & & \\
\hline Totals & & 5 & 13 & 3 & 2 & 18 \\
\hline Hatchery & 4 & 30 & 3 & & 29 & 32 \\
\hline Natural & 4 & 18 & 3 & 8 & 10 & 21 \\
\hline Totals & & 48 & 6 & 8 & 39 & 53 \\
\hline Hatchery & 5 & 5 & & & 5 & 5 \\
\hline Natural & 5 & 15 & & 8 & 7 & 15 \\
\hline Totals & & 20 & 0 & 8 & 12 & 20 \\
\hline
\end{tabular}

* Rounded to nearest whole number

${ }^{a}$ UGR=upper Grande Ronde River hatchery-origin, Natural=Unmarked/untagged

${ }^{b}$ Age 3 UGR $<61 \mathrm{~cm} \mathrm{FL,} \mathrm{age} 4$ UGR $\geq 61 \leq 82 \mathrm{~cm} \mathrm{FL}$, age 5 UGR >82 cm FL, age 3 Natural <61 cm FL, age 4 Natural $\geq 61 \leq 77 \mathrm{~cm} \mathrm{FL,} \mathrm{age} 5 \mathrm{Natural}>77 \mathrm{~cm} \mathrm{FL}$

${ }^{c}$ Mortality at trap (1) or observed on prespawn surveys below the trap (18)

${ }^{d}$ Spawn Ab-UGRT=estimated spawners above the UGRT. No redds recorded below UGRT

'Spawn LH=conventional broodstock spawned at Lookingglass Hatchery or mortality while being held for spawning 
Table 42. Estimated hatchery-origin adult spring Chinook salmon returns to the upper Grande Ronde River and instream spawners* for 2007, by age, mark(s), and CWT group.

\begin{tabular}{cccccc}
\hline Age & Mark & CWT & Stock & Returns to Stream & Instream spawners \\
\hline 3 & None & 94213 & UGR & 18 & 3 \\
& & & & & 0 \\
4 & Ad-clip & 93824 & LKG & 1 & 0 \\
4 & VIE & 94035 & UGR & 12 & 0 \\
4 & VIE & 94036 & UGR & 19 & 0 \\
5 & & & & 5 & \\
\hline
\end{tabular}

* Rounded to nearest whole number

\subsubsection{Mean FL at Age}

Comparisons of mean FL at age were possible for 4 groups (Table 43). There was no significant differences in mean FL between hatchery- and natural-origin females at age 4 $(\mathrm{t}$ ratio $=-0.13523 \mathrm{P}>|\mathrm{t}|=0.8941)$, males at age $5(\mathrm{t}$ ratio $=-0.14646, \mathrm{P}>|\mathrm{t}|=0.8893)$, or females at age 5 ( $\mathrm{t}$ ratio $=-1.08092, \mathrm{P}>|\mathrm{t}|=0.3156)$. Mean FL at age 4 was significantly different between hatchery- and natural-origin males ( $\mathrm{t}$ ratio $=-2.37673, \mathrm{P}>$ $|t|=0.0295)$.

Table 43. FL summary by sex and origin for natural- and hatchery-origin upper Grande Ronde River spring Chinook salmon caught at the trap in 2007.

\begin{tabular}{ccccccc}
\hline Origin & Age & Sex & Mean FL $(\mathrm{mm})$ & SE & Range & N \\
\hline Hatchery & 3 & M & 469.7 & 15.3 & $406-594$ & 12 \\
Natural & 4 & M & 708.2 & 18.0 & $670-765$ & 5 \\
Hatchery & 4 & M & 760.2 & 11.4 & $682-835$ & 14 \\
Natural & 4 & F & 730.7 & 13.3 & $709-755$ & 3 \\
Hatchery & 4 & F & 733.0 & 7.2 & $666-783$ & 15 \\
Natural & 5 & M & 899.0 & 27.7 & $852-948$ & 3 \\
Hatchery & 5 & M & 904.5 & 25.0 & $830-935$ & 4 \\
Natural & 5 & F & 819.4 & 16.8 & $785-883$ & 5 \\
Hatchery & 5 & F & 843.8 & 13.8 & $805-870$ & 4 \\
\hline
\end{tabular}

\subsubsection{Environmental Data}

Maximum daily water temperatures during the release period at the upper Grande Ronde River acclimation site (19 March-11 April 2007) ranged from $5.4-10.5^{\circ} \mathrm{C}$ (Figure 28). 


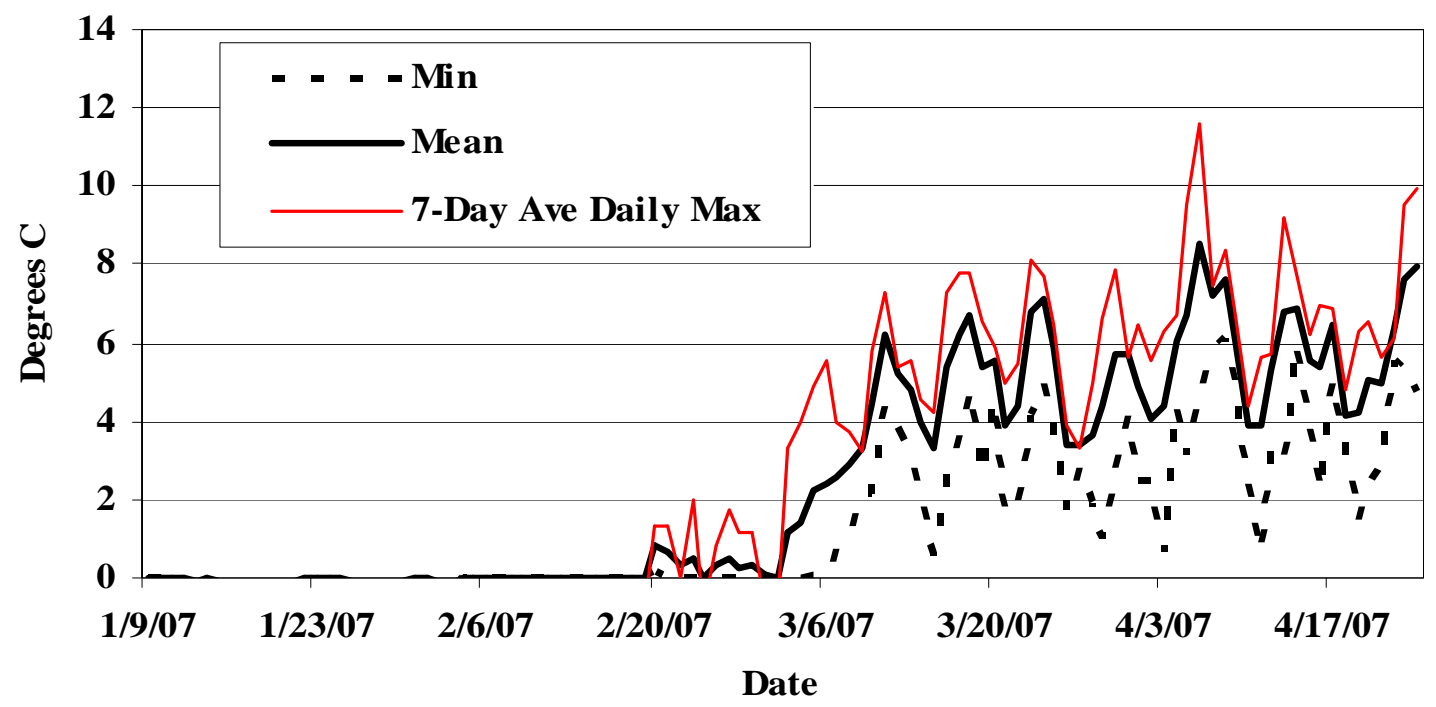

Figure 28. Daily minimum, mean, and maximum water temperatures at the upper Grande Ronde River acclimation site, 2007.

The highest daily water temperature recorded at the upper Grande Ronde River below Clear Creek site in 2007 was $29.7^{\circ} \mathrm{C}$ on 23 July (Figure 29). The maximum daily fluctuation was $22.5^{\circ} \mathrm{C}$ on 1 August. 


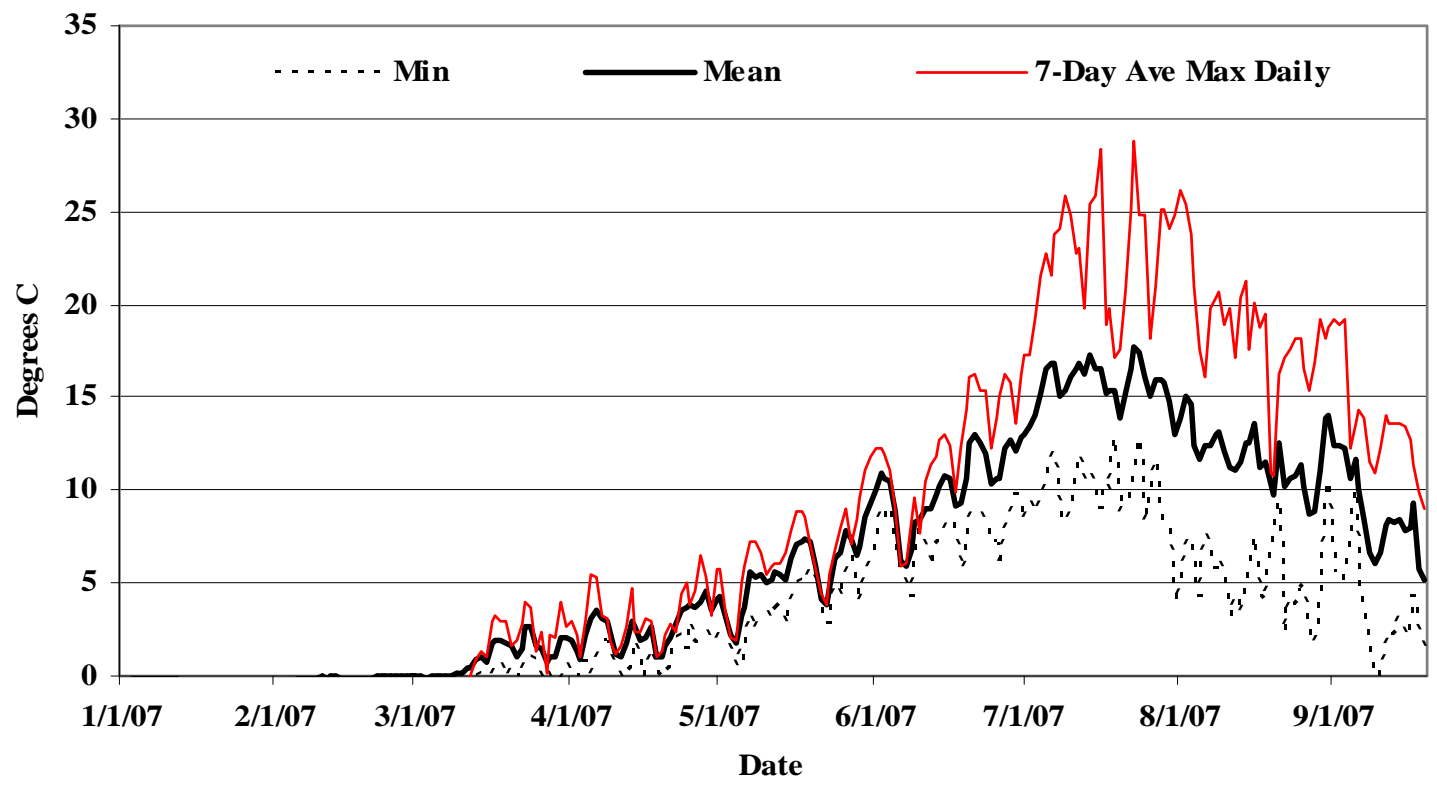

Figure 29. Daily minimum, mean, and 7-day average maximum water temperatures at the upper Grande Ronde River below Clear Creek site, 2007 (data courtesy WWNF).

The highest daily water temperature recorded at the upper Grande Ronde River adult site in 2007 was $30.1^{\circ} \mathrm{C}$ on 23 July (Figure 30). The maximum daily fluctuation was $16.2^{\circ} \mathrm{C}$ on 31 July and 1 August. 


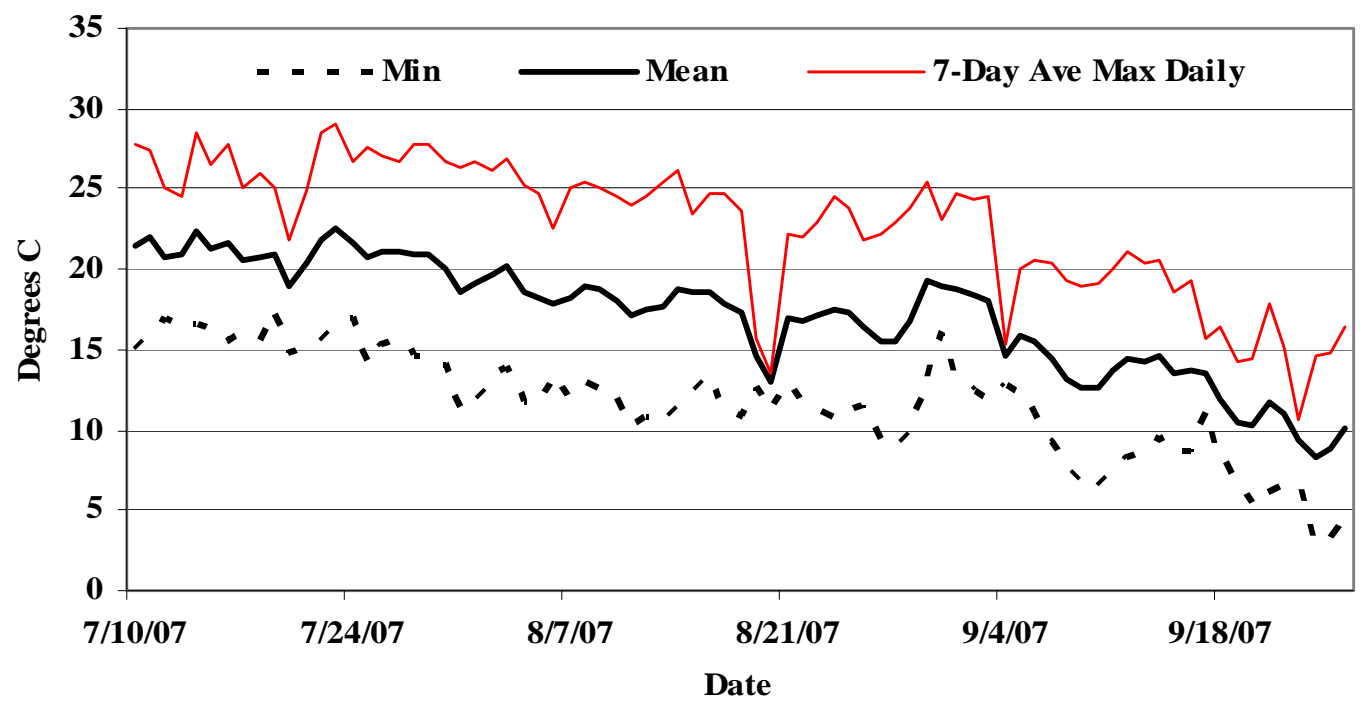

Figure 30. Daily minimum, mean, and 7-day average maximum daily maximum water temperatures at the upper Grande Ronde adult site, 2007.

\subsection{Discussion}

Differences in life history were evident between hatchery- and natural-origin outmigrants in 2007 and similar to past years. Hatchery-origin juveniles are reared to a larger size than natural-origin and released during a narrow window of time in the spring to maximize survival. Release times and size at release are also affected by other factors such as availability of rearing space for different life stages. Managers may also alter the release time in the spring to avoid having a large group of hatchery-fish causing naturalorigin fish to outmigrate earlier than they would normally ("Pied Piper effect"). These tradeoffs produce many of the differences in life history observed. The differences in life history of hatchery-origin juveniles may also have effects on adults returning, such as increased returns of age 3 males. There were few differences between life histories of hatchery- and natural-origin adults, most noticeably age structure and length-at-age of returns.

Survival of spring Chinook salmon outmigrants in the hydrosystem in the spring of 2007 was relatively high, given the lower than normal flows. This was attributed to high levels of 24 hour spill in low flow conditions and the delayed start of the transport program resulting in a smaller proportion of the run being transported (DeHart 2007). 
The numbers of returning adults has responded positively, with most being hatcheryorigin. However, SAR of hatchery releases remains below goals. Past SAR values of hatchery-origin fish for both Catherine Creek and the upper Grande Ronde River were below the LSRCP/NEOH goal of $0.65 \%$ (Hesse et al. 2006) and the range of 2-6\% for Snake River salmon and steelhead proposed by the Northwest Power and Conservation Council (NPCC 2003). The estimated BY 2002 SAR for both Catherine Creek and the upper Grande Ronde River hatchery releases were also well below these goals.

Substantial prespawning mortality (primarily jacks, but also some age 4/5) occurred in the upper Grande Ronde River in 2007 (McLean et al. 2007), probably due to stress from elevated water temperatures, or multiple factors. The number recovered during a small section of stream near the trap was over $10 \%$ of the total number trapped, so the total number succumbing prior to spawning in the stream was likely more. Poor water quality (excessive water temperatures just prior to and during spawning) has been a frequent problem in the upper Grande Ronde River.

Progeny-per parent for Catherine Creek for brood year 2002 was well below replacement (1.0). There was no value estimated for the upper Grande Ronde River due to the low numbers of hatchery-origin smolts released in 2000 and 2001 and the low numbers of age 4 hatchery-origin returns in 2002.

The expanded numbers of returns to both Catherine Creek and the upper Grande Ronde River as shown in RMIS are in error, and need to be expanded properly to account for the total number of returns to the streams.

\subsection{Literature Cited}

Analytical Software. 2000. Statistix 7 for Windows. Tallahassee, Florida.

Bauman, L., J. Mead, and C. Stein (eds). 1998. PIT Tag Information System User Manual. Version 4. Pacific States Marine Fisheries Commission. Portland, Oregon.

Bouwes, N., C. Petrosky, H. Schaller, P. Wilson, E. Weber, S. Scott, and R. Boyce. 2002. Comparative Survival Rate Study (CSS) of Hatchery PIT-tagged Chinook Status Report for Migration Years 1997-2000 Mark/Recapture Activities, 2001 Annual Report to the Bonneville Power Administration, Project 199602000, Portland, Oregon.

DeHart, M. 2007. Annual Report of the Fish Passage Center. Columbia Basin Fish and Wildlife Authority. Portland, Oregon.

Flagg, T. A., B. A. Berejikian, J. E. Colt, W. W. Dickhoff, L. W. Harrell, D. J. Maynard, C. E. Nash, M. S. Strom, R. N. Iwamoto, and C. V. W. Mahnken. 2000. Ecological and 
behavioral impacts of artificial production strategies on the abundance of wild salmon populations. U. S. Department of Commerce, National Oceanic and Atmospheric Administration, National Marine Fisheries Service, Northwest Fisheries Science Center, NOAA Technical Memorandum NMFS/NWFSC-41, Seattle, Washington.

Hesse, J. A., J. R. Harbeck, and R. W. Carmichael. 2006. Monitoring and evaluation plan for Northeast Oregon hatchery Imnaha and Grande Ronde subbasin spring Chinook salmon. Nez Perce Tribe, Lapwai, Idaho, and Oregon Department of Fish and Wildlife, La Grande, Oregon.

Jonasson, B. C., J. V. Tranquilli, P. Sankovich, E. S. Van Dyke, M. Keefe, and R. W. Carmichael. 2002. Investigations into the early life history of naturally produced spring Chinook salmon in the Grande Ronde River basin. Project Period: 1 September 1998-31 August 2002. Annual Report to the Bonneville Power Administration, Project 199202604, Portland, Oregon.

McLean, M. L., R. Seeger, and L. Hewitt. 2006. Grande Ronde Satellite Facilities O\&M: 1 January 2006-31 December 2006. Annual Report to the Bonneville Power Administration, Project 199800703, Contract 25348.

Mosher, K. H. 1969. Identification of Pacific Salmon and Steelhead Trout by Scale Characteristics. U. S. Department of the Interior, Fish and Wildlife Service, Bureau of Commercial Fisheries Circular 317.

Nehlsen, W., J. E. Williams, and J. A. Lichatowich. 1991. Pacific salmon at the crossroads: stocks at risk from California, Oregon, Idaho, and Washington. Fisheries 16:4-21.

NPCC (Northwest Power and Conservation Council). 2003. Mainstem Amendments to the Columbia River Basin Fish and Wildlife Program. Council Document 2003-11. Portland, Oregon.

Park, D. L. 1990. Status and future of spring Chinook salmon in the Columbia River Basin - Conservation and Enhancement. U. S. Department of Commerce, NOAA Technical Memorandum NMFS F/NWC-187.

Parker, S. J., M. Keefe, and R. W. Carmichael. 1995. Natural escapement of spring Chinook salmon in the Imnaha and Grande Ronde River Basins. Annual Progress Report to the Lower Snake River Compensation Plan, U. S. Fish and Wildlife Service, Boise, Idaho. 
Ricker, W. E. 1975. Computation and interpretation of biological statistics of fish populations. Bulletin 191 of the Fisheries Research Board of Canada. Ottawa.

SAS Institute, Inc. 2006. JMP Statistics and Graphics Guide, Release 6. SAS Institute, Cary, North Carolina.

Skalski, J. R. and R. L. Townsend. 2006. Pacific Northwest Hatcheries Smolt-to-Adult Ratio (SAR) Estimation using Coded Wire Tags (CWT) Data. Report to the Bonneville Power Administration, Project 199105100, Portland, Oregon.

Westhagen, P. and J. R. Skalski. 2006. Program PitPro 4.0. Columbia Basin Research, University of Washington, Seattle.

Zimmerman, B. and S. Patterson. 2002. Grande Ronde Basin Spring Chinook Hatchery Management Plan. Confederated Tribes of the Umatilla Indian Reservation, Pendleton, Oregon.

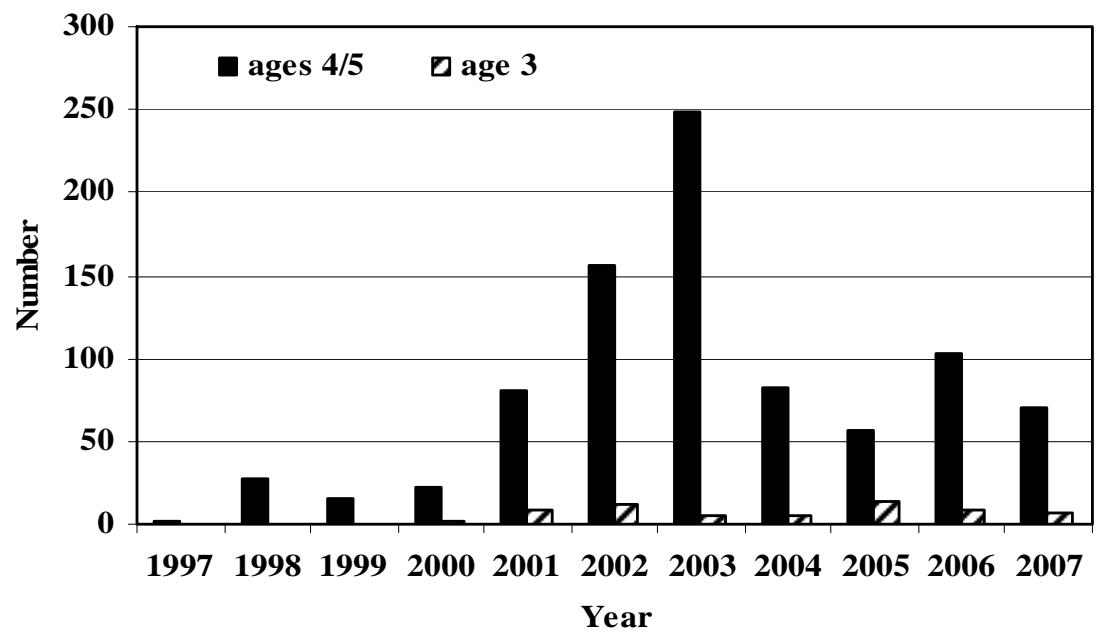

Appendix Figure A1. Natural-origin spring Chinook salmon collected at the Catherine Creek trap, 1997-2007. 


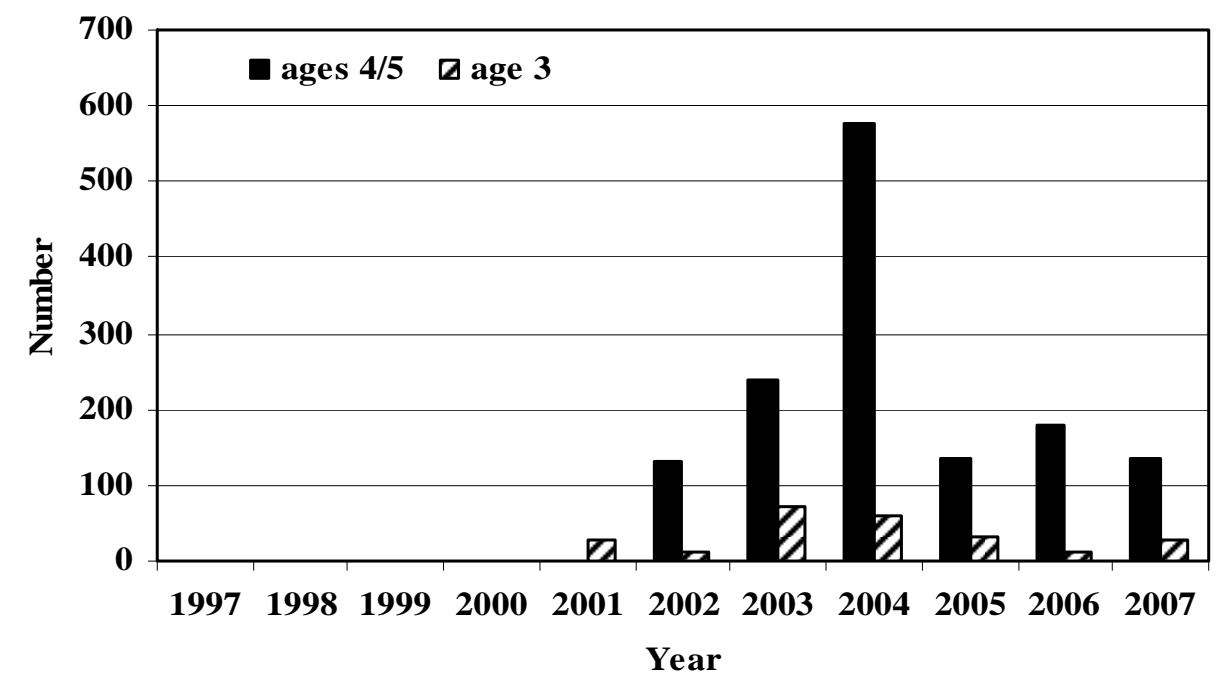

Appendix Figure A2. Hatchery-origin spring Chinook salmon collected at the Catherine Creek trap, 1997-2007.

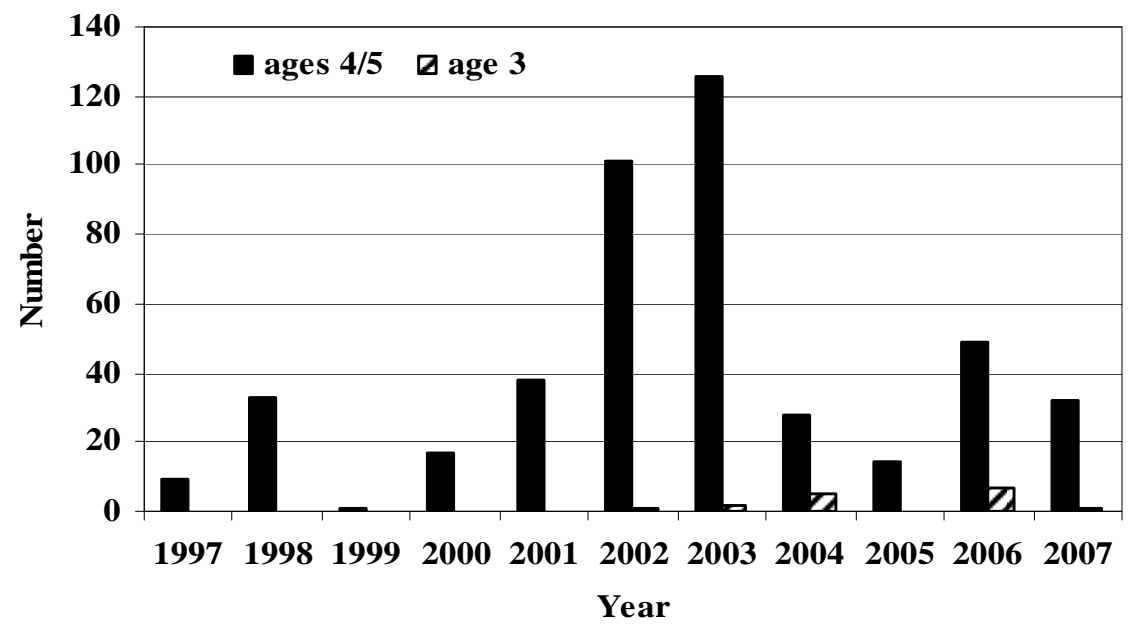

Appendix Figure A3. Natural-origin spring Chinook salmon collected at the upper Grande Ronde River trap, 1997-2007. 


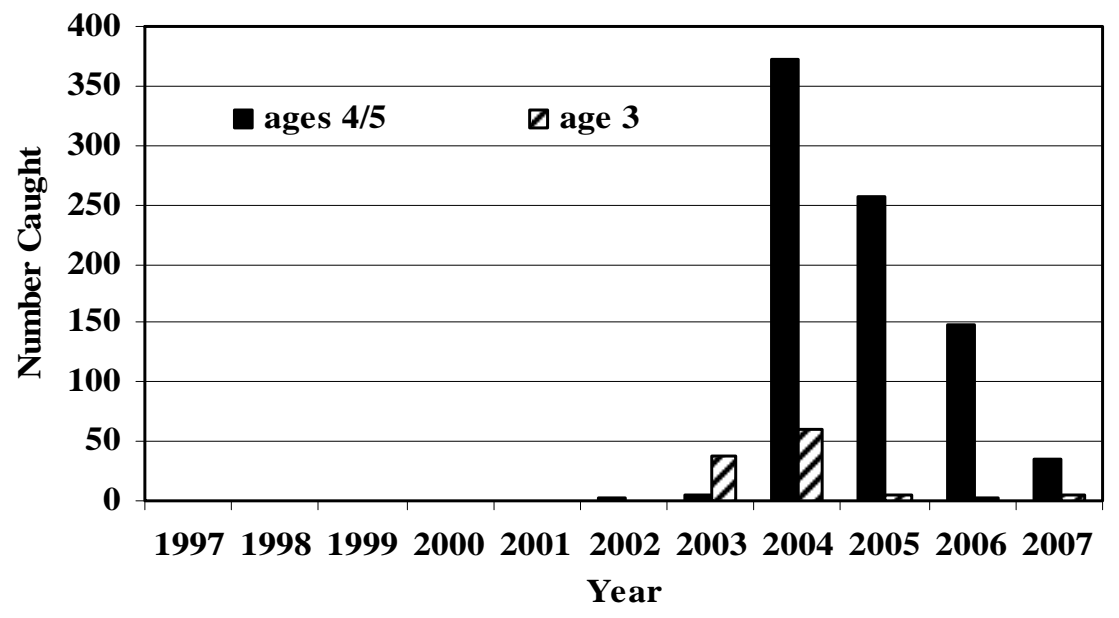

Appendix Figure A4. Hatchery-origin spring Chinook salmon collected at the upper Grande Ronde River trap, 1997-2007.

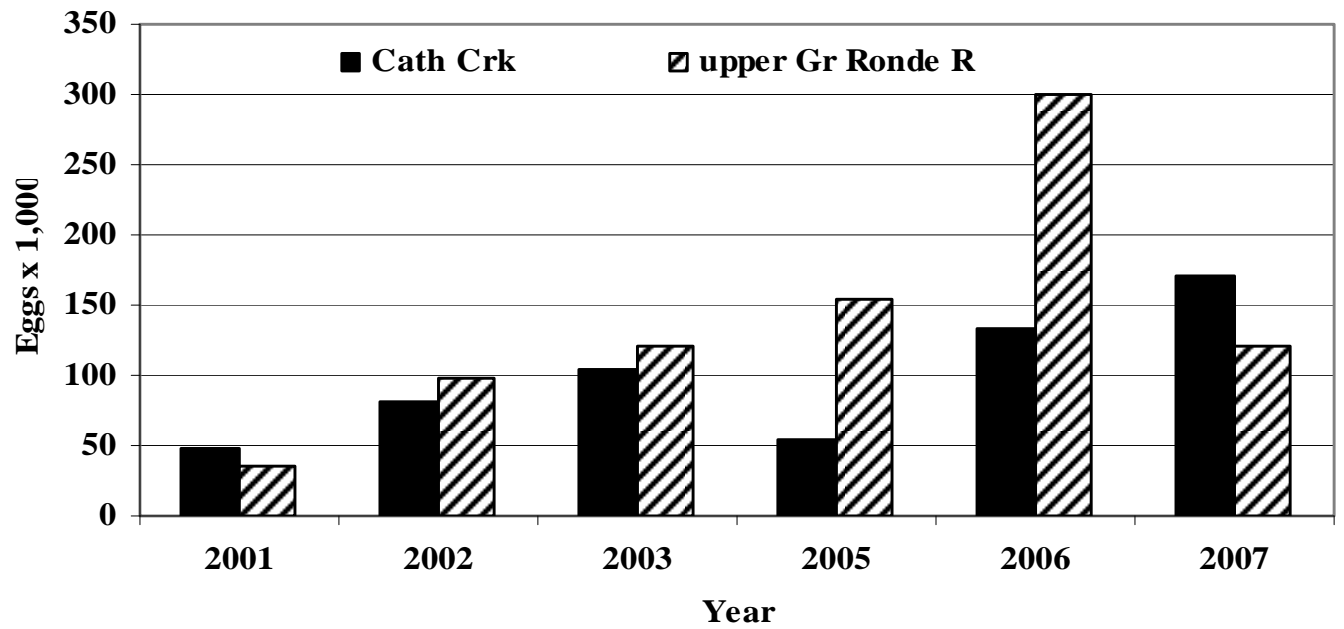

Appendix Figure A5. Egg production from conventional broodstock spring Chinook salmon collected from the Catherine Creek and upper Grande Ronde River traps, 20012007. 


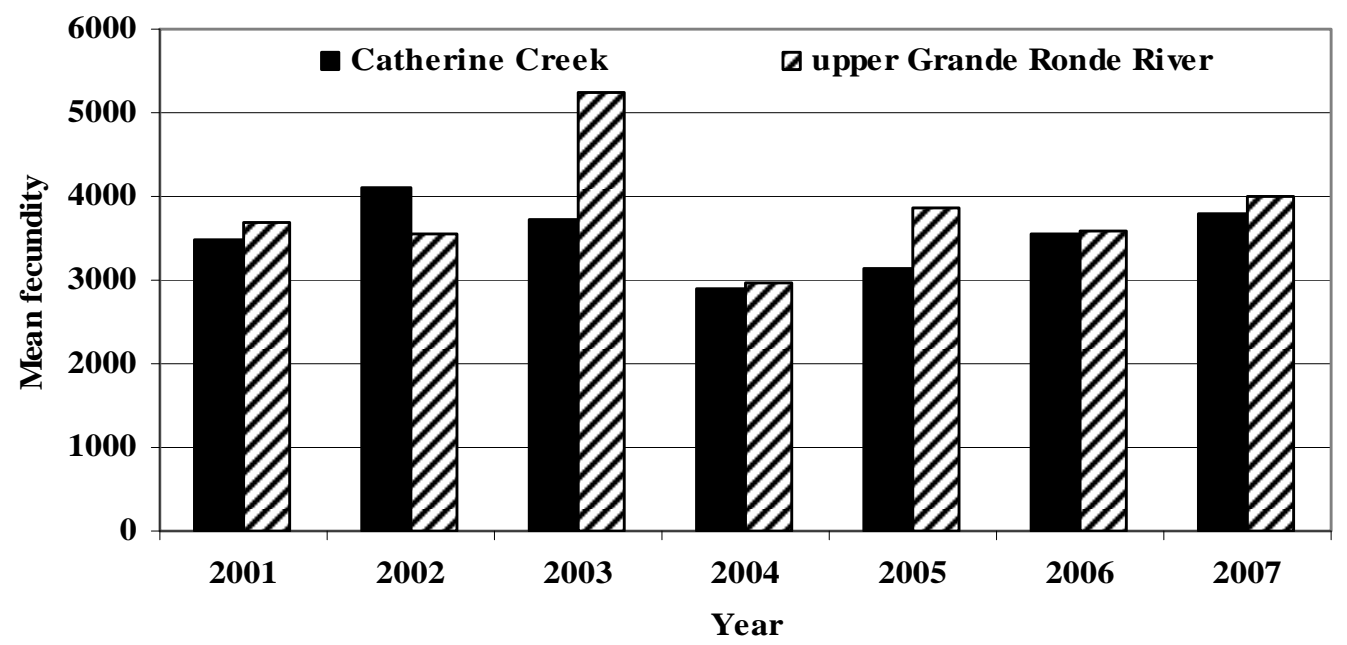

Appendix Figure A6. Mean fecundity (eggs/female) for conventional broodstock spring Chinook salmon collected from the Catherine Creek and upper Grande Ronde River traps, 2001-2007.

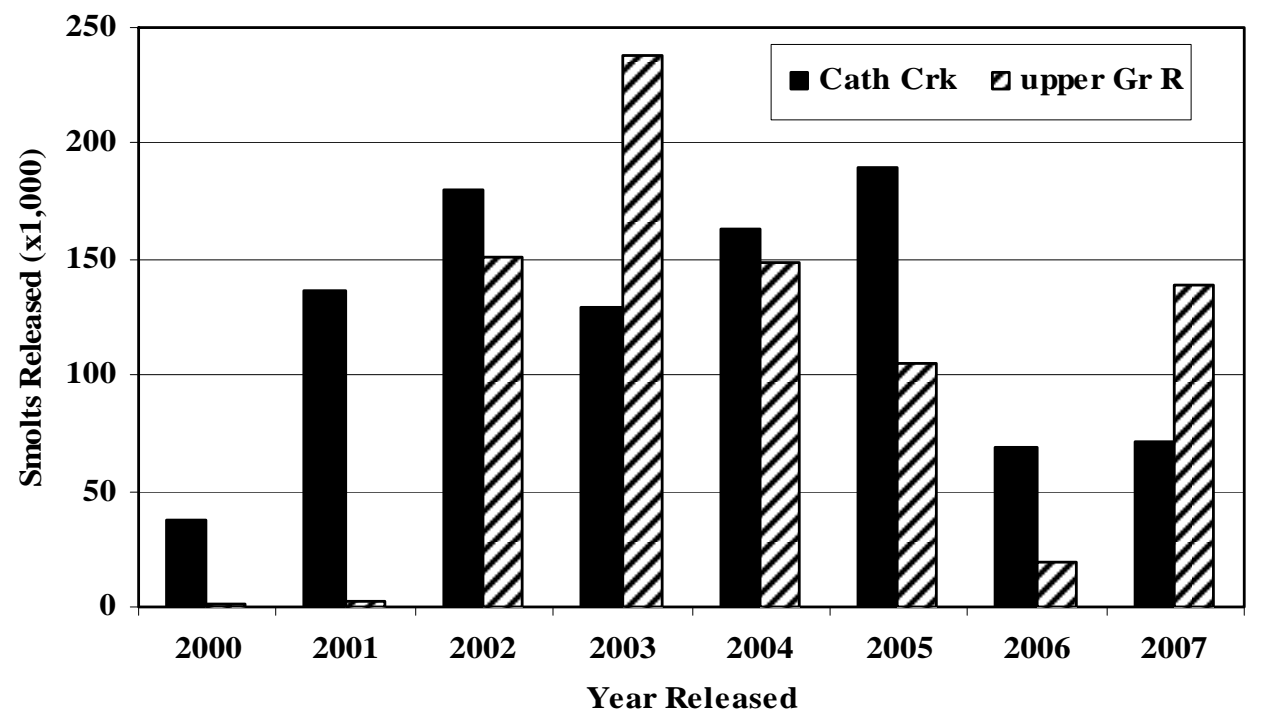

Appendix Figure A7. Hatchery-origin spring Chinook salmon smolt releases from Catherine Creek and upper Grande Ronde River acclimation facilities (captive and conventional broodstocks), 2000-2007. 


\section{SECTION II. SUMMER STEELHEAD IN CATHERINE CREEK AND THE UPPER GRANDE RONDE RIVER, OREGON}

\section{$2.1 \quad$ Executive Summary}

This is the ninth annual report for a multi-year project designed to monitor and evaluate supplementation of endemic spring Chinook salmon and describe adult life history of summer steelhead in Catherine Creek and the upper Grande Ronde River. These two streams have historically supported anadromous fish populations that provided significant tribal and non-tribal fisheries. The presence of effective adult salmonid collection facilities provides an opportunity to collect basic life history data for adult summer steelhead and give managers the background needed for recovery planning. The 2007 Statement of Work included describing life history characteristics of adult summer steelhead from Catherine Creek and the upper Grande Ronde River.

This project included operations and maintenance (O\&M) and monitoring and evaluation (M\&E) components prior to 2002. Beginning in 2002, project O\&M responsibilities were removed and placed in a separate project (Bonneville Power Administration (BPA) Project 199800703, BPA Contract No. 0006509, CTUIR Project 410), with a separate budget and reporting requirements. The two sections of this annual report include spring Chinook salmon monitoring and evaluation and summer steelhead life history (BPA Project 199800703, BPA Contract No. 00012785, CTUIR Project 413).

Upstream-migrating, prespawn wild summer steelhead captured at the Catherine Creek trap from 12 March-9 May 2007 totaled 128 (48 males, 80 females). FL ranged from 521-783 mm and age composition was 71 1-ocean and 57 2-ocean. Fallbacks collected from 26 March-16 July totaled 22 (10 males, 12 females); all had been previously collected at the trap and marked with opercle punches. Estimated escapement above the trap was 129 (95\% confidence interval 128-147. No ad-clipped (hatchery-origin) or identifiable strays from other streams were caught.

Upstream-migrating, prespawn wild summer steelhead captured at the upper Grande Ronde River trap from 4 April-18 May totaled 65 (32 males, 33 females. FL ranged from 512-792 mm and age composition was 35 1-ocean and 30 2-ocean. Fallbacks collected from 11 May-3 June totaled 18 (6 males, 12 females); all had been previously collected at the trap and marked with opercle punches. Estimated escapement above the trap was 66 (95\% confidence interval 65-81). No ad-clipped (hatchery-origin) adults were collected. One fish tagged at Sherars Falls (Deschutes River) on 27 July 2006 was collected as both an upstream migrant and fallback. 
Life history characteristics of summer steelhead collected from Catherine Creek and the upper Grande Ronde River in 2007 were similar to those observed in previous years for these streams and similar to the limited data available for other populations of A-run summer steelhead in the Snake River Basin.

\section{$2.2 \quad$ Introduction}

Wild steelhead populations in the Snake River basin have declined along with other anadromous species. Sport fisheries in the Grande Ronde and Imnaha Rivers were closed in 1974 (Flesher et al. 2000). Snake River summer steelhead were listed as threatened under the Endangered Species Act in 1997. Managers have augmented summer steelhead populations with hatchery-reared juveniles since 1978 to restore sport fisheries in Catherine Creek, the upper Grande Ronde River, and other streams in the Grande Ronde Basin (Carmichael et al. 1990). Creel surveys since 1985 show this program has been very successful (Flesher et al. 2000). However, the stock used (Wallowa Hatchery), for reasons poorly understood, has a high rate of straying into the Deschutes River. The National Marine Fisheries Service directed use of Wallowa stock be phased out by 2008 (NMFS 1999). A lack of basic life history data for Grande Ronde Basin summer steelhead has limited the development of management alternatives to aid in population recovery. The existence of adult collection weirs on Catherine Creek, the upper Grande Ronde River, and other streams in the basin provide opportunities to collect valuable adult life history information during the spawning migration that occurs during FebruaryJuly.

\subsection{Methods}

Adult summer steelhead life history metrics included migration timing (arrival at traps), age, abundance, migration status (upstream (prespawn) or fallbacks (postspawn)), length and sex frequencies, occurrence of strays and hatchery/wild ratios. Population estimates were made for spawners above the trap by marking all returns collected at the traps with an opercle punch before passing upstream and observing fallbacks on the weir as recaptures. We used the Chapman modification of the Petersen method (Ricker 1975) with the upper (95\%) bounds using Appendix II of Ricker (1975) and the actual numbers caught at each trap for the lower confidence bounds. Tissues (from opercle punches) were collected, preserved in vials with 95\% ethanol, and sent to CRITFC (Columbia River Inter-Tribal Fisheries Commission) for storage. We used descriptive statistics in tabular and graphical form to summarize data. 


\section{$2.4 \quad$ Results}

\subsubsection{Catherine Creek}

The Catherine Creek adult trap began operation on 1 March 2007. The first wild, upstream-migrating (prespawn) female summer steelhead was collected on 14 March and the last on 9 May. The first male was collected on 12 March and the last on 30 April. There were no ad-clipped (hatchery-origin) adults collected, and none identifiable as strays from other streams by PIT or other tags.

The largest numbers of upstream-migrants were collected the weeks of 19 March, 9 April, and 23 April, with approximately 56\% caught during those three weeks (Table 1). Female to male ratio was 1.67:1. There were 22 fallbacks (postspawn) captured, most during the weeks of 7 and 14 May. Females were caught from 26 March-4 June and males from 7 May-16 July. Females were usually 54-71 cm (Figure 1). The FL frequency distribution for males was more dispersed among the FL cm categories.

Minimum daily water temperatures ranged from $0.4-6.3{ }^{\circ} \mathrm{C}$ during the period when upstream-migrating adults were caught (Figure 2). Slightly over half of the days (35 of 61) were in the range of 2.1 to $4.0^{\circ} \mathrm{C}$. The 7-day average daily maximums ranged from 3.2-12. ${ }^{\circ} \mathrm{C}$. Weeks of peak catches (26 March, 9 April, and 23 April) did not show any characteristics that distinguished them from any other days in the period. Stream flow data for the North Fork Catherine Creek site for 2007 were not available.

The size range of upstream-migrating females and mean FL were greater than for males (Table 2) and the same occurred for fallbacks (Table 3). Mean FL of 1-ocean and 2ocean males were greater than females (Table 4). The run was dominated by 1-ocean adults (Figure 3).

All 22 fallbacks were recovered dead on the weir and had opercle punches. The population estimate of summer steelhead passed above the weir was 129 (95\% confidence interval 128-147). The actual number caught at the weir was substituted for the lower confidence limit. 
Table 1. Summer steelhead captured at the Catherine Creek trap by week, migration status and sex, 2007.

\begin{tabular}{|c|c|c|c|c|c|c|}
\hline \multirow[b]{2}{*}{ Week } & \multicolumn{3}{|c|}{ Upstream-migrating } & \multicolumn{3}{|c|}{ Fallbacks } \\
\hline & Male & Female & Total & Male & Female & Total \\
\hline $3 / 12$ & 15 & 3 & 18 & & & \\
\hline $3 / 19$ & 11 & 15 & 26 & & & \\
\hline $3 / 26$ & 4 & 9 & 13 & & 1 & 1 \\
\hline $4 / 2$ & & 2 & 2 & & & \\
\hline $4 / 9$ & 7 & 18 & 25 & & & \\
\hline $4 / 16$ & 7 & 4 & 11 & & 2 & 2 \\
\hline $4 / 23$ & 3 & 18 & 21 & & & \\
\hline $4 / 30$ & 1 & 9 & 10 & & & \\
\hline $5 / 7$ & & 2 & 2 & 4 & 2 & 6 \\
\hline $5 / 14$ & & & & 4 & 5 & 9 \\
\hline $5 / 21$ & & & & 1 & & 1 \\
\hline $5 / 28$ & & & & & & \\
\hline $6 / 4$ & & & & & 2 & 2 \\
\hline $6 / 11$ & & & & & & \\
\hline $6 / 18$ & & & & & & \\
\hline $6 / 25$ & & & & & & \\
\hline $7 / 2$ & & & & & & \\
\hline $7 / 9$ & & & & & & \\
\hline $7 / 16$ & & & & 1 & & 1 \\
\hline Totals & 48 & 80 & 128 & 10 & 12 & 22 \\
\hline
\end{tabular}




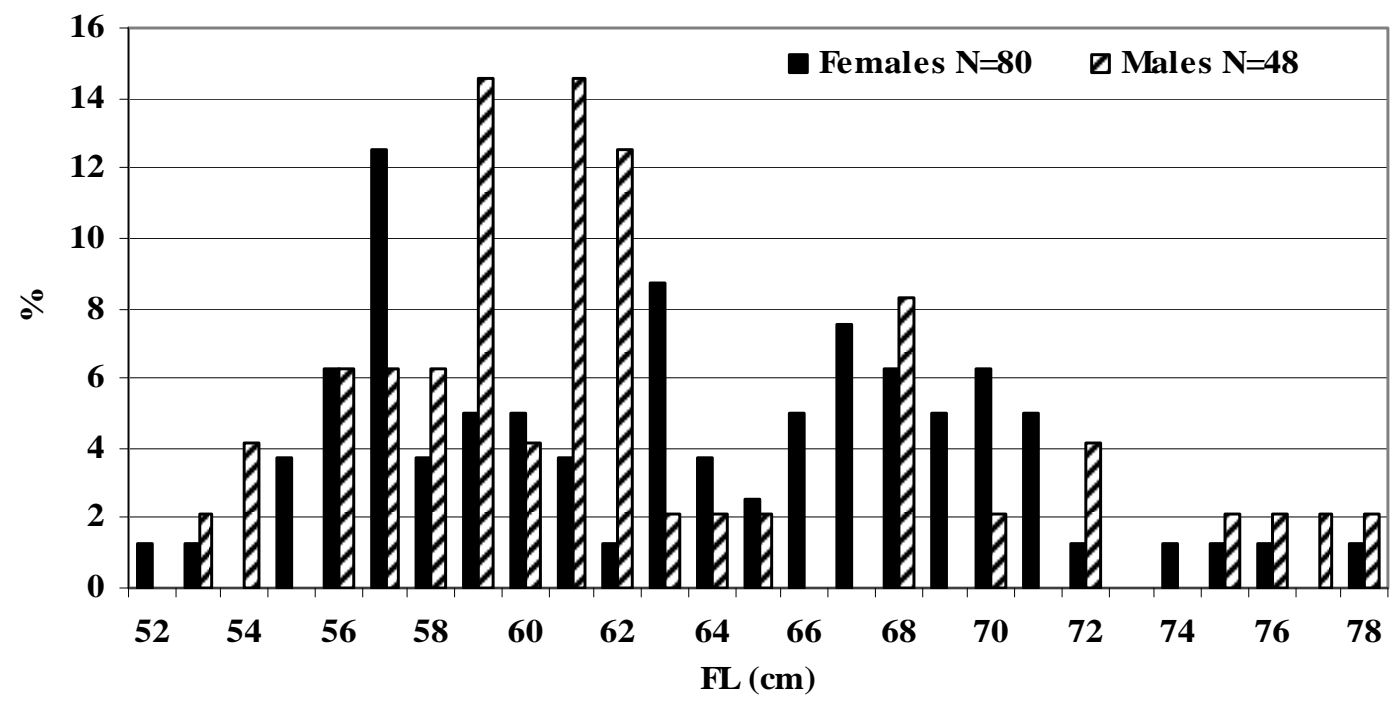

Figure 1. FL distribution by sex of summer steelhead caught at the Catherine Creek trap, 2007.

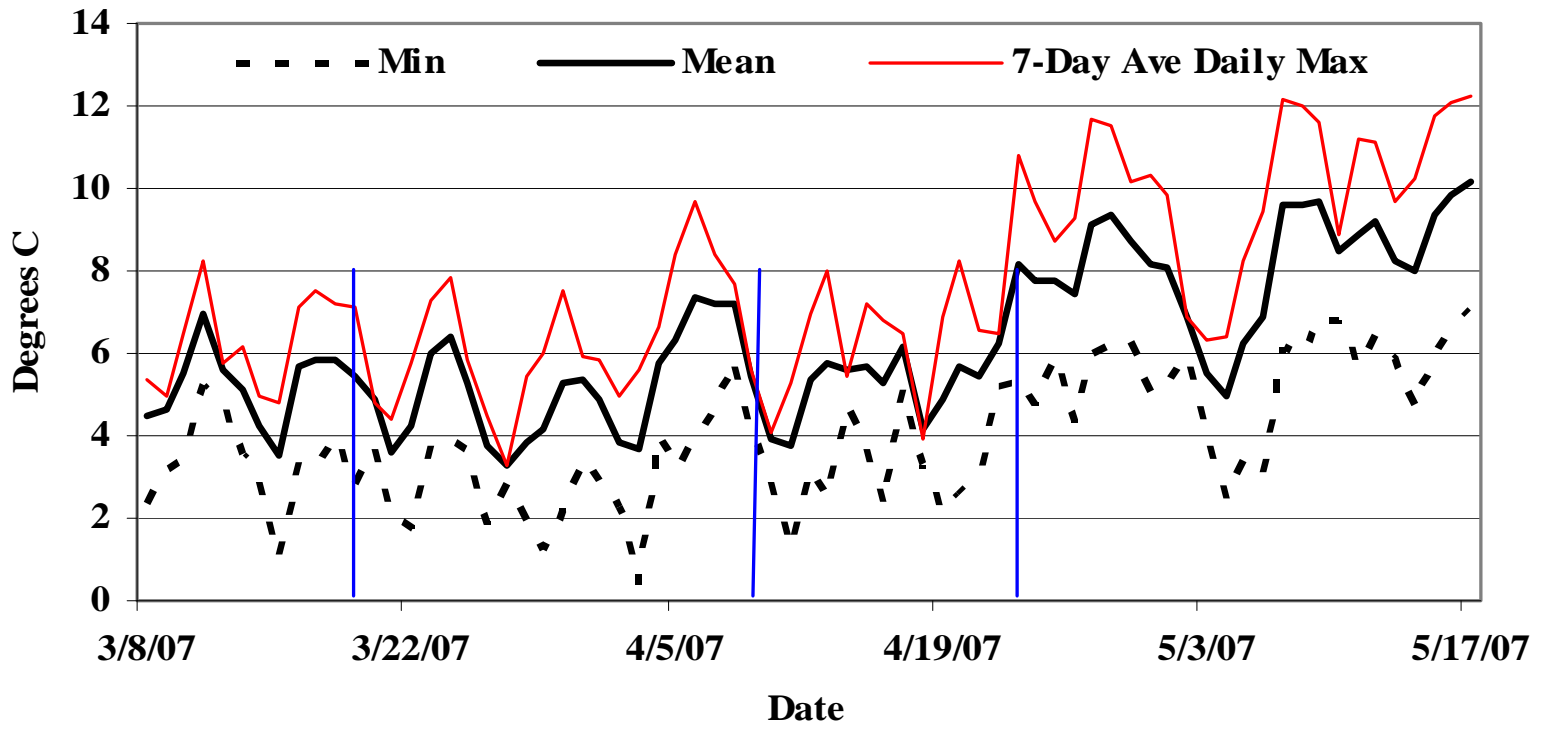

Figure 2. Water temperatures at the Catherine Creek adult trap encompassing the dates of first and last capture of upstream-migrating summer steelhead, 2007. 
Table 2. FL summary by sex for upstream-migrating summer steelhead collected at the Catherine Creek trap, 2007 (first-time captures only).

\begin{tabular}{lcccc}
\hline Sex & Mean FL $(\mathrm{mm})$ & SE & Range & $\mathrm{N}$ \\
\hline Male & 626.7 & 8.8 & $539-781$ & 48 \\
Female & 637.6 & 6.7 & $521-783$ & 80 \\
Total & 633.5 & 5.3 & $521-783$ & 128 \\
\hline
\end{tabular}

Table 3. FL summary by sex for fallback summer steelhead collected at the Catherine Creek trap, 2007.

\begin{tabular}{lcccc}
\hline Sex & Mean FL $(\mathrm{mm})$ & SE & Range & N \\
\hline Male & 634.0 & 15.2 & $567-714$ & 10 \\
Female & 666.5 & 13.2 & $590-730$ & 12 \\
Total & 651.7 & 10.4 & $567-730$ & 22 \\
\hline
\end{tabular}

Table 4. FL at age summary by age and sex for summer steelhead collected at the Catherine Creek trap, 2007.

\begin{tabular}{lcccccc}
\hline Age & Sex & Mean FL $(\mathrm{mm})$ & SE & Min & Max & N \\
\hline 1-ocean & M & 597.8 & 5.4 & 539 & 680 & 32 \\
& F & 581.0 & 4.4 & 521 & 633 & 30 \\
& Combined & 589.7 & 3.7 & 521 & 680 & 62 \\
2-ocean & M & 717.0 & 14.3 & 642 & 781 & 11 \\
& F & 682.3 & 6.4 & 583 & 783 & 41 \\
& Combined & 689.6 & 6.1 & 583 & 783 & 52 \\
\hline
\end{tabular}




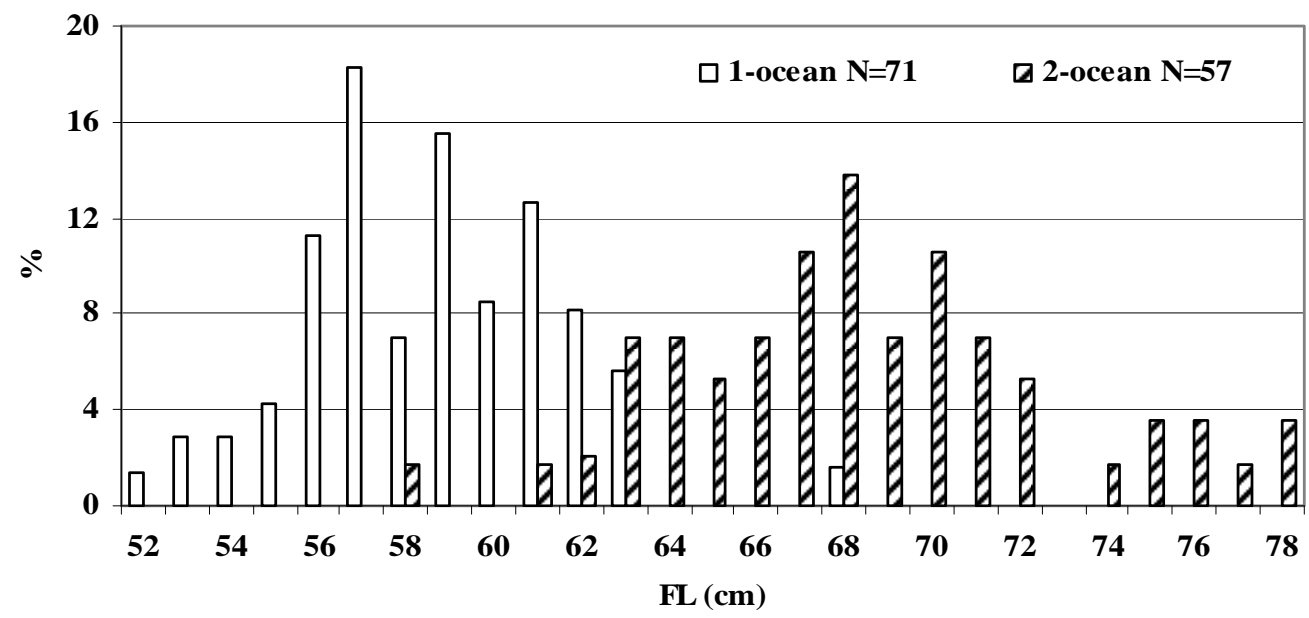

Figure 3. Age-FL (cm) key results for Catherine Creek summer steelhead, 2007.

\subsubsection{Upper Grande Ronde River}

The adult trap on the upper Grande Ronde River began operation on 15 March 2007. The first upstream-migrating female was caught on 4 April and the last on 18 May. The first male was caught on 6 April and the last on 11 May. A total of 65 were caught (33 females, 32 males). Approximately $75 \%$ of the upstream-grating adults were caught the weeks of 23 and 30 April (Table 5). Fallbacks caught from 11 May-3 June totaled 18. There were no ad-clipped (hatchery-origin) adults collected. One adult passed upstream and later recovered as a mortality on the weir had been externally tagged ( $\mathrm{T}$ anchor) on 27 July 2006 at Sherars Falls on the Deschutes River.

FL (cm) distributions of males and females were similar (Figure 4). Mean FL of males and females differed by $5.9 \mathrm{~mm}$ (Table 6). Mean FL of male fallbacks were 71.7 longer than females (Table 7). Mean FL of 1-ocean adults differed by $4 \mathrm{~mm}$ by sex; the difference increased to $12.4 \mathrm{~mm}$ for 2-ocean adults (Table 8). Upstream migrants were primarily 1-ocean and there was some overlap in the FL distributions of 1-ocean and 2ocean adults (Figure 5). All 18 fallbacks were recovered dead on the weir and had opercle punches. The population estimate of summer steelhead passed above the weir was 66 (95\% confidence interval 65-81). The actual number caught at the weir was substituted for the lower bound. Water temperature data for the upper Grande Ronde River adult trap site were not collected for the summer steelhead trapping season in 2007 and stream flow data for the site near Clear Creek were not available for 2007 when this report was written. 
Table 5. Summer steelhead captured at the upper Grande Ronde River trap by week, migration status, and sex, 2007.

\begin{tabular}{lcccccc}
\hline & \multicolumn{3}{c}{ Upstream-migrating } & \multicolumn{3}{c}{ Fallbacks } \\
\cline { 2 - 7 } Week & Male & Female & Total & Male & Female & Total \\
\hline $4 / 2$ & 1 & 2 & 3 & & & \\
$4 / 9$ & 2 & 1 & 3 & & & \\
$4 / 16$ & & & & & & \\
$4 / 23$ & 17 & 8 & 25 & & & \\
$4 / 30$ & 10 & 14 & 24 & & 1 & 1 \\
$5 / 7$ & 2 & 2 & 4 & 3 & 1 & 2 \\
$5 / 14$ & & 5 & 5 & 2 & 4 & 9 \\
$5 / 21$ & & & & 6 & 12 & 18 \\
$5 / 28$ & & 1 & 1 & & & \\
Totals & 32 & 33 & 65 & & & \\
\hline
\end{tabular}

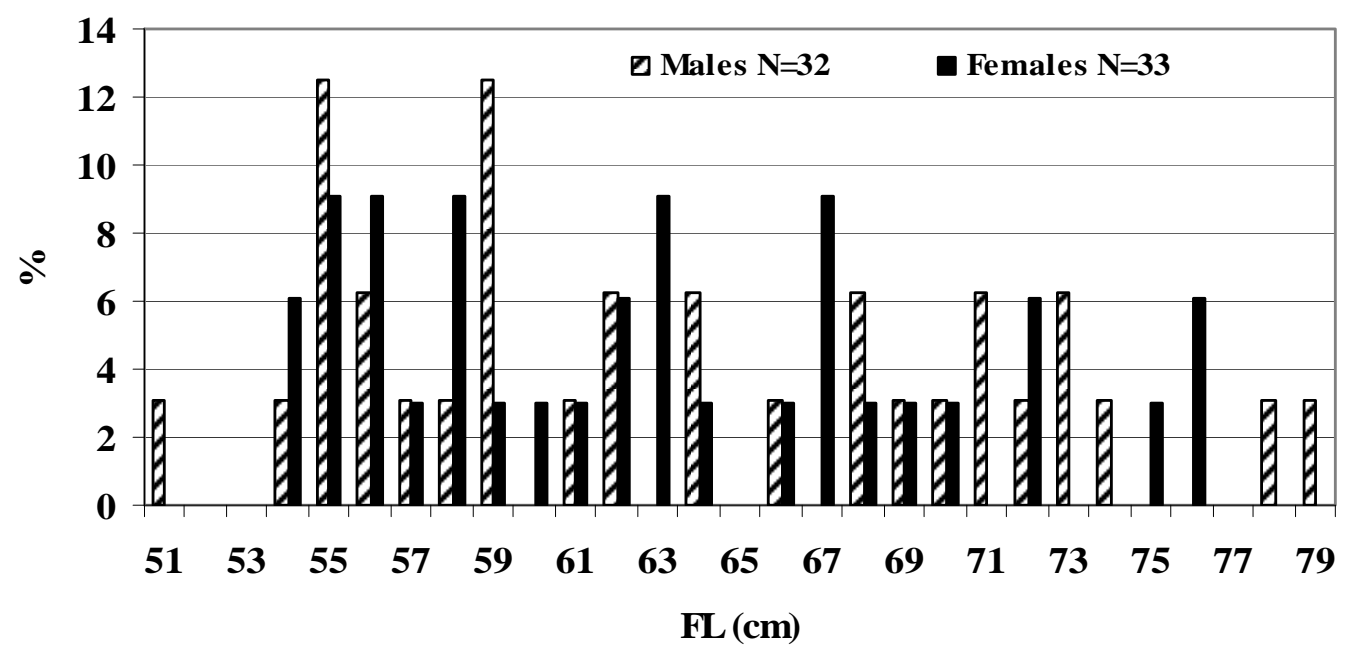

Figure 4. FL distribution by sex of summer steelhead caught at the upper Grande Ronde River trap, 2007 (first time captures). 
Table 6. FL summary by sex for upstream-migrating summer steelhead collected at the upper Grande Ronde River trap, 2007 (first-time captures only).

\begin{tabular}{lcccc}
\hline Sex & Mean FL $(\mathrm{mm})$ & SE & Range & $\mathrm{N}$ \\
\hline Male & 639.4 & 13.6 & $512-792$ & 32 \\
Female & 633.5 & 11.7 & $547-765$ & 33 \\
Total & 636.4 & 8.9 & $512-792$ & 65 \\
\hline
\end{tabular}

Table 7. FL summary by sex for fallback summer steelhead collected at the upper Grande Ronde River trap, 2007.

\begin{tabular}{lcccc}
\hline Sex & Mean FL $(\mathrm{mm})$ & SE & Range & $\mathrm{N}$ \\
\hline Male & 690.0 & 31.3 & $580-787$ & 6 \\
Female & 618.3 & 19.1 & $545-758$ & 12 \\
Total & 642.2 & 17.9 & $545-787$ & 18 \\
\hline
\end{tabular}

Table 8. FL at age summary by age and sex for summer steelhead collected at the upper Grande Ronde River trap, 2007.

\begin{tabular}{lcccccc}
\hline Age & Sex & Mean FL $(\mathrm{mm})$ & SE & Min & Max & $\mathrm{N}$ \\
\hline 1-ocean & $\mathrm{M}$ & 582.1 & 9.3 & 512 & 649 & 15 \\
& $\mathrm{~F}$ & 586.1 & 8.4 & 547 & 637 & 16 \\
& Combined & 584.1 & 6.1 & 512 & 649 & 31 \\
2-ocean & $\mathrm{M}$ & 698.4 & 19.1 & 595 & 792 & 9 \\
& $\mathrm{~F}$ & 686.8 & 13.1 & 598 & 760 & 13 \\
& Combined & 691.6 & 10.8 & 595 & 792 & 22 \\
\hline
\end{tabular}




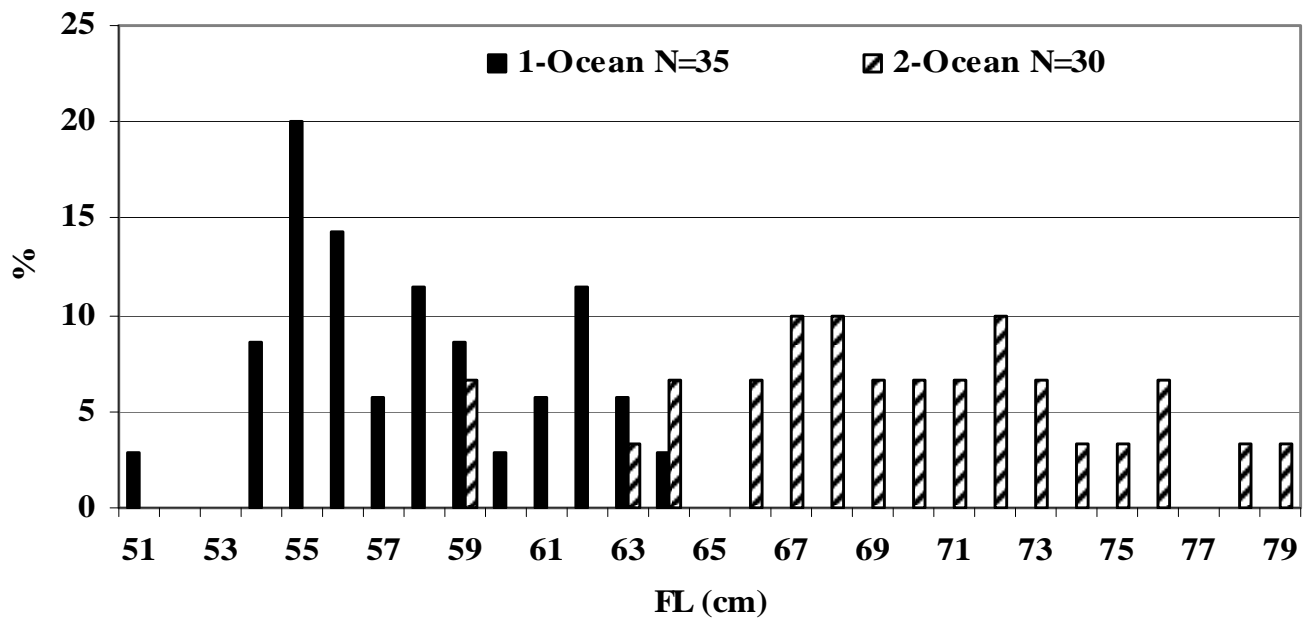

Figure 5. Age-FL (cm) key results for upper Grande Ronde River summer steelhead, 2007.

\section{$2.5 \quad$ Discussion}

The numbers of wild summer steelhead collected at the Catherine Creek trap have been stable over the last 4 years. Trapping conditions have usually been excellent and most of the run each year has been collected. Catches at the upper Grande Ronde River trap have been more variable. There did not appear to be any problems with the weir in 2006 (Ryan Seeger, personal communication), yet no upstream migrants and only two fallbacks were collected. The upper Grande Ronde River trap was moved prior to the 2007 trapping season to a location near Starkey, about 11 mi downstream. Relocation of the upper Grande Ronde River trap may allow collection of a greater number of adults.

Adult life histories for summer steelhead from Catherine Creek and the upper Grande Ronde River are consistent with the general characteristics described for the species by several authors (Burgner et al. 1992, Meehan and Bjornn 1991, Busby et al. 1996, Quinn 2006) and consistent with the limited published data available for other A-run (Busby et al. 1996) populations in the Grande Ronde River basin. Olsen et al. (1992) reported escapement to the larger tributaries in early fall, and smaller tributaries in the spring. Peak migration into Lookingglass Creek occurred in May, with spawning occurring from March through early June, with fry emergence in July and August. Annual estimates of age composition for unmarked adults collected at the Big Canyon trap ranged from 47$69 \%$ 1-salt and 31-53\% 2-salt. Sex ratios (\% females) for unmarked adults collected at the Big Canyon trap ranged from 18-63\% for 1-salt and 2-salt fish. 
Narum et. al. 2006 described genetic variation in the Grande Ronde River, including Catherine Creek and the upper Grande Ronde River. Collections of genetics tissues and archiving at CRITFC will continue. Continuing to collect tissues is prudent and requires little time or expense. Future advances in DNA technology may reduce the cost of analysis and enable scientists to address issues not currently being considered.

\section{$2.6 \quad$ Literature Cited}

Burgner, R. L., J. T. Light, L. Margolis, T. Okazaki, A. Tautz, and S. Ito. 1992. Distribution and origins of steelhead trout (Oncorhynchus mykiss) in offshore waters of the North Pacific Ocean. International North Pacific Fisheries Commission Bulletin Number 51, Vancouver, Canada.

Busby, P. J., T. C. Wainwright, G. J. Bryant, L. J. Lierheimer, R. S. Waples, F. W. Waknitz, and I. V. Lagomarsino. 1996. Status review of west coast steelhead from Washington, Idaho, Oregon, and California. U. S. Department of Commerce, National Oceanic and Atmospheric Administration, Northwest Fisheries Science Center, NOAA Technical Memorandum NMFS-NWFSC 27, Seattle, Washington.

Carmichael, R. W., B. A. Miller, and R. T. Messmer. 1990. Migratory patterns of adult Wallowa stock summer steelhead in the Grande Ronde and Snake Rivers during the 1987-88 run year. Oregon Department of Fish and Wildlife Fish Division Information Report 90-2, Portland, Oregon.

Flesher, M. W., R. W. Carmichael, T. A. Whitesel, and J. R. Ruzycki. 2000. Summer steelhead creel surveys in the Grande Ronde, Wallowa, and Imnaha Rivers for the 19981999 run year. Annual Project Report to the Lower Snake River Compensation Plan, U.

S. Fish and Wildlife Service, Boise, Idaho.

Meehan, W. R. and T. C. Bjornn. 1991. Salmonid distributions and life histories. American Fisheries Society Special Publication 19:47-82.

Narum, S. R., S. Boe, P. Moran, and M. Powell. 2006. Small-scale genetic structure and variation in steelhead of the Grande Ronde River, Oregon, USA. Transactions of the American Fisheries Society 135:979-986.

National Marine Fisheries Service (NMFS). 1999. Biological Opinion on Artificial Propagation In The Columbia River Basin, Portland, Oregon.

Olsen, E., P. Pierce, M. McLean, and K. Hatch. 1992. Stock summary report for Columbia River anadromous salmonids. Volume II. Oregon subbasins above Bonneville 
Dam for the coordinated information system. Report to the Bonneville Power Administration, Project 198810800, Portland, Oregon.

Quinn, T. P. 2006. The behavior and ecology of Pacific salmon and trout. University of Washington Press, Seattle.

Ricker, W. E. 1975. Computation and interpretation of biological statistics of fish populations. Bulletin 191 of the Fisheries Research Board of Canada, Department of the Environment, Fisheries and Marine Service, Ottawa.

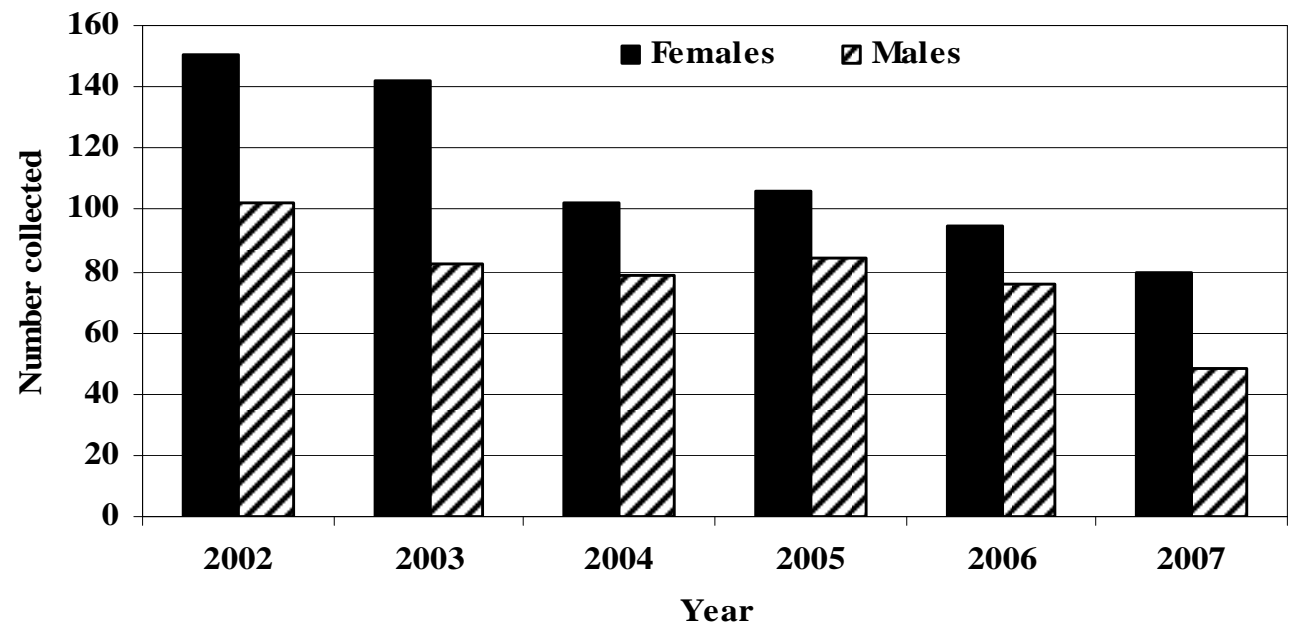

Appendix Figure 1. Upstream-migrating wild summer steelhead collected by sex at the Catherine Creek trap, 2002-2007. 


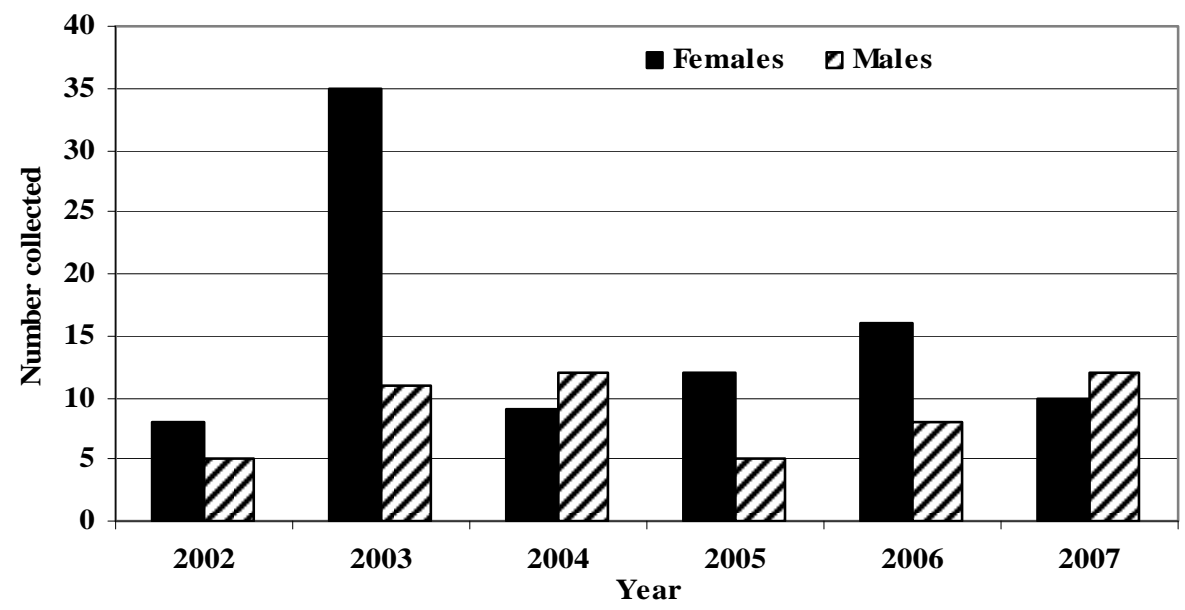

Appendix Figure 2. Fallback wild summer steelhead collected by sex at the Catherine Creek trap, 2002-2007.

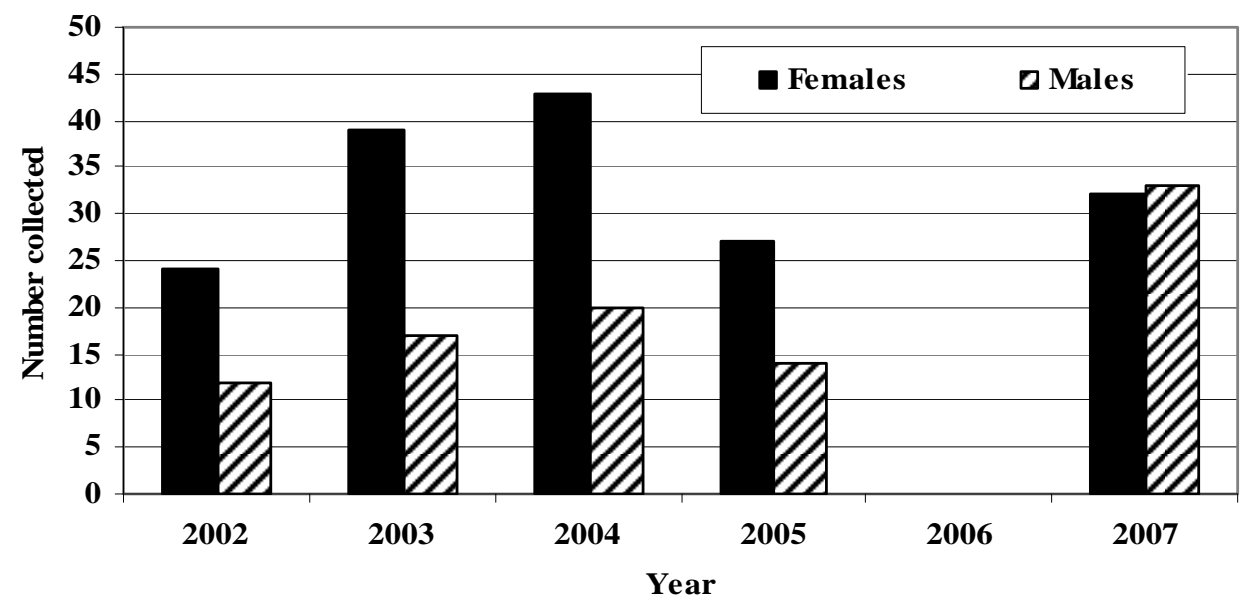

Appendix Figure 3. Upstream-migrating wild summer steelhead collected by sex at the upper Grande Ronde River trap, 2002-2007. 


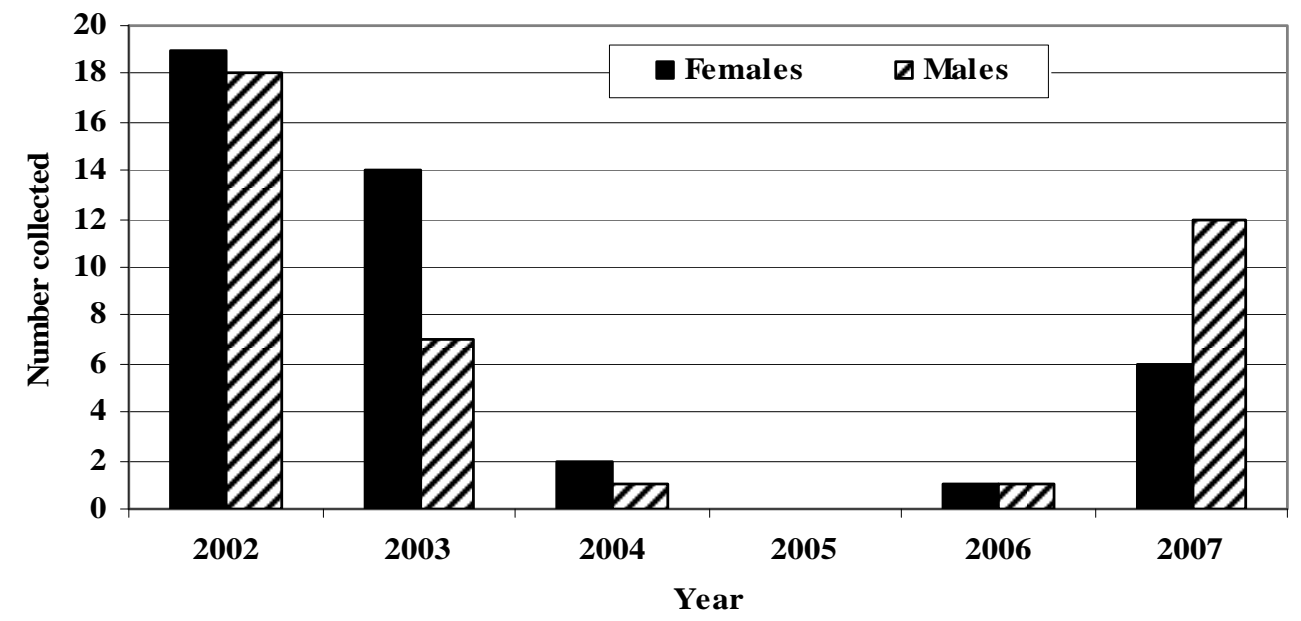

Appendix Figure 4. Fallback wild summer steelhead collected by sex at the upper Grande Ronde River trap, 2002-2007.

\section{SECTION III. ASSISTANCE TO PROGRAM COOPERATORS}

Program staff assisted in conducting spawning ground surveys in the Grande Ronde River basin, the Imnaha River, Wenaha River and John Day River. Staff also assisted in spawning of Imnaha River spring Chinook salmon, collecting captive brood parr from Catherine Creek and the upper Grande Ronde River and spawning of captive broodstock Chinook salmon at Bonneville Fish Hatchery.

\section{ACKNOWLEDGEMENTS}

Brian Jonasson, Pat Keniry, and Fred Monzyk (ODFW) provided unpublished early life history, spawning ground survey, and juvenile pretransfer data. Debbie Eddy (ODFW) provided conventional broodstock data and Diane Deal (ODFW) fecundity data. Mike McLean and his crew collected data and tissues at the adult collection and juvenile acclimation sites. Gary James, Michelle Thompson, and Julie Burke, and Celeste Reves (CTUIR) provided program administrative assistance. Teena Ballard and Jennifer Wiseman-Yancey (USFS) provided water temperature data. 